\title{
Towards Uncovering the Role of Pre-fibrillar Oligomers of $\alpha$-Synuclein in the Pathogenesis of Parkinson's Disease
}

\section{PhD Thesis}

\author{
in partial fulfillment of the requirements \\ for the degree of "Doctor of Philosophy (PhD)" \\ in the Molecular Biology Program \\ at the Georg August University Goettingen, \\ Faculty of Biology
}

\author{
submitted by \\ Madhu Babu Gajula Balija
}

born in

Kodumur, India

April 2010 
I hereby declare that the $\mathrm{PhD}$ thesis entitled, "Towards Uncovering the Role of Pre-fibrillar Oligomers of $\alpha$-Synuclein in the Pathogenesis of Parkinson's Disease," has been written independently and with no other sources and aids than quoted.

Madhu Babu Gajula Balija 


\section{Dedicated to}

My wonderful parents...

for their efforts to set me on my path.

for showing me the real value of education in life by themselves having fated to live practically without any formal education.

\section{My remarkable teachers...}

for instilling the right approach towards knowledge and learning.

for their efforts to infuse right values into life.

The IMPRS Molecular Biology/the Max Planck Institute...

for nurturing my passion for science.

for the transformational learning experience. 


\section{CONTENTS}

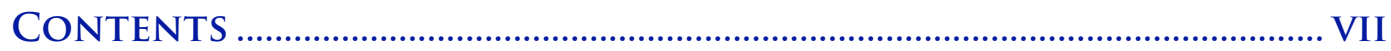

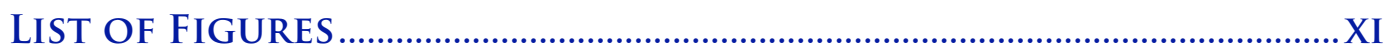

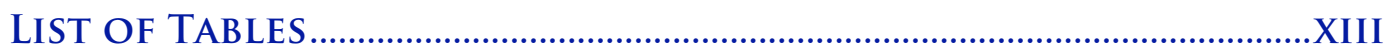

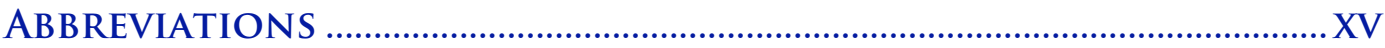

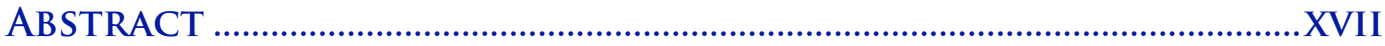

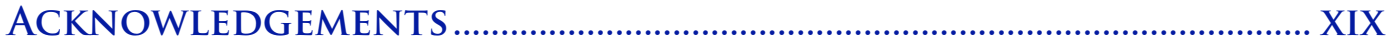

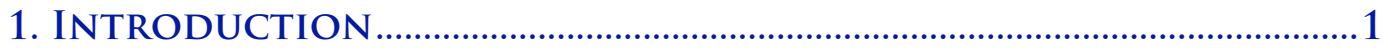

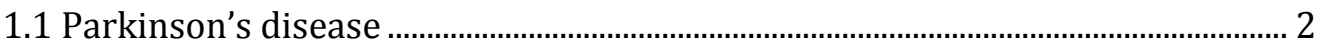

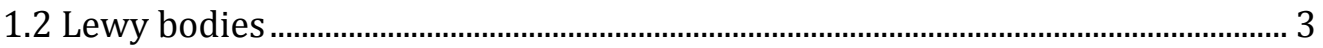

$1.3 \alpha$-Synuclein structure and function............................................................................. 4

1.4 Misfolding and aggregation of $\alpha$-Synuclein ............................................................. 6

1.5 Soluble pre-fibrillar oligomers in neurodegeneration-revisiting the amyloid

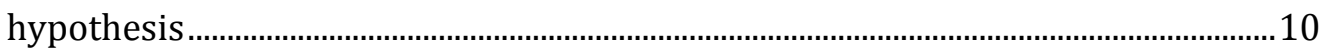

1.6 Pathogenicity of pre-fibrillar soluble oligomers......................................................12

1.7 Drosophila model for Parkinson's disease.................................................................. 14

1.8 Understanding motor and non-motor symptoms of PD in the Drosophila

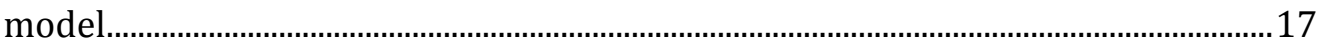

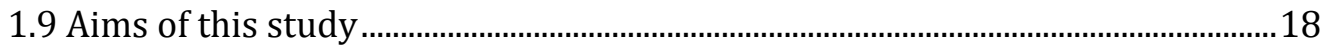

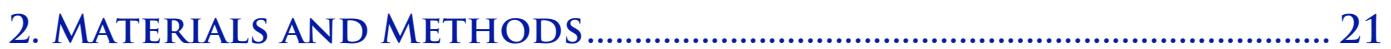

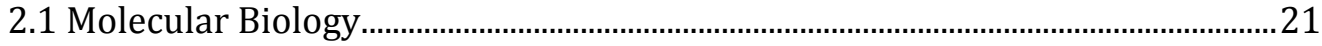

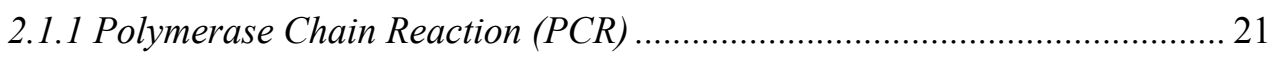

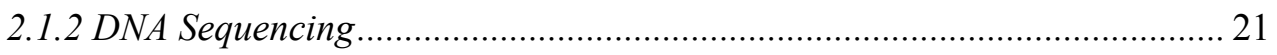

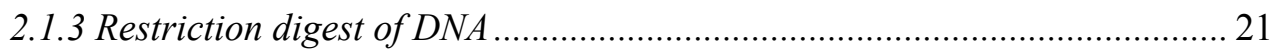

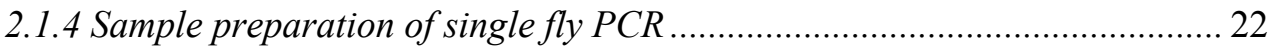




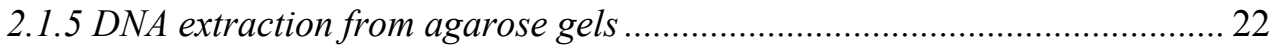

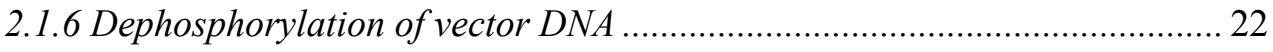

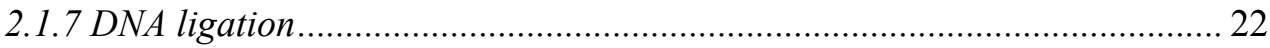

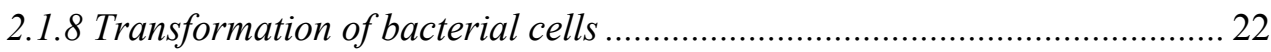

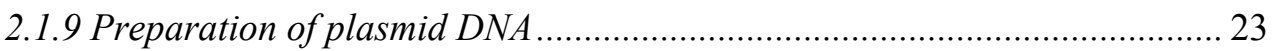

2.1.10 DNA preparation for embryo injections ................................................... 23

2.1.11 Preparation of fly head extracts ............................................................ 23

2.1.12 Polyacrylamide Gel Electrophoresis (PAGE) ........................................... 24

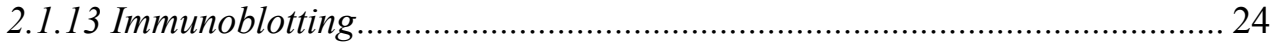

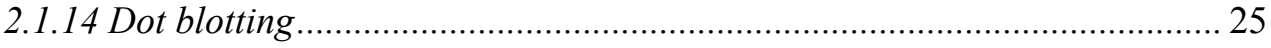

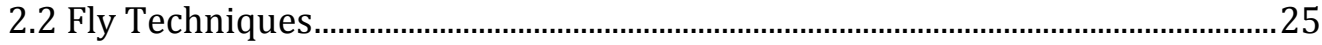

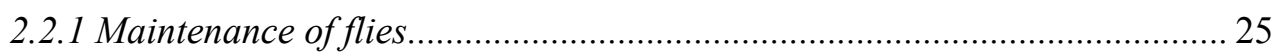

2.2.2 Generation of stable transgenic fly lines ...................................................... 25

2.2.3 Ectopic gene expression via the UAS/GAL4 system ..................................... 26

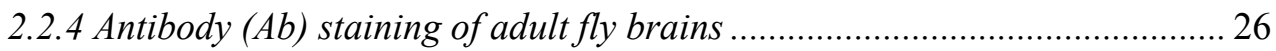

2.2.5 Dissection of larval NMJs (Neuromuscular Junctions) ............................. 27

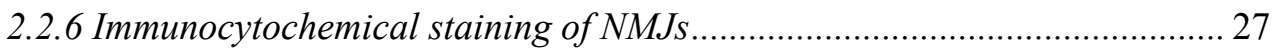

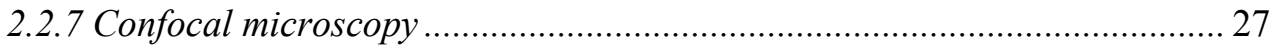

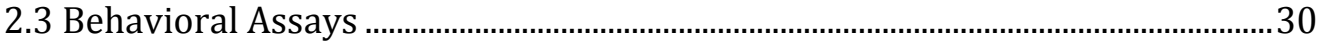

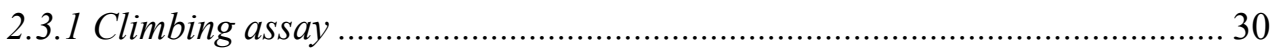

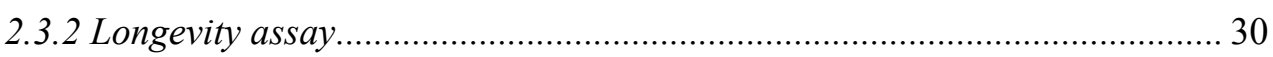

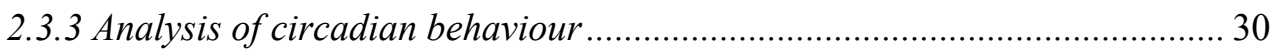

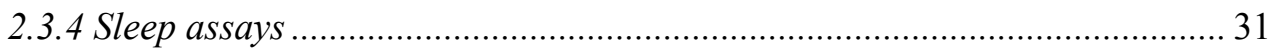

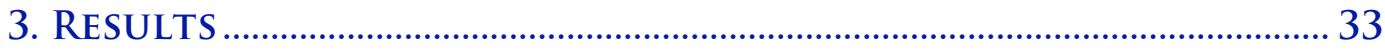

3.1 Structure-based rational design of the $\alpha$-synuclein variants.

3.2 TP- $\alpha$ S and A56P- $\alpha$ S show increased propensity to form oligomers and

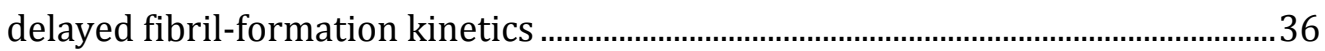

3.3 Generating the Drosophila model for Parkinson's disease...................................38

3.4 Pre-fibrillar oligomer-enriched structural variants of $\alpha \mathrm{S}$ cause motor deficits 40

3.5 Pre-fibrillar oligomer-enriched $\alpha$ S structural variants reduce longevity .......42

3.6 Expression of pre-fibrillar structural $\alpha \mathrm{S}$ variants causes age-dependent degeneration of dopaminergic (DA) neurons 
3.6.1 No degeneration of DA neurons in young flies expressing $\alpha S$ variants....... 44

3.6.2 Degeneration of DA neurons in old flies expressing $\alpha S$ variants ................ 46

3.7 Dopamine dependent behavioral deficits in flies expressing $\alpha \mathrm{S}$ variants........48

3.8 Sleep abnormalities in PD flies expressing $\alpha$ S variants 48

3.9 Abnormalities in circadian locomotor activity behavior of flies expressing an oligomer-forming $\alpha \mathrm{S}$ variant. 52

3.10 Circadian defects in response to TP- $\alpha$ S expression can be mimicked by DA neuron inactivation

3.11 Defective localization of neurotoxic $\alpha \mathrm{S}$ variants to the synaptic terminals in vivo

4. DISCUSSION

4.1 Accumulation of $\alpha$ S has a toxic gain-of function.

4.2 Soluble oligomers of $\alpha$-synuclein are the likely culprits in PD pathogenesis 68

4.3 Formation of more pre-fibrillar oligomers by the TP- $\alpha$ S and A56P- $\alpha S$ variants

4.4 An improved Drosophila PD model to study the pathogenicity of oligomerforming $\alpha$ variants

4.5 Motor deficits and reduced longevity: human PD-like features in the flies expressing pre-fibrillar oligomer-forming $\alpha S$ variants. .75

4.6 Degeneration of DA neurons in the flies expressing pre-fibrillar oligomerforming $\alpha S$ variants

4.7 Non-motor deficits in PD flies expressing pre-fibrillar oligomer forming $\alpha S$ variants .77

4.8 Sleep abnormalities in the PD flies . 78

4.9 Circadian dysfunction in the flies expressing most toxic oligomer-forming TP- $\alpha \mathrm{S}$ 80

4.10 Synucleinopathies are synaptopathies: defective localization of the neurotoxic $\alpha S$ variants at the synaptic terminals of larval NMJs 


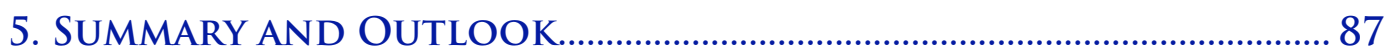

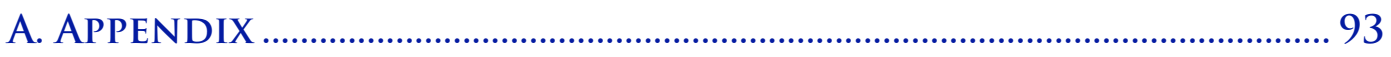

Algorithms used for the sleep analysis in Drosophila …................................................93

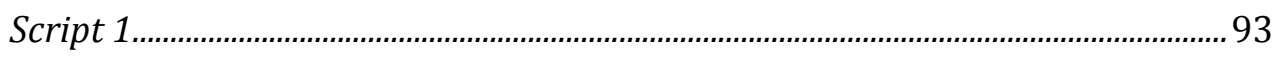

Script 2

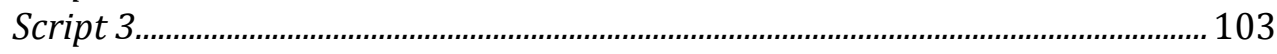

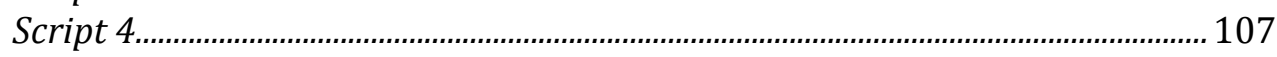

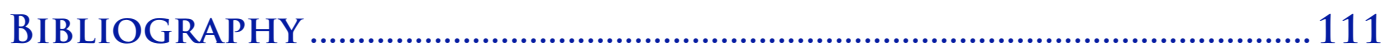

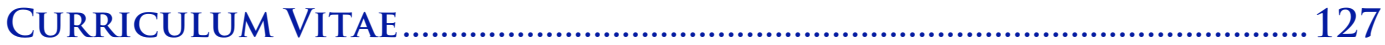




\section{LIST OF FIGURES}

Figure 1: Lewy bodies in the human PD brain contain amyloid fibrils of $\alpha S$ 3

Figure 2: Domain comparisons of the Synuclein protein family 5

Figure 3: Domain structure of the $\alpha S$ and human PD related missense mutations 6

Figure 4: Amyloid aggregates of misfolded proteins in the neurodegenerative diseases 7

Figure 5: Schematic representation of the general mechanism of aggregate formation _ 9

Figure 6: Factors promoting the formation of pre-fibrillar oligomers may cause earlyonset of the disease

Figure 7: Schematic depiction of the potential pathogenic mechanisms in $P D$

Figure 8: $\alpha$-Synuclein variants with reduced fibrillation propensity and impaired $\beta$ structure form more on-pathway pre-fibrillar oligomers

Figure 9: Illustration of the summary of results based on in vitro experiments with structure based design variants of the $\alpha S$

Figure 10: Generating the Drosophila model for Parkinson's disease by using $\phi-C 31$ based site-specific recombination method

Figure 11: Drosophila expressing pre-fibrillar oligomer enriched $\alpha$-Synuclein variants show motor deficits in the climbing assay

Figure 12: Longevity of flies expressing different variants of $\alpha S$ and LacZ 43

Figure 13: Dopaminergic (DA) neurons in the young flies expressing different variants of $\alpha S$ and LacZ 45

Figure 14: Age dependent degeneration of DA neurons in the flies expressing different variants of $\alpha S$ 46

Figure 15: Flow chart depicting the sleep assay and analysis in Drosophila 49 
Figure 16: Dopamine dependent sleep deficits in the flies expressing different variants of $\alpha S$

Figure 17: Schematic representation of a typical circadian experiment 53

Figure 18: Properties of rhythmic data 55

Figure 19: Circadian locomotor activity profiles of young Drosophila expressing WT- $\alpha S$ and $T P-\alpha S$ 56

Figure 20: Circadian locomotor activity profiles of old Drosophila expressing WT- $\alpha S$ and $T P-\alpha S$ 57

Figure 21: Age dependent circadian defects in Drosophila expressing TP- $\alpha S$ in the DA neurons

Figure 22: Schematic representation of inactivation/attenuation of the Dopaminergic (DA) neurons

Figure 23: Circadian defects caused by TP- $\alpha S$ mimic the effects of DA neuron inactivation by using genetic methods 62

Figure 24: Defective localization of $\alpha S$ variants to the synaptic terminals 65

Figure 25: Caricatured representation of the conclusive summary 85

Figure 26: Circadian locomotor activity profiles of old Drosophila expressing WT- $\alpha S$ and $A 53 T-\alpha S$ 108 


\section{LIST OF TABLES}

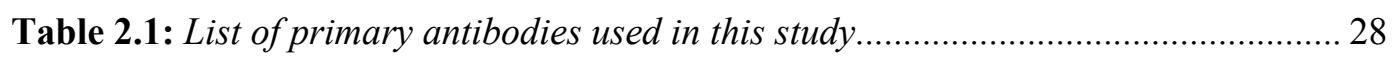

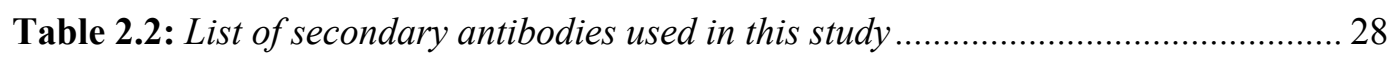

Table 2.3: List of fly stocks used and generated in this study ...................................... 29 


\section{ABBREVIATIONS}

$\mathrm{Ab}$

AD

ALS

ANOVA

$a t t B$

attP

AWK

$\mathrm{A} \beta$

BRP

cAMP

CNS

DA

DA neurons

DAM

DD

Ddc-Gal4

DL

DM

DNA

EGFR

EJC

EKO

ER

$\mathrm{hr}$

HRP
Antibody

Alzheimer's Disease

Amyotrophic Lateral Sclerosis

Analysis of Variance

Attachment site B

Attachment site $\mathrm{P}$

Programming language called AWK

Amyloid- $\beta$

Bruchpilot

cyclic Adenosine Monophosphate

Central Nervous System

Dopamine

Dopaminergic neurons

Drosophila Activity Monitors

Dark-Dark

Dopamine decarboxylase-Gal4

Dorsolateral

Dorsomedial

Deoxyribonucleic Acid

Epidermal Growth Factor Receptor

Excitatory Junctional Currents

Electrical Knockout

Endoplasmic Reticulum

Hour

Horse Radish Peroxidase 


\begin{tabular}{|c|c|}
\hline lacZ & Gene that encodes $\beta$-D-galactosidase \\
\hline LB & Luria Bertani broth \\
\hline LBs & Lewy Bodies \\
\hline LD & Light-Dark \\
\hline LED & Light Emitting Diode \\
\hline MB & Mushroom Body \\
\hline mEJC & miniature Excitatory Junctional Currents \\
\hline NAC & Non-A $\beta$ Component \\
\hline $\mathrm{NaChBac}$ & Bacterial Sodium Channel \\
\hline NMJ & Neuromuscular Junctions \\
\hline PAGE & Polyacrylamide Gel Electrophoresis \\
\hline PBS & Phosphate Buffered Saline \\
\hline PCR & Polymerase Chain Reaction \\
\hline PD & Parkinson's Disease \\
\hline Phi-C31 & Bacteriophage Phi-C31 \\
\hline PPR & Paired Pulse Ratio \\
\hline SDS & Sodiumdodecyl Sulfate \\
\hline SEM & Standard Error Mean \\
\hline $\mathrm{SNpc}$ & Substantia Nigra pars compacta \\
\hline SUV & Small Unilamellar Vesicle \\
\hline $\mathrm{TH}$ & Tyrosine Hydroxylase \\
\hline TH-Gal4 & Tyrosine Hydroxylase-Gal4 \\
\hline ThT & Thioflavin $\mathrm{T}$ \\
\hline TP- $\alpha \mathrm{S}$ & A30P/A56P/A76P/ $\alpha-$ Synuclein \\
\hline UAS & Upstream Activating Sequence \\
\hline UPR & Unfolded Protein Response \\
\hline WT- $\alpha \mathrm{S}$ & Wildtype- $\alpha$-Synuclein (human) \\
\hline$\alpha S$ & $\alpha$-Synuclein \\
\hline$\mu \mathrm{g}$ & Microgram \\
\hline$\mu 1$ & Microlitre \\
\hline
\end{tabular}




\section{ABSTRACT}

Parkinson's disease (PD) is a progressive neuropathological disorder that is characterized by the presence of intra-cytoplasmic inclusions called Lewy Bodies (LBs). LBs represent insoluble protein aggregates with $\alpha$-Synuclein $(\alpha \mathrm{S})$ as a major component. LBs also mark lesions found in a range of related disorders, collectively referred to as synucleinopathies. Recent studies suggest that pathogenesis could result from small pre-fibrillar intermediates of the aggregation pathway, i.e. soluble oligomers of misfolded $\alpha$-Synuclein. So, it is not decided yet whether the soluble oligomers or the final products of the $\alpha \mathrm{S}$ aggregation (i.e. the $\alpha S$ fibrils) are cause of the disease, meaning that the nature of pathogenic $\alpha S$ species is still unresolved.

To understand the nature of pathogenic $\alpha \mathrm{S}$ species and to investigate whether accumulation of soluble pre-fibrillar oligomers of $\alpha \mathrm{S}$ is responsible for the increased neurotoxicity in PD, I have used rationally designed structural variants of $\alpha \mathrm{S}$ with different aggregation properties and tested their biological properties in Drosophila melanogaster, which lacks an $\alpha \mathrm{S}$ gene in the wild type genome. Two of the designed variants, TP- $\alpha \mathrm{S}$ and A56P- $\alpha \mathrm{S}$, have a remarkably decreased propensity to aggregate, and they form more pre-fibrillar soluble oligomeric species in aggregation assays in vitro. In order to examine their biological effects in vivo, I have employed Drosophila as a tool to explore possible PD-like effects of the $\alpha \mathrm{S}$ mutants. The $\alpha \mathrm{S}$ mutant genes were inserted into the Drosophila genome. To avoid possible "position effects" affecting the expression of the individual transgenes, I used the phiC31 site-specific integration system. The site specific genomic integration of the $\alpha \mathrm{S}$ gene and the different $\alpha \mathrm{S}$ mutants permit to directly compare the effects of the different $\alpha \mathrm{S}$ variants on 
various biological parameters such as longevity, climbing (negative geotactic and phototactic responses), sleep behavior and circadian rhythmicity that were used as sensitive readouts for neurotoxicity in response to $\alpha S$ activity expressed specifically in the Dopaminergic (DA) neurons. The results show that the TP- $\alpha \mathrm{S}$ and A56P- $\alpha \mathrm{S}$ mutants caused increased neurotoxicity as compared to wild type $\alpha \mathrm{S}$ or expression of the bacterial lacZ gene serving as controls. Immunohistochemistry on adult fly brains confirm that neurotoxicity as revealed by the behavior tests correlates well with the degeneration of DA neurons in response to the expression of the $\alpha \mathrm{S}$ variants. The data show that the pre-fibrillar oligomer-forming TP- $\alpha \mathrm{S}$ mutant is most toxic among the $\alpha \mathrm{S}$ variants. The results are consistent with the conclusion that pre-fibrillar oligomers of $\alpha \mathrm{S}$ are highly toxic pathogenic species in the neurodegeneration process associated with PD. Aside from supporting the hypothesis that the pre-fibrillar status of $\alpha \mathrm{S}$ is already toxic for neurons, the Drosophila system presented here delivers a novel experimental system in which the non-motor aspects of $\mathrm{PD}$, the mechanism of cellular action and likely even the efficacy of lead compounds leading to therapeutic drugs can be addressed. 


\section{ACKNOWLEDGEMENTS}

First of all, I am highly indebted to Prof. Dr. Herbert Jäckle and Prof. Dr. Christian Griesinger for giving me the great opportunity to carry out this work, for their scientific and personal support, for the intellectual freedom bestowed upon me, and for being the ultimate source of inspiration.

I am grateful to Prof. Dr. Markus Zweckstetter and Dr. Alf Herzig for the stimulating scientific discussions and their interest in my work. My special thanks goes to Dr. Stefan Eimer and Prof. Dr. Stephan Sigrist for their time both as members of my $\mathrm{PhD}$ thesis committee and as wonderful collaborators.

This work would not have been fun and easy without the cheerful ambience and timely help I received from my colleagues and collaborators in the departments of Molecular Developmental Biology and NMR based Structural Biology at the MPI for biophysical chemistry. I specially wish to thank Pinar Karpinar for her cooperation in this project and for being a nice classmate in IMPRS program.

My dearest appreciation goes to Ana Martinez, Matthias Samwer, Matias Hernandez, Christian Kappel, Veronica Mueller, and other members of the $\mathrm{PhD}$ Students Committee' at MPIbpc for giving me the joy of sharing and working collectively to enrich social and scientific life of others at the institute.

Finally, I would like to express my deepest sense of gratitude to all the friends and family members who stood beside me and extended their help during the vicissitudes of life in all these years: Suresh Koduru, Sai Kumar, Sreehari, Patrick Mueller, George, Pitchaiah Cherukuri, Aniket, Mayur, Ashutosh, and Jagadeesh. 


\section{INTRODUCTION}

Brain is a highly complex organ made up of extremely intricate and enigmatic neuronal circuits that govern crucial functions of the organism. Integrity and functions of the neuronal circuits are controlled by the health and wellbeing of neurons that constitute them. The neuronal cellular machineries are often subjected to malfunction and dysregulation because of the genetic and sporadic perturbations that will eventually lead to neurodegeneration. Progressive neuronal loss and neurological impairment are hallmarks of the neurodegenerative disorders. Prevalence of neurodegenerative disorders is especially high in the countries with more life expectancy and demographically aging population, as age is one of the risk factor associated with neurodegeneration (Forman et al., 2004; Outeiro et al., 2007). A wide variety of neurodegenerative disorders are clinically and neuropathologically characterized and they are linked to the formation of intracellular and extracellular fibrillar aggregates of misfolded proteins. Such diseases include Alzheimer's, Parkinson's, Huntington's, and dementia with Lewy bodies, Spinocerebellar ataxia, and Multiple systems atrophy etc.) (Chiti and Dobson, 2006). Here, I focus mainly on the $\alpha$-Synuclein $(\alpha S)$ aggregation and its relation to pathogenesis in Parkinson's disease (PD) as well as other synucleinopathies. 


\subsection{Parkinson's disease}

$\mathrm{PD}$ is the second most common neurodegenerative disease in Europe with an incidence of more than one patient per thousand inhabitants. People are diagnosed usually in their 40ies, but disease is more prevalent with the more than 60 years old people with no difference in gender (http://www.europarkinson.net).

Nearly 200 years ago, James Parkinson initially described PD as 'shaking palsy' in his monograph An Essay on the Shaking Palsy (see Factor, 2002). Later, in recognition of this remarkable work, the French neurologist Charcot referred to 'shaking palsy' as Parkinson's disease. However, even long before these descriptions, there were accounts of a clinical syndrome consisting of tremor and akinesia described in Indian medical (Ayurvedic) texts written more than 3000 years ago (reviewed in Katzenschlager et al., 2004; Manyam, 1990). This syndrome, termed Kampavata in Sanskrit, was treated with natural products from Mucuna pruriens, a plant later now known to contain levodopa (L-dopa). Incidentally, L-dopa is a drug that was used from the mid- $20^{\text {th }}$ century onwards for the treatment of PD symptoms and its use has revolutionized the PD therapy (Lee and Trojanowski, 2006).

There has been an unprecedented development in understanding of the Parkinson's and other neurodegenerative disorders in the past two decades (reviewed in Lee and Trojanowski, 2006) . By now, important clinical features of the PD include chronic and progressive neurodegeneration characterized by severe motor symptoms such as bradykinesia (slowness in initiating voluntary movements), resting tremor, rigidity (increased resistance to passive movement) and postural instability (balance difficulties). In addition, many non-motor symptoms like sleep-disorders, autonomic disturbances, cognitive dysfunction and depression are common among the PD patients. In neuropathological terms, the key defining features of PD are the loss of the dopaminergic (DA) neurons in the Substantia Nigra pars Compacta $(\mathrm{SNpc})$ and the presence of intracytoplasmic inclusions called Lewy bodies (LBs) or Lewy body containing neurites, termed Lewy neurites (Factor, 2002; Moore et al., 2005; Shults, 2006). 


\subsection{Lewy bodies}

LBs are the common neuropathological lesions found in PD and a group of related neurodegenerative disorders called synucleinopathies (Figure 1). LBs found in synucleinopathies such as PD, dementia with Lewy bodies, and Multiple systems atrophy as well as a number of other neurodegenerative disorders contain the insoluble fibrillar aggregates of $\alpha \mathrm{S}$ (reviewed in Shults, 2006; Spillantini et al., 1997). $\alpha \mathrm{S}$ is the major protein component of LBs (Shults, 2006), which is an abundant neuronal protein expressed in the central nervous system (CNS). Though LBs are an idiosyncratic feature of the synucleinopathies including autosomal dominant familial PD, there are very few exceptional neurodegenerative diseases which don't show the formation of LBs. Few examples in this category are: Autosomal recessive juvenile parkinsonism (ARJP, a juvenile-onset form of PD) (Kitada et al., 1998), Gerstmann-Straussler syndrome (a genetic prion disease; (Hsiao and Prusiner, 1991) and sporadic ALS (Amyotrophic lateral sclerosis) (Rosen, 1978).

With the growing list of neurodegenerative diseases, experimental evidence drawn primarily from the disciplines of neuropathology, genetics and biophysics strongly supports hypothesis that protein aggregation triggers the cascade of events that finally result in neurodegeneration and disease (Hardy and Selkoe, 2002).

\section{(on the next page)}

Figure 1: Lewy bodies in the human PD brain contain amyloid fibrils of $\alpha \mathrm{S}$

Human brain (left panel) picture showing the regions of brain involved in Dopamine signaling and affected in PD. Lewy body (intracytoplasmic neuronal inclusions) found in the human PD brains (middle panel)(Spillantini et al., 1997). Electron micrographs of the amyloid fibrils of $\alpha \mathrm{S}$ (right panel) found in Lewy bodies and in vitro preparations (Goedert, 2001). 


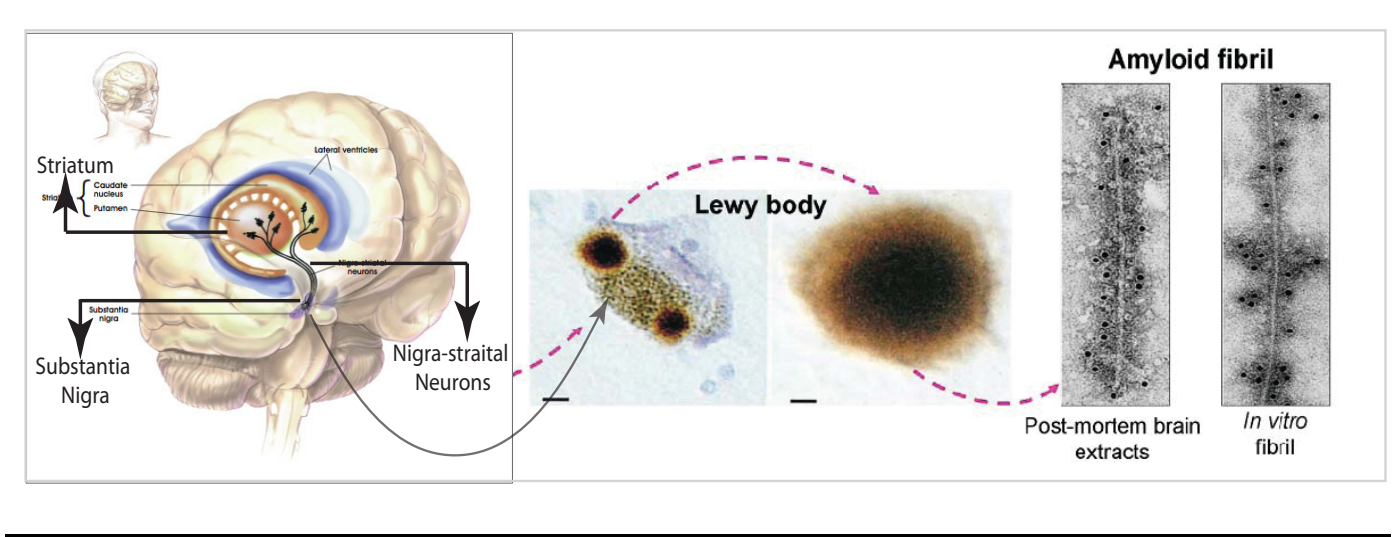

\section{$1.3 \alpha$-Synuclein structure and function}

$\alpha$-Synuclein is an abundantly expressed protein in the CNS (Iwai et al., 1995). It belongs to a highly conserved family of proteins consisting of $\alpha-, \beta-$, and $\gamma$ synuclein (Figure 2) (Clayton and George, 1998). $\alpha \mathrm{S}$ consists of 140 amino acid residues and is the longest synuclein polypeptide. It is encoded by the five of the seven exon containing $\alpha \mathrm{S}$ gene (Goedert, 2001). $\alpha \mathrm{S}$ was first cloned from Torpedo californicum (Maroteaux et al., 1988) and later identified in humans as the non-A $\beta$ component (NAC) of Alzheimer's disease amyloid precursor NACP (Ueda et al., 1993). $\alpha \mathrm{S}$ is a major component of the LBs.

In aqueous solution, monomeric $\alpha \mathrm{S}$ is highly flexible and belongs to the class of intrinsically disordered proteins, with transient long-range interactions stabilizing a closed conformation (Bertoncini et al., 2005; Dedmon et al., 2005). Structurally, the $\alpha \mathrm{S}$ protein is divided into three parts: (i) amphiphatic N-terminal region, (ii) the non- $\mathrm{A} \beta$ component (NAC) region and (iii) the acidic $\mathrm{C}$-terminal region (Figure 2). The N-terminal region is composed almost entirely of six variants of a degenerate 11-amino acid consensus motif with slight variations of the sequence KTKEGV (Figure 3). The 11-mer repeats comprise an apolipoprotein-like-A2 helix, which mediates binding to phospholipids vesicles. These 11-mer repeats are highly conserved among the $\alpha$-, $\beta$-, and $\gamma$-synuclein. The central regions of the synucleins contain two of the 11-mer repeats and the hydrophobic NAC portion spanning 71-82 amino acid residues. The NAC region has a crucial role in fibrillation and thus, $\alpha \mathrm{S}$ lacking this region doesn't fibrillate 
(Shults, 2006). The C-terminus of $\alpha \mathrm{S}$ is not conserved among the synucleins. It has the preponderance of acidic amino acid residues and is subjected to many posttranslational modifications (Goedert, 2001).

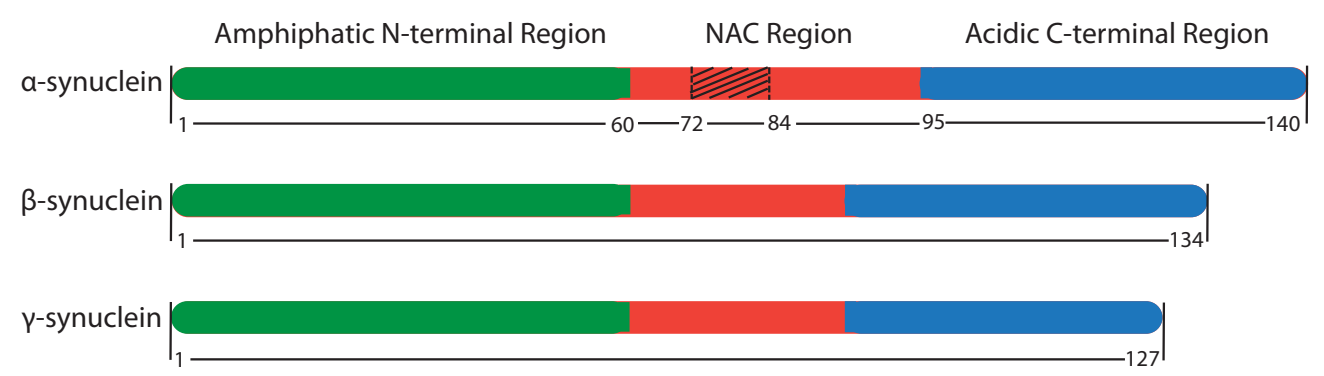

Figure 2: Domain comparisons of the Synuclein protein family

Primary sequences of the $\alpha-, \beta-, \gamma$-synucleins are highly conserved. Their sequences can be divided into three regions. Highly conserved amphiphatic N-terminal region (green), hydrophobic NAC region (red), and less conserved acidic C-terminal region (blue). The extra eleven amino acid residues present in the NAC region of $\alpha$-synuclein (hatched box within the red region) bestow it the unique property of aggregation among its family members.

The precise cellular function of the $\alpha \mathrm{S}$ is still enigmatic. However, many studies implicate a potential role of $\alpha S$ in synaptic function. $\alpha \mathrm{S}$ is a presynaptic protein (George et al., 1995; Iwai et al., 1995) that is highly expressed in the central nervous system. $\alpha \mathrm{S}$ is enriched in synaptosomal preparations, but not found in purified fractions containing the synaptic vesicles (Kahle et al., 2000). These findings support a possible role of $\alpha \mathrm{S}$ in the synaptic function and hints that it might be a visiting protein of the synaptic vesicles (Takamori et al., 2006). $\alpha \mathrm{S}$ is not necessary for the normal development as $\alpha \mathrm{S}$ knockout mice were viable and fertile, and had normal brain architecture, particularly of the nigrostriatal DA system (Abeliovich et al., 2000). Nevertheless, $\alpha \mathrm{S}$ is shown to have a cooperative function together with the cystein-string-protease- $\alpha(\mathrm{CSP} \alpha)$ in preventing neurodegeneration (Chandra et al., 2005), and some regulatory function on the reserve or resting pool of presynaptic vesicles (Cabin et al., 2002; Chandra et al., 
2004). Lack of known defined function in a specific biological pathway makes it formidably difficult to understand the role of $\alpha S$ in pathophysiology and neurodegeneration of Parkinson's disease and other synucleinopathies.

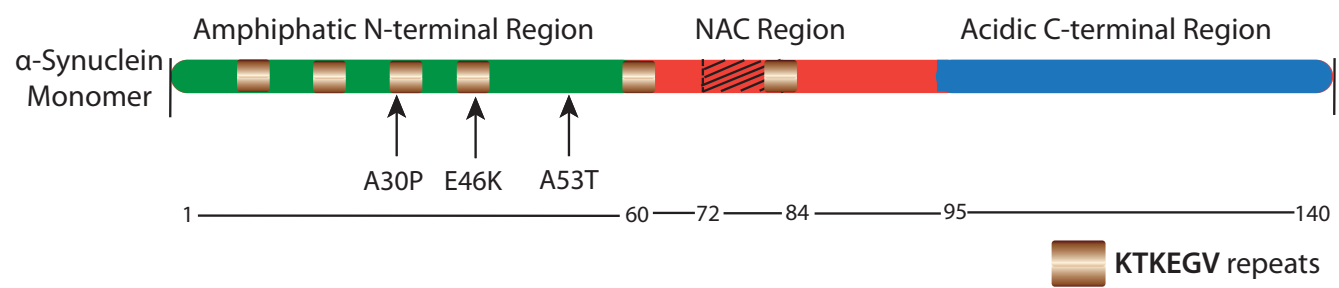

Figure 3: Domain structure of the $\alpha \mathrm{S}$ and human PD related missense mutations

The N-terminal region of $\alpha \mathrm{S}$ has imperfect 11-mer repeats with KTKEGV hexamer motif. The acidic C-terminal region (blue) is susceptible to many posttranslational modifications and it is known to inhibit aggregation. Point mutations in the $\alpha \mathrm{S}, \mathrm{A} 30 \mathrm{P}-\alpha \mathrm{S}$, E46K- $\alpha$ S, and A53T- $\alpha$ S (black arrows) are known to cause dominant familial PD in humans.

\subsection{Misfolding and aggregation of $\alpha$-Synuclein}

Proteins are dynamic entities continuously engaged in the process of folding and unfolding/partial unfolding in response to the demands set by cellular environments. In doing so, proteins tend to misfold and adopt alternative conformations that are completely non-physiological (Dobson, 2003). Natively unfolded proteins like $\alpha \mathrm{S}$ have a high tendency to misfold. If cellular surveillance mechanisms are overridden by the factors that promote misfolding, it will either result in proteins with compromised function or aggregated proteins (Chiti and Dobson, 2006; Selkoe, 2003). Aggregates are formed when proteins with misfolded conformations come together and stabilize themselves with more intermolecular interactions (Figure 4 shows some aggregates formed by the misfolded proteins in different neurodegenerative disorders). Aggregation is favored because the energy minima of aggregates/aggregation-intermediates resulting from inter-molecular interactions between misfolded conformations are less than that of the native protein stabilized by intra-molecular interactions 
(Dobson, 2003). The misfolded conformations in aggregation pathway may further seed or nucleate the process of aggregation, thereby promoting the formation of more pre-fibrillar intermediates, which finally result in the neurological and systemic diseases (reviewed in Lashuel and Lansbury, 2006; Selkoe, 2003; Soto, 2003). For this reason, the neurodegenerative diseases like Parkinson's, Alzheimer's, and Huntington's are often called protein conformational disorders.

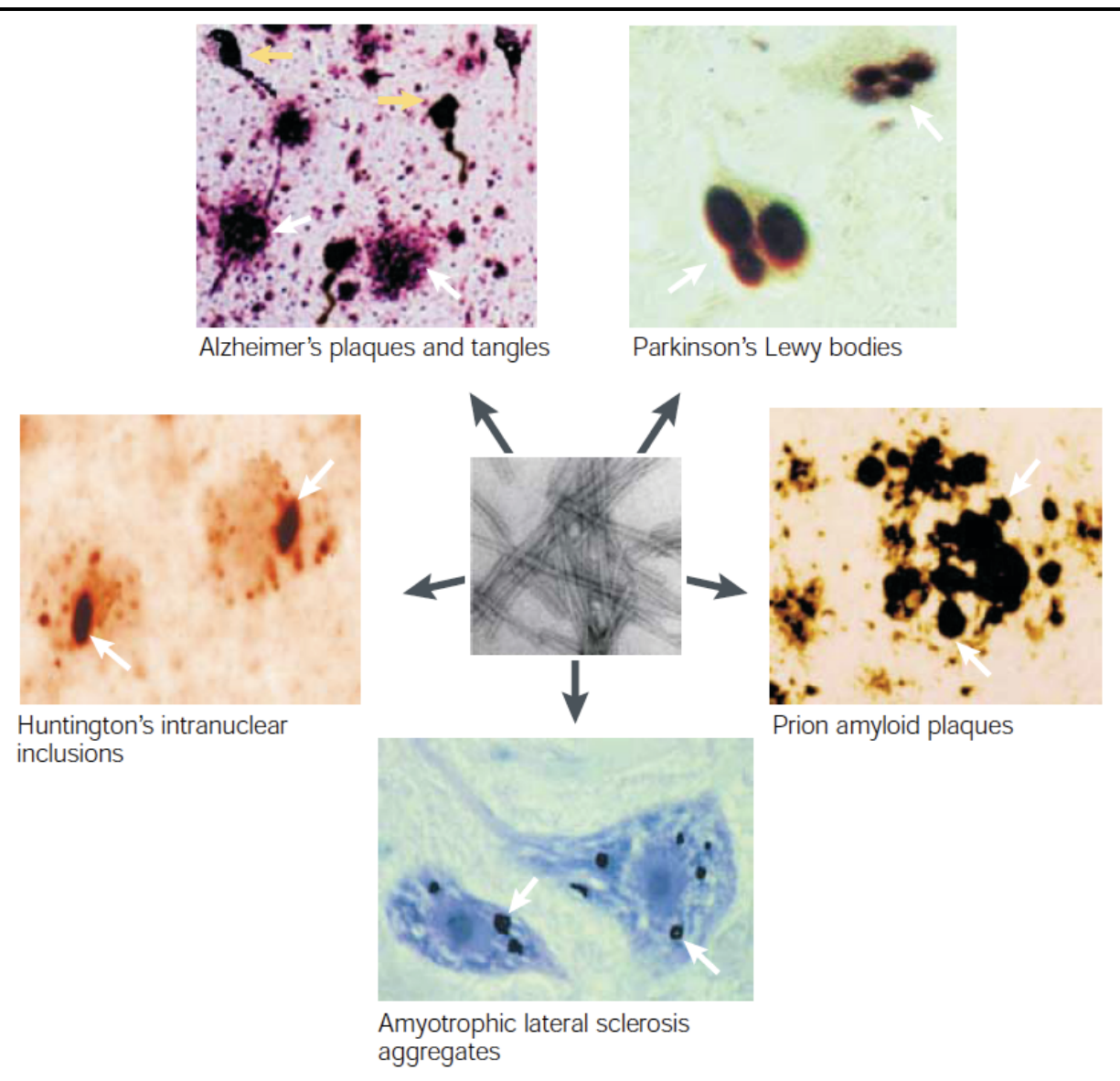

Figure 4: Amyloid aggregates of misfolded proteins in the neurodegenerative diseases

Amyloid plaques (white arrows) in different neurodegenerative disorders have fibrillary deposits of misfolded proteins. In spite of different protein compositions, the ultrastructure of these deposits is similar and composed mainly of a network of protein fibrils (electron micrograph of fibrils in the centre). Cellular localization of these aggregates varies in different neurodegenerative diseases: extracellular amyloid plaques and intracellular neurofibrillar tangles (yellow arrows) in Alzheimer's disease, intracytoplasmic aggregates in Parkinson's disease and Amyotrophic lateral sclerosis, 
intranuclear inclusions in Huntington's disease, and extracellular prion amyloid plaques in Spongiform encephalopathy and Creuzfeldt Jacob disease. Adapted figure from Soto, 2003.

$\alpha \mathrm{S}$ has little or no detectable secondary structure in its native form in solution and hence, it is referred to as natively unstructured (Bernado et al., 2005; Cookson, 2005; Ulmer et al., 2005). $\alpha S$ with its natively unfolded structure and hydrophobic NAC region has a high propensity to misfold into the conformations that have a higher tendency to aggregate. The process of misfolding and aggregation is aggravated in PD and other synucleinopathies either by the missense mutations in $\alpha \mathrm{S}$ gene or by sporadic factors (reviewed in Lansbury and Lashuel, 2006; Moore et al., 2005). Generally, the genetic perturbations in $\alpha \mathrm{S}$ gene or familial forms of PD have early-onset and greater severity than the sporadic cases and they are also associated with a greater load of protein aggregates (Hardy and Selkoe, 2002). Recently, point mutations discovered in the $\alpha \mathrm{S}$ gene as shown in Figure 3 (A53T- $\alpha \mathrm{S}$ in Greek kindred; A30P- $\alpha \mathrm{S}$ in German kindred; and E46K- $\alpha \mathrm{S}$ in Spanish kindred) and triplication of the $\alpha \mathrm{S}$ locus (in Iowa kindred) (Kruger et al., 1998; Polymeropoulos et al., 1997; Singleton et al., 2003) alter the fibrillation propensity of $\alpha S$ that subsequently results in the autosomal dominant PD. In addition, recent genome-wide association studies have unequivocally revealed that SNP (single nucleotide polymorphism) variations within the $\alpha \mathrm{S}$ and LRRK2 (leucine-rich repeat kinase 2) gene loci contribute to the etiology of sporadic PD (Simon-Sanchez et al., 2009).

High-resolution three-dimensional structures of the fibrils of $\alpha \mathrm{S}$ and other proteins involved in neurodegeneration commonly have a canonical 'cross- $\beta$ ', structure, the structural fingerprint of amyloid fibrils. The $\beta$ strands from individual misfolded protein monomers are arranged perpendicular to the axis of cross- $\beta$ structure and stabilize it by the inter- and intra-molecular interactions (Antzutkin et al., 2000; Balbach et al., 2002; Heise et al., 2005; Petkova et al., 2002). Fibrils formed by same protein or peptide can exhibit conformational polymorphism (Chiti and Dobson, 2006; Heise et al., 2005; Vilar et al., 2008). 
Nevertheless, it is worth noticing here that the proteins with completely different primary sequence can fold into the same three-dimensional structure in their aggregated form (Figure 5).

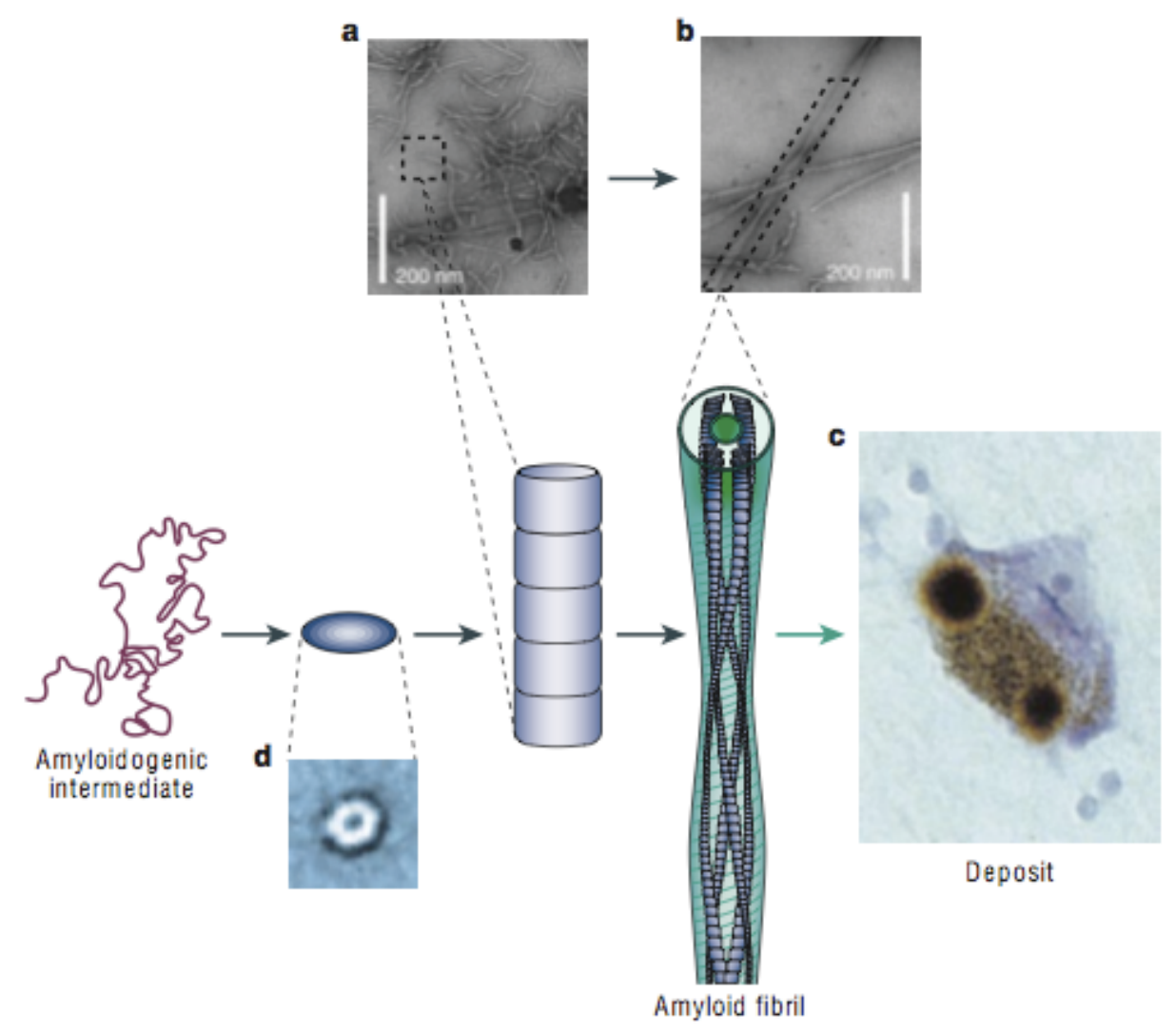

Figure 5: Schematic representation of the general mechanism of aggregate formation

Unfolded or partially unfolded proteins associate with each other to form small, soluble pre-fibrillar oligomers that undergo further assembly into protofibrils or protofilaments (a) and then mature fibrils (b, top electron microscope images). The fibrils often accumulate in plaques or other structures such as the Lewy bodies associated with Parkinson's disease (c, electron microscope image on right). Some of the early aggregates seem to be amorphous or micellar in nature, although other soluble pre-fibrillar oligomers form ring-shaped species with diameters of approximately $10 \mathrm{~nm}$ (d, electron microscope image). Adapted figure from Dobson, 2003. 


\subsection{Soluble pre-fibrillar oligomers in neurodegeneration - revisiting the amyloid hypothesis}

The recent decade of research on neurodegenerative diseases has resulted in shaping the "amyloid hypothesis" to define a precise relationship between protein aggregation and neurodegeneration. The amyloid hypothesis postulates that aggregation of proteins (for example, $\alpha \mathrm{S}$ ) triggers a cascade of events that would finally result in the neurodegeneration and disease (Hardy and Selkoe, 2002). In addition, factors that promote increased expression and decreased degradation of aggregate-prone proteins (like $\alpha \mathrm{S}$ ) are sufficient to cause neurodegeneration (Barrachina et al., 2006; Lansbury and Brice, 2002; Leroy et al., 1998). This hypothesis has drawn evidence and support mainly from three disciplines: neuropathology, genetics, and biophysics. It has withstood concerted efforts to disprove it and evolved along with the new scientific developments in understanding of the in vitro aggregation and in vivo neuropathological studies.

There is indeed an existing correlation between the presence of LBs containing aggregated proteins in the brain and the neurodegenerative disorders. But the finding that amyloid plaques are also found throughout the cortex of many cognitively normal 70-year-old people has confounded the interpretation of relationship between the protein aggregation and neurological disease (Dickson et al., 1992). Though available evidence strongly suggests formation of fibrillar aggregates as an epiphenomenon of the disease, logical interpretation based on recent in vitro and in vivo studies predicts that pre-fibrillar intermediates in the aggregation pathway could be the apparent toxic species involved in neurodegeneration (Conway et al., 2000; Hartley et al., 1999; Lansbury and Lashuel, 2006; Rochet et al., 2000; Volles and Lansbury, 2002; Walsh et al., 2002). Based on this interpretation, it is conceivable that genetic and sporadic factors that promote/accelerate formation of pre-fibrillar oligomers or aggregation intermediates may not only result in early-onset of the disease but also increase the formation of fibrillar aggregates (Figure 6). 


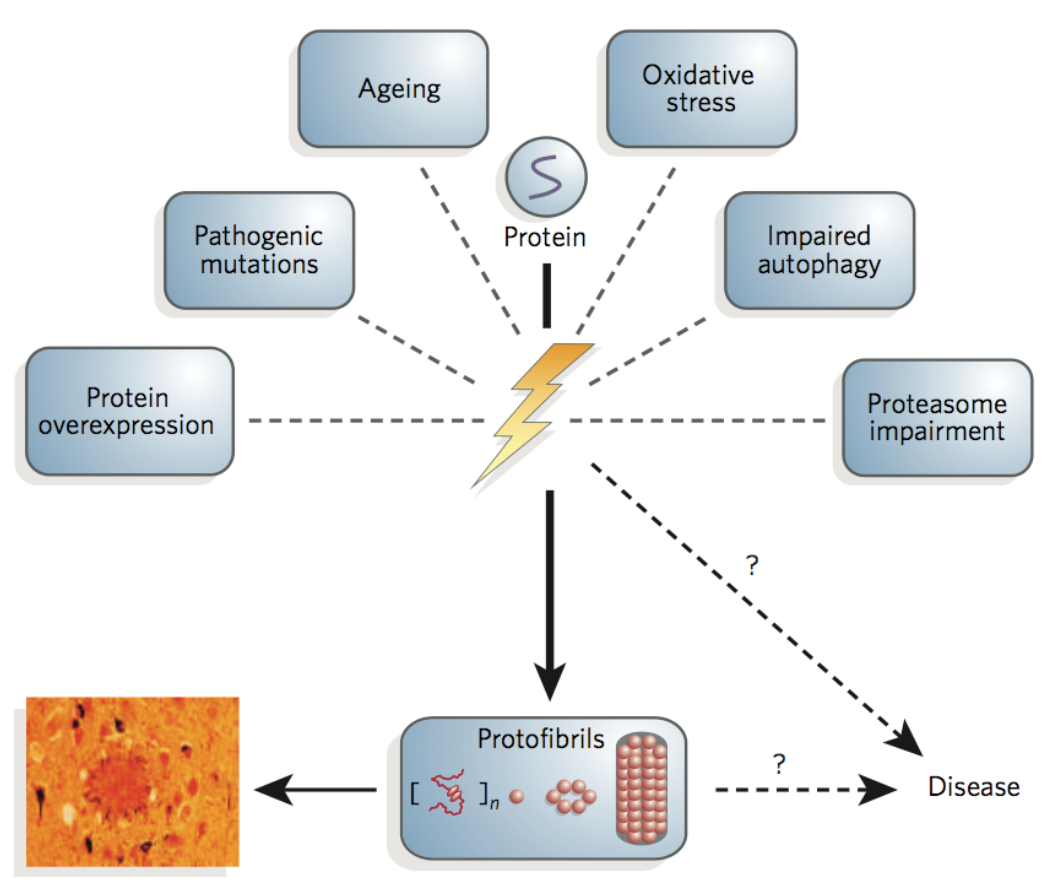

Figure 6: Factors promoting the formation of pre-fibrillar oligomers may cause early-onset of the disease

A number of factors have been proposed to trigger protein oligomerization and disease. Whether pre-fibrillar aggregates are the cause of disease is still uncertain, but circumstantial evidence supports a pathogenic role for these structures. Adapted figure from Lansbury and Lashuel, 2006.

In contrast to the unusual stability of fibrillar structures, diffusible prefibrillar oligomers are not stable and are proven to be very difficult to extract and characterize from the brains (reviewed in Lansbury and Lashuel, 2006). Besides this, the path towards ascertaining the role of soluble pre-fibrillar oligomers in neurodegeneration is compounded by the dynamic nature of protein aggregation and the technical challenges of advanced imaging methods for correlating disease progression in vivo to the protein aggregation in vitro. 


\subsection{Pathogenicity of pre-fibrillar soluble oligomers}

The above-mentioned paradigm shift of focus towards pre-fibrillar oligomers in understanding the nature and mechanisms of pathogenesis involved in neurodegenerative disorders has spurred numerous new scientific studies. Despite tremendous progress, there is by now still no conclusive evidence that pinpoints the nature of toxic species in the aggregation pathway. However, there are some common structural and functional features shared by pre-fibrillar oligomers of different amyloid proteins that could be useful to decipher the possible mechanisms involved in toxicity (reviewed in Lashuel and Lansbury, 2006).

Soluble pre-fibrillar oligomers of misfolded proteins can diffuse and interfere with many neuronal functions. They can adversely affect synaptic physiology, structure and function, thereby changing the overall plasticity and integrity of individual neurons and neuronal networks. Studies done on the soluble A $\beta-42$ oligomers formed by the Amyloid- $\beta$ (A $\beta)$ and polyglutamine-rich Huntingtin oligomers have demonstrated that oligomerization of misfolded protein is harbinger of the cascade of events that finally result in dementia and cognitive dysfunction (reviewed in Haass and Selkoe, 2007). In addition, the prefibrillar oligomers formed by amyloid proteins like $\alpha \mathrm{S}$ (PD and diffuse Lewy body disease), A $\beta$ (Alzheimer's disease), Superoxide dismutase-I (Amyotrophic lateral sclerosis), Prion protein (Creutzfeldt-Jakob disease, bovine spongiform encephalopathy), and Huntingtin (Huntington's disease) are shown to form porelike structures (Lashuel and Lansbury, 2006). The pore-like structures formed by amyloid oligomers of pathogenic proteins are shown to affect the integrity of lipid membrane. Thus it is hypothesized that they may impair the functions of neuronal, mitochondrial membranes and dopamine vesicles (see Figure 7).

In addition, pre-fibrillar oligomers can also promote their own accumulation by inhibiting proteasomal activity (Figure 7; Lashuel and Lansbury, 2006). Experimental support for the hypothetic scheme outlined in the Figure 7 comes mainly from in vitro studies and the studies done in cell culture models (Ding et al., 2002; Gosavi et al., 2002; Leroy et al., 1998; Rubinsztein, 2006; Sharon et al., 
2003a; Sharon et al., 2003b). Pre-fibrillar oligomers of wild type/WT- $\alpha \mathrm{S}$ and three familial PD $\alpha$ S mutants, A30P- $\alpha \mathrm{S}, \mathrm{A} 53 \mathrm{~T}-\alpha \mathrm{S}$, and E46K- $\alpha \mathrm{S}$, form annular and tubular structures akin to protein channels or pores (Kim et al., 2009; Lashuel and Lansbury, 2006). However, in vivo pathogenicity of the soluble pre-fibrillar oligomers of $\alpha \mathrm{S}$ has not been tested in any animal model system.

Considering these important developments, it is very challenging to correlate the in vitro pre-fibrillar oligomer formation and their in vivo biological significance in PD pathogenesis. Major impediments to address this problem are the dynamic nature of protein aggregation process and the extremely transient and labile properties of the pre-fibrillar oligomeric intermediates. As soon as the concentration of so-called 'on-pathway' oligomeric intermediates reaches certain threshold, they are rapidly converted into fibrils (Kim et al., 2009). Contrary to the on-pathway amyloid $\alpha S$ oligomers, off-pathway oligomers don't seed the fibrillation of WT- $\alpha \mathrm{S}$ and they are found to be innocuous. This additional on/offpathway feature of aggregation intermediates further complicates the design of experiments to resolve the identity of pathogenic species in the PD pathogenesis.

(on the next page)

\section{Figure 7: Schematic depiction of the potential pathogenic mechanisms in PD}

Several factors have been shown to play a role in initiating and/or accelerating $\alpha S$ aggregation, consequently promoting the pre-fibrillar oligomer formation. Factors such as increased expression, missense mutations, oxidative stress induced modifications such as cross-linking, and phosphorylation at S129 influence the aggregation of $\alpha \mathrm{S}$ to promote formation of pre-fibrillary oligomers (Chen and Feany, 2005; Chen et al., 2009; Fujiwara et al., 2002; Smith et al., 2005). In addition, pre-fibrillar oligomers can promote their accumulation by inhibiting the proteasome. Pore-like structures formed by amyloid oligomers can affect the membranes of mitochondria and DA vesicles leading to release of reactive oxygen species (ROS) and other pro-apoptotic factors (Abou-Sleiman et al., 2006). In addition, proteins that have a role in regulating the $\alpha \mathrm{S}$ levels are known to be the risk factors for PD (for example, neuronal UCH-L1 (Ubiquitin C-terminal hydrolase1), and Parkin (E3-ubiquitin ligase)) (Barrachina et al., 2006; Leroy et al., 1998). Taken 
together, experimental evidence generated from the above studies suggests possible role of soluble pre-fibrillar oligomers of $\alpha \mathrm{S}$ in PD pathogenesis.

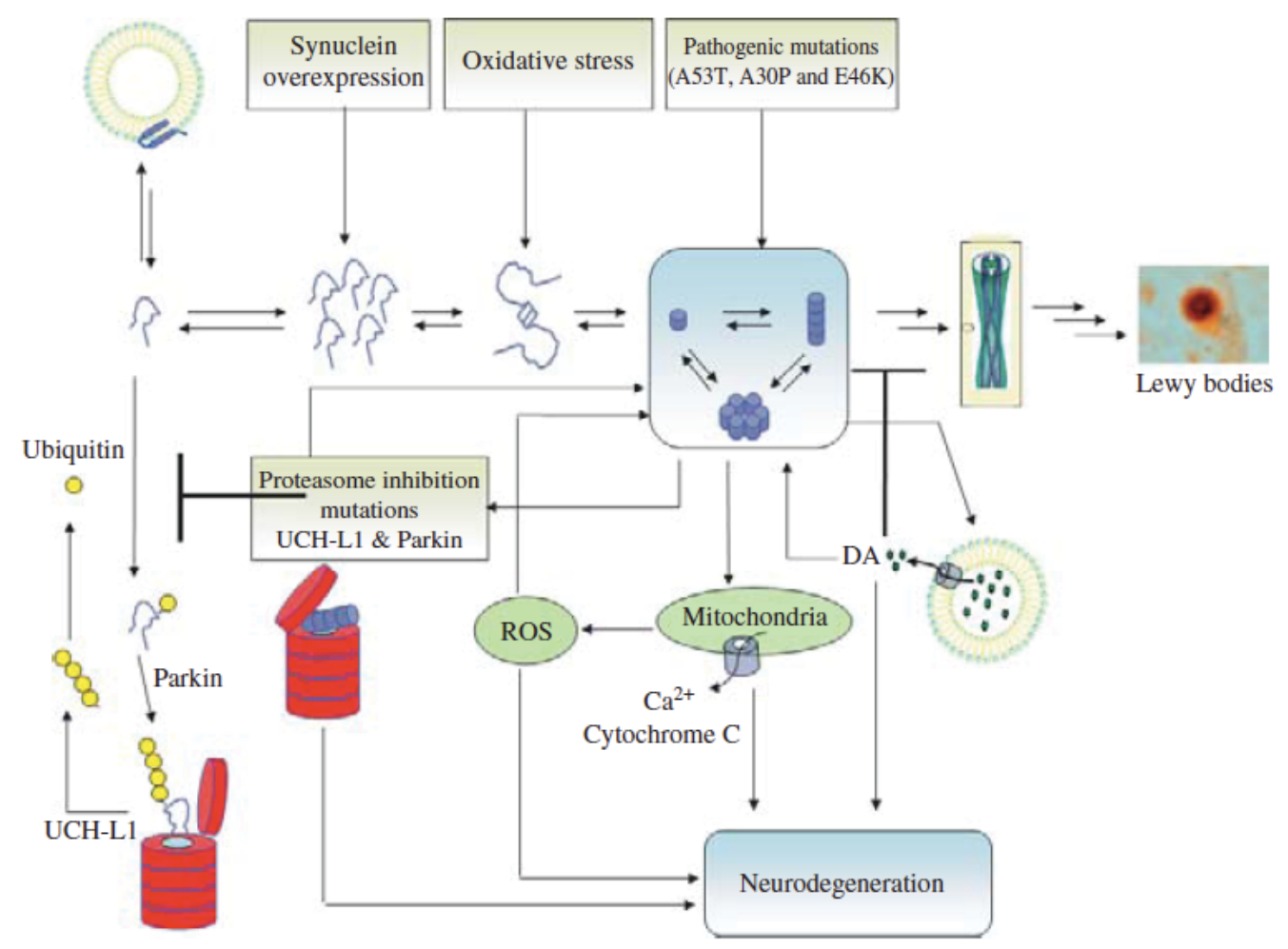

\subsection{Drosophila model for Parkinson's disease}

The fly Drosophila melanogaster, below referred to simply as Drosophila or "fly", has enabled significant advances in neurodegenerative research, notably in the identification of genes that are required to maintain structural integrity of the brain. Gene discovery is quick and straightforward in flies. Furthermore, the central nervous system of invertebrates and vertebrates share a common evolutionary origin, and the fly has been used successfully for the genetic analysis of complex behaviors ranging from sleep to learning and memory to aggression. Of the human protein sequences associated with disease in the Online Mendelian Inheritance (OMIM) database, 74\% have highly related sequences in the fly 
genome (http://superfly.ucsd.edu/homophila/; Chien et al., 2002). Moreover, a number of dominantly inherited human neurodegenerative diseases, such as those caused by polyglutamine repeat expansions, have been successfully modeled in Drosophila by transgenic expression of human disease genes (reviewed in Lessing and Bonini, 2009).

Drosophila contains no endogenous $\alpha \mathrm{S}$ or $\alpha \mathrm{S}$-like genes in its genome (Auluck and Bonini, 2002; Feany and Bender, 2000). Feany and Bender developed the first Dorosophila PD model by introducing human $\alpha \mathrm{S}$ gene into the wildtype fly genome ("humanized flies"). In their study, pan-neuronal overexpression of human wildtype (WT- $\alpha \mathrm{S}$ ) and mutant $\alpha \mathrm{S}$ (A53T- $\alpha \mathrm{S}, \mathrm{A} 30 \mathrm{P}-\alpha \mathrm{S}$ ) using the UAS/Gal4 bipartite system (Section 2.2.3) has resulted in the human PD-like features in flies (Feany and Bender, 2000). This initial study spearheaded a flurry of investigations further on addressing various aspects of the neurodegeneration in PD. $\alpha \mathrm{S}$ expressed in Drosophila has been shown to undergo the similar posttranslational modifications (for example, S129 and Y125 phosphorylation) and processing as observed in human (Chen and Feany, 2005; Chen et al., 2009). Humanized Drosophila expressing $\alpha \mathrm{S}$ mutants also show motor deficits in the climbing assay, reduced lifespan in survival assay, and degeneration of dopaminergic neurons in an age dependent manner (Feany and Bender, 2000).

Climbing (= negative geotaxis) is the most commonly assayed behavior with respect to fly neurodegeneration. It is used for three reasons: (i) as a test of mobility, (ii) it can reflect ataxia that is common in human degenerative diseases, and (iii) a large number of flies can be tested at once. Generally, all neurodegenerative mutants have a shortened lifespan; therefore a straightforward first look at a mutant can be obtained with a survival curve. Also, the assay is also easy to perform and the observed behavioral deficit correlates well with the human disease. Drosophila also has easily identifiable and countable dorsomedial (DM) and dorsolateral (DL) clusters of the dopaminergic (DA) neurons in the adult brain, which can be immunostained with tyrosine hydroxylase antibody 
(Periquet et al., 2007). The DM and DL clusters are also known to be sensitive to the $\alpha$ S-mediated toxicity (Periquet et al., 2007; Sang et al., 2007). Though significant loss of the DA neurons in the Substantia Nigra is a common clinical feature in human PD, neurodegeneration is not restricted to the DA neurons but extends to other regions of brain as the disease progresses (Farrer, 2006).

The Drosophila model also provides a clean genetic background, as it doesn't have endogenous $\alpha \mathrm{S}$ in its wildtype genome. This experimental situation allows then investigating the dominant-gain of toxic functions of the human $\alpha \mathrm{S}$ mutations in comparison with the effect of wildtype- $\alpha \mathrm{S}$ expression in the fly. Fundamental biological pathways in flies, such as signaling, metabolism, innate immunity, learning and memory, sleep as well as circadian rhythms, share significant similarity to the mammalian pathways (Hendricks and Sehgal, 2004). As a result, the use of Drosophila as a model system also helps to understand the mechanisms responsible for PD pathogenesis. And of course, amenability and ease of Drosophila genetics further makes it a best system to screen for the candidate genes modifying $\alpha \mathrm{S}$ toxicity. In fact, Drosophila is so far is one of the PD model organism that has shown significant and consistent similarity to the human PD phenotype (reviewed in Farrer, 2006; Maries et al., 2003).

As mentioned in the previous sections, a million dollar question that still remains to be answered in the emerging field of PD pathogenesis is the precise identity and nature of pathogenic species in the aggregation pathway of $\alpha \mathrm{S}$. First step towards answering this question involves finding an animal model system that can reliably translate the pattern of observations made from in vitro aggregation experiments into the in vivo toxicity readout. We can then use that model system to correlate the in vitro aggregation properties of $\alpha \mathrm{S}$ variants (for e.g. $\alpha \mathrm{S}$ variants that form more pre-fibrillar oligomers and no fibrils) to the in vivo neurotoxicity. In the current study, I have generated an improved Drosophila PD model using the Phi-C31 site-specific integration system (Sections 2.2.2; 3.3) to correlate the in vitro pre-fibrillar oligomer formation of $\alpha \mathrm{S}$ to the in vivo neurotoxicity. The advantage of this system is that transgenes can be inserted into 
the same chromosomal location of the flies. This way, possible 'position effects' resulting from the $\alpha \mathrm{S}$ gene insertion into different locations of the fly genome is circumvented. The site-specific integration of the $\alpha \mathrm{S}$ gene consequently allows us to directly compare the toxicity of different $\alpha \mathrm{S}$ variants in vivo because of the similar expression levels of the inserted transgenes.

\subsection{Understanding motor and non-motor symptoms of PD in the Drosophila model}

In most PD animal models including Drosophila, studying motor dysfunction is the main focus to understand PD pathogenesis. Since motor symptoms are considered to arise as a direct result of the loss of DA neurons in brain which is easy to observe and score. Partly it is also because of the popularity of Parkinson's disease as a movement disorder. As already outlined above, the climbing assay is the most commonly used tool to check the mobility and motor integrity of PD (Lessing and Bonini, 2009). Here, I present a new PD Drosophila model with which I have tested both the negative geotaxis and positive phototaxis in a specially devised climbing assay apparatus (Sections $2.3 .1 ; 3.4$ ) and I have also monitored the fly locomotor activity using the Drosophila Activity Monitors (Sections 2.3.3; 3.9).

Strikingly, understanding non-motor symptoms like sleep disorders, circadian changes, and cognitive dysfunction are not given much emphasis in the animal models generally used, as they are relatively tough and intricate to study. But the quality of life in human PD patients is severely affected by these nonmotor symptoms. Improving non-motor symptoms like sleep will not only increase the quality of life of patients but also helps them to recover from circadian and cognitive dysfunction (Pallier et al., 2007; Reddy and O'Neill, 2010). Conserved cardinal features of sleep and circadian pathways in Drosophila (reviewed in Colwell, 2007; Hendricks and Sehgal, 2004) make it an indispensable and invaluable system to both understand and improve non-motor symptoms in PD pathogenesis. With the help of the newly generated Drosophila PD model system presented here, which uses site-specific recombination events to 
insert mutant $\alpha \mathrm{S}$ variants into the same location of the fly genome (Sections 2.2.2; 3.3), I have pioneered to study both the motor and non-motor symptoms such as sleep and circadian rhythms in order to correlate in vitro pre-fibrillar oligomer formation with the in vivo neurotoxicity of distinct $\alpha \mathrm{S}$ variants in PD pathogenesis.

\subsection{Aims of this study}

Considering recent developments in the field of PD pathogenesis when I have started this work, my aim was to investigate and ascertain the nature and identity of pathogenic species in the aggregation pathway of $\alpha \mathrm{S}$. In my notion, results from this huge scientific stride would help to resolve a longstanding debate in the field of PD pathogenesis, and funnel the focus of concerted efforts in a specific direction to understand the mechanisms of PD pathogenesis. I have planned to undertake a reverse genetics approach to investigate this problem in a systematic fashion. Initially, we need to resolve the question of whether the prefibrillar oligomers or the fibrils of $\alpha \mathrm{S}$ are potent pathogenic species in aggregation pathway of $\alpha \mathrm{S}$. Since there has been accumulating evidence that pre-fibrillar oligomers are the potential pathogenic species in the neurodegenerative diseases, like in Alzheimer's and Huntington's disease, my primary goal was to test whether this paradigm also holds true for the Parkinson's disease.

My strategy to achieve this goal involved the rational design of different $\alpha \mathrm{S}$ variants based on structural information and testing their aggregation properties in vitro. This part of the work was performed in collaboration with Christian Griesinger's and Markus Zweckstetter's lab. After generating and characterizing different $\alpha \mathrm{S}$ variants that can form more pre-fibrillar oligomers and less or even no fibrils, our idea was to correlate the in vitro soluble pre-fibrillar oligomer formation to the in vivo neurotoxicity in different model systems including $C$. elegans, Drosophila and mammalian neurons. My specific aim was to generate a Drosophila model for PD in order to critically test whether the in vitro prefibrillar oligomer formation can be correlated with in vivo toxicity of different $\alpha \mathrm{S}$ 
variants that were generated by the rational design. By using Drosophila as a model for PD, I also performed studies concerned with both non-motor and motor symptoms of human PD (Section 1.8). The study was designed to better understand PD pathogenesis as a starting point for devising novel therapeutic interventions. 


\section{MATERIALS AND METHODS}

\subsection{Molecular Biology}

\subsubsection{Polymerase Chain Reaction (PCR)}

PCR was used to amplify or mutate DNA fragments. It was carried out according to a standard protocol (Ausubel, 1999). Annealing temperatures and extension times were adjusted to fit the respective primer melting temperature and the length of the expected PCR product. The typical protocol involved 30-35 cycles (at annealing temperature $55^{\circ} \mathrm{C}$ and extension time $1 \mathrm{~min} 30 \mathrm{sec}$ ) in a PTC-200 DNA Engine Cycler (Biorad, Hercules, USA).

\subsubsection{DNA Sequencing}

DNA sequencing was performed by the MWG Company (Ebersberg, Germany). DNA and primers were added according to the instructions given by the company on how to prepare a sample for the sequencing reaction.

\subsubsection{Restriction digest of DNA}

Restriction endonucleases (New England Biolabs, Ipswich, USA or Fermentas Burlington, Canada) were used according to the manufacturer's instructions. For cloning, PCR-products or preparative amounts of plasmid DNA (10-20 $\mu \mathrm{g})$ were incubated overnight with 10-folds excess of enzyme. 


\subsubsection{Sample preparation of single fly PCR}

Single adult flies were homogenized on ice in $50 \mu \mathrm{l}$ squashing buffer $(10 \mathrm{mM}$ Tris- $\mathrm{HCl} \mathrm{pH}$ 8.2, $1 \mathrm{mM}$ EDTA, $25 \mathrm{mM} \mathrm{NaCl}, 200 \mu \mathrm{g} / \mathrm{ml}$ proteinase $\mathrm{K}$ ). The homogenate was incubated at $37^{\circ} \mathrm{C}$ for $30 \mathrm{~min}$ and proteinase $\mathrm{K}$ activity quenched by subsequent heat treatment $\left(95^{\circ} \mathrm{C}\right.$ for $\left.2 \mathrm{~min}\right)$. After sedimentation by a short centrifugation step, the supernatant was then directly used for PCR.

\subsubsection{DNA extraction from agarose gels}

Ethidium Bromide stained DNA was excised from the agarose gel using a clean scalpel and extracted using the QIAquick Gel Extraction Kit (QIAGEN, Hilden, Germany) as described by the manufacturer.

\subsubsection{Dephosphorylation of vector DNA}

In order to minimize self-ligation of vector DNA, the $5^{\prime}$ phosphate group was removed by Alkaline Phosphatase treatment. Restricted vector DNA was incubated for $30 \mathrm{~min}$ with $1 \mu \mathrm{l}$ of Shrimp Alkaline Phosphatase (SAP, USB, Staufen, Germany) and the appropriate volume of dephosphorylation buffer (USB) as outlined by the manufacturer. Dephosphorylated DNA was subsequently purified using the QIAspin Nucleotide Removal Kit (Qiagen, Hilden, Germany) according to the provided manual.

\subsubsection{DNA ligation}

Ligation was performed with T4 DNA ligase (Fermentas, Burlington, Canada). A total of 100ng of DNA (molar ratio of insert to host vector DNA of 3:1) was incubated with $1 \mu \mathrm{T} 4$ ligase in $10 \mu \mathrm{l}$ reaction volume (overnight, $18^{\circ} \mathrm{C}$ ) as recommended by the manufacturer.

\subsubsection{Transformation of bacterial cells}

Escherichia coli DH5 $\alpha$ or Top10 cells were obtained from Invitrogen (Karlsruhe, Germany). Chemically competent cells were prepared according to a standard protocol (Inoue et al., 1990). For transformation of cells by DNA present in the ligation reactions, an aliquot of chemically competent cells was added to 
approximately $100 \mathrm{ng}$ of plasmid DNA followed by $30 \mathrm{~min}$ incubation on ice. Cells were then heat shocked in a water bath at $\left(42^{\circ} \mathrm{C}, 45 \mathrm{~s}\right)$ and immediately transferred back on to ice. After 2 min on ice, Luria-Bertani (LB) medium (Invitrogen, Karlsruhe, Germany) was added, and the cells were allowed to recover for $30 \mathrm{~min}$ at $37^{\circ} \mathrm{C}$. The cells were distributed on LB agar plates containing antibiotics and incubated overnight at $37^{\circ} \mathrm{C}$.

\subsubsection{Preparation of plasmid DNA}

Plasmid DNA was purified using the QIAGEN Mini or Maxi Kits (QIAGEN, Hilden, Germany) following the manufacturer's protocol

\subsubsection{DNA preparation for embryo injections}

Targeting plasmid construct containing attB site and the vector DNA elements were cloned and purified using the Quiagen Midi-prep DNA kit (Qiagen, Hilden, Germany) and the protocol provided by the manufacturer. Purified DNA was diluted with water to a final concentration of $200 \mathrm{ng} / \mu \mathrm{l}$. DNA of the targeting construct (i.e. the "transgene") was injected into the embryos which were homozygous for both a attP landing site in a defined position of the genome and the germline specific Phi-C31 integrase provided by an integrated transgene (Bischof et al., 2007).

For details see http://flyc31.frontiers-in-genetics.org/sequences_and_vectors.php

\subsubsection{Preparation of fly head extracts}

Flies (wildtype or mutants) were frozen and approximately $50 \mu 1$ of fly heads of the different genotypes were separated and collected. Heads were homogenized in three volumes of the ice-cold Head Extraction Buffer (containing $100 \mathrm{mM} \mathrm{KCl}$, 20mM Hepes, 5\% Glycerol, 10mM EDTA, 0.1\% Trition X-100, 1mM DTT, Sigma cocktail protease inhibitor, $\mathrm{PH}$ - 7.5) as described (Nawathean and Rosbash, 2004). The homogenate was subsequently centrifuged for $5 \mathrm{~min}$ to obtain the supernatant. After removal of the supernatant, protein concentration was determined using BCA (Bicinchoninic Acid) method using BCA Protein Assay Kit (Pierce Biotechnology, Rockford, USA) according the manufacturer's 
manual. For comparison, $\sim 50 \mu \mathrm{g}$ of total protein extracts was mixed with the $2 \mathrm{X}$ SDS sample buffer and proteins were subsequently separated by SDS PAGE (see Section 2.1.12).

\subsubsection{Polyacrylamide Gel Electrophoresis (PAGE)}

Samples were separated in a 15\% denaturing Tris/Tricine SDS polyacrylamide gel electrophoresis system, as described (Schagger and von Jagow, 1987). The separation gel contained 15\% bis-acrylamide (Rotiphorese Gel 30, Roth GmbH, Karlsruhe, Germany), $1 \mathrm{M}$ Tris (pH 8.45), 0.1\% SDS, 10\% glycerol; the stacking gel contained 4\% bis-acrylamide $1 \mathrm{M}$ Tris (pH 8.45), 0.1\% SDS. Ammonium persulfate and TEMED (N, N, N', N'-Tetramethylethylene-diamine) were added for polymerization and cross-linking of the bis-acrylamide. Before loading the protein samples onto the gel, they were boiled for $5 \mathrm{~min}$. $5 \mu$ PageRuler prestained protein ladder solution (Fermentas, Burlington, Canada) was used for each gel as marker for an approximate sizing of the proteins. Separation was performed in a discontinuous buffer system with a $0.2 \mathrm{M}$ Tris $(\mathrm{pH} 8.9)$ solution in the tank and a $0.3 \mathrm{M}$ Tris ( $\mathrm{pH} 8.45$ ), $0.03 \%$ SDS solution as running buffer.

\subsubsection{Immunoblotting}

After separation, proteins were transferred from the gel to nitrocellulose membranes (Amersham Biosciences, Freiberg, Germany) in a sandwich setting (BioRad, Muenchen, Germany) for $1 \mathrm{~h}$ at $350 \mathrm{~mA}$ following otherwise a standard protocol (Ausubel, 1999). After transfer, membranes were blocked with 5\% BSA in TBST (50 mM Tris pH 7.4, $150 \mathrm{mM} \mathrm{NaCl}, 0.1 \%$ Tween-20), incubated with primary antibodies $\left(4^{\circ} \mathrm{C}\right.$, overnight), washed with TBST, incubated with HRP (Horse Radish Peroxidase)-conjugated secondary antibodies (room temperature; 2 h), washed and then subjected to ECL (a highly sensitive enhanced chemiluminiscent substrate for detecting HRP on immunoblots) detection. The activity of HRP-conjugated secondary antibodies (Jackson labs, West Grove, USA) was determined using ECL Western Blotting Substrate (Pierce Biotechnology, Rockford, USA). Primary antibodies, mouse monoclonal anti- $\alpha$ Tubulin antibodies (clone DM1A from Sigma-Aldrich, Munich, Germany) and 
rabbit polyclonal $\alpha$-Synuclein antibodies (Anaspec, Fremont, USA) were diluted 1:2,000- and 1:300-fold, respectively. HRP conjugated secondary antibodies were used at the dilution of 1: 3,000.

\subsubsection{Dot blotting}

Purified protein samples of $\alpha \mathrm{S}$ wild type and $\alpha \mathrm{S}$ variants were spotted on to the nitrocellulose membranes for dot blotting as described (Jahn et al., 1984). Dot blotting was done using the anti-oligomer antibody/A11 (Invitrogen Corporation, Camarillo, USA) antibody as described (Kayed et al., 2003).

\subsection{Fly Techniques}

\subsubsection{Maintenance of flies}

Flies were kept and propagated on standard fly food used in the Lab, a corn floursoya flour-molasses medium that is supplemented with dry yeast (Casteggio Lieviti srl, Casteggio, Italy) at $25^{\circ} \mathrm{C}$ and $20-30 \%$ humidity. For measuring circadian locomotor activity rhythms and sleep assays, flies were kept in a $12 \mathrm{~h}$ light/12h dark (LD) cycle unless mentioned differently in the 'results' section.

\subsubsection{Generation of stable transgenic fly lines}

The site-specific PhiC31integrase/ $\phi C 31$ integrase recombination system (Bischof et al., 2007) was used to generate transgenic flies which contain the transgene in the same chromosomal location. PhiC31 site-specific integration system depends on the presence of bipartite sequences, the so-called attachment site $\mathrm{P}$ (attP) and the attachment site B (attB) integrated into the fly genome that contain also a PhiC31integrase gene to carry out irreversible integration between attB and attP sites $($ attB + attP $+\phi C 31$ integrase $=>$ attR + attL $+\phi C 31$ integrase $)$. For details see Bischof et al., 2007.

The DNA constructs used as transgenes were prepared by cloning the cDNAs of $\alpha S$ variants into the GAL4-responsive pUAST expression vector (Bischof et al., 
2007) containing the attachment site B (attB). The resulting plasmids were injected into the fly embryos, which were double homozygous for both attP (attachment site P) site and germ-line-specific $\phi C 31$ integrase. The genomic location of the attP landing site used for integration was mapped to the $3 \mathrm{R}-86 \mathrm{Fb}$ position in the genome ( $\mathrm{ZH}-86 \mathrm{Fb}$ line) (Bischof et al., 2007). For details see http://flyc31.frontiers-in-genetics.org/landing_platforms.php. $\quad$ Site-specific insertions of the transgenes were verified by single fly PCR using primer pairs of the sequence 5'ACT GAA ATC TGC CAA GAA GTA 3' and 5 GCA AGA AAG TAT ATC TCT ATG ACC 3', respectively.

\subsubsection{Ectopic gene expression via the UAS/GAL4 system}

The UAS/GAL4 system allows for ectopic induction of genes in a spatially and temporally controlled fashion (Brand and Perrimon, 1993). This two-component system is borrowed from yeast where the transcription factor GAL4 binds to its target Upstream Activating Sequence (UAS) to activate transcription (Brand and Perrimon, 1993). A transgenic fly carrying a specific enhancer element upstream of the gal4 gene, when mated with a transgenic fly carrying a coding sequence fused downstream of the UAS, results in progeny, which expresses the UAS-fused coding region in response to GAL4 activity driven by an enhancer element that directs the spatio-temporal pattern of gal4 expression.

\subsubsection{Antibody (Ab) staining of adult fly brains}

Whole-mount adult fly brains from the 2day and 30day old animals were prepared and immunostained according to the previously published protocol ( $\mathrm{Wu}$ and Luo, 2006). Rabbit anti-tyrosine hydroxylase (TH) (dilution 1:150; Chemicon International, Temecula, USA) was used to positively stain the Dopaminergic (DA) neurons, and Mouse anti-nc82 (dilution 1: 200; Developmental Studies Hybridoma Bank, University of Iowa, Iowa City, IA) was used as a counter stain. From the confocal sections of fly brains of different genotypes, dorsomedial (DM) and dorsolateral (DL) clusters of DA neurons were defined and counted as described (Cooper et al., 2006; Sang et al., 2007). 'Cell counter' plug-in as 
provided by the ImageJ64 software was used for counting the DA neurons in the stacks of confocal images (http://rsbweb.nih.gov/ij/index.html).

\subsubsection{Dissection of larval NMJs (Neuromuscular Junctions)}

Midstage third-instar larvae were put on a dissection plate with both ends fixed by fine pins and then covered by a drop of ice-cold hemolymph-like saline (HL-3) (low calcium saline solution containing 0-0.3mM calcium (Broadie, 2000; Stewart et al., 1994). Dissection scissors were used to first make a small hole at the dorsal midline of the larva near the posterior end, which was then extended along the dorsal midline to the anterior end. The epidermis was stretched flat and pinned down, and internal organs and CNS were removed carefully with forceps (Qin et al., 2005).

\subsubsection{Immunocytochemical staining of NMJs}

After dissection, NMJs were fixed with $4 \%$ paraformaldehyde for $5 \mathrm{~min}$. Preparations were blocked with $950 \mu \mathrm{PBT}(0.1 \mathrm{M}$ Phosphate Buffer with $0.05 \%$ Triton X-100) mixed with $50 \mu 1$ NGS (Normal Goat Serum; 5\% solution) for 30 mins. Primary antibodies (mouse $\alpha$-Synuclein at 1:100 dilution and Rabbit- $\alpha$-Glu RIID at 1: 500 dilution in NGS blocking solution) were added and incubated over night at $4^{0} \mathrm{C}$. After incubating with primary antibodies, preparations were washed three times, 10 min each, with PBT. Secondary antibodies (anti-mouse Alexa 488 (1:500 dilution), anti-Rabbit Cy3 (1:500 dilution) and anti-HRP (Horse Radish Peroxidase) Cy5 (1:250 dilution) were added in blocking solution and incubated for $4 \mathrm{~h}$ at room temperature. After three washes with PBT (10 min each), preparations were mounted with vectashield mounting medium (Vector Laboratories, Burlingame, USA).

\subsubsection{Confocal microscopy}

Preparations stained with fluorescently tagged antibodies were used as samples to acquire images. Confocal images were taken with a Leica TCS SP5 confocal microscope (Leica Microsystems GmbH, Mannheim, Germany) using a 40 X, 1.4 numerical aperture HCX PL APO objective involving the Leica Application Suite 
Advanced Fluorescence (LAS AF) 1.5.1 software (Leica Microsystems GmbH, Mannheim, Germany).

Table 2.1: List of primary antibodies used in this study

\begin{tabular}{|c|c|c|c|c|}
\hline Antibody (Ab) & Animal & Source & $\begin{array}{l}\text { Epitope } \\
\text { detected }\end{array}$ & Dilution \\
\hline $\begin{array}{l}\text { Tyrosine } \\
\text { Hydroxylase }\end{array}$ & Rabbit & $\begin{array}{l}\text { Chemicon } \\
\text { International }\end{array}$ & $\begin{array}{l}\text { Tyrosine } \\
\text { Hydroxylase }\end{array}$ & $1: 150$ \\
\hline $\begin{array}{l}\text { Monoclonal } \alpha \mathrm{S} \\
\text { (LB509 clone) }\end{array}$ & Mouse & $\begin{array}{l}\text { Zymed } \\
\text { Laboratories }\end{array}$ & $\alpha$-Synuclein & $1: 100$ \\
\hline Polyclonal $\alpha \mathrm{S}$ & Rabbit & Anaspec Inc. & $\alpha$-Synuclein & $1: 300$ \\
\hline A11 & Rabbit & $\begin{array}{l}\text { Invitrogen } \\
\text { Corporation }\end{array}$ & $\begin{array}{l}\text { Common } \\
\text { conformation } \\
\text { of amyloid } \\
\text { oligomers }\end{array}$ & $1: 200$ \\
\hline $\begin{array}{l}\text { Monoclonal } \alpha- \\
\text { Tubulin }\end{array}$ & Mouse & Sigma Inc. & $\alpha$-Synuclein & $1: 2000$ \\
\hline Elav & Mouse & $\begin{array}{l}\text { Iowa Hybridoma } \\
\text { Bank }\end{array}$ & Elav & $1: 200$ \\
\hline HRP-Cy5 & Goat & Dianova & $\begin{array}{l}\text { Horse Radish } \\
\text { Peroxidase }\end{array}$ & $1: 250$ \\
\hline $\begin{array}{l}\text { Drosophila } \\
\text { PDF }\end{array}$ & Mouse & $\begin{array}{l}\text { Iowa Hybridoma } \\
\text { Bank }\end{array}$ & $\begin{array}{l}\text { Pigment } \\
\text { Dispersion } \\
\text { Factor }\end{array}$ & $1: 150$ \\
\hline nc82 & Mouse & $\begin{array}{l}\text { Iowa Hybridoma } \\
\text { Bank }\end{array}$ & Bruchpilot & $1: 200$ \\
\hline
\end{tabular}

Table 2.2: List of secondary antibodies used in this study

\begin{tabular}{lllll}
\hline Epitope detected & Animal & \multicolumn{1}{c}{ Source } & Label & Dilution \\
\hline Mouse IgG & Goat & Molecular Probes & Alexa 488 & $1: 500$ \\
Mouse IgG & Goat & Molecular Probes & Alexa 568 & $1: 500$ \\
Mouse IgG & Goat & Pierce & HRP & $1: 2000$ \\
Rabbit IgG & Goat & Molecular Probes & Alexa 488 & $1: 500$ \\
Rabbit IgG & Goat & Molecular Probes & Alexa 568 & $1: 500$ \\
Rabbit IgG & Goat & Pierce & HRP & $1: 2000$ \\
\hline
\end{tabular}


Table 2.3:List of fly stocks used and generated in this study

\begin{tabular}{|c|c|c|}
\hline Name & Genotype & Reference/Source \\
\hline \multicolumn{3}{|l|}{ Wildtype lines } \\
\hline white & $w^{1118}$ & $\begin{array}{l}\text { Lindsley and Zimm, } \\
1992\end{array}$ \\
\hline OreR & wildtype & $\begin{array}{l}\text { Lindsley and Zimm, } \\
1992\end{array}$ \\
\hline \multicolumn{3}{|c|}{ Gal4 driver and UAS lines } \\
\hline elav-Gal4 & $w^{*} P\{G a w B\} e l a v^{c 155}$ & $\begin{array}{l}\text { Bloomington Stock } \\
\text { number } 458\end{array}$ \\
\hline Ddc-Gal4 & $w^{*} ; ; P\{D d c-G A L 4 . L\} 4.36$ & $\begin{array}{l}\text { Bloomington Stock } \\
\text { number } 7009\end{array}$ \\
\hline TH-Gal4 & $w^{*} ; ; P\{T H-G A L 4 . F\} 3$ & Andretic et al., 2005 \\
\hline OK6-Gal4 & $w^{*} ; P\{G a w B\} O K 6$ & $\begin{array}{l}\text { S.Sigrist's Lab, FU } \\
\text { Berlin }\end{array}$ \\
\hline attP-Landing site & $\begin{array}{l}y^{l} M\left\{\text { vas-int.Dm\}ZH- } 2 A w^{*}\right. \\
M\{3 x P 3-R F P . a t t P\} Z \mathrm{ZH}-86 \mathrm{Fb}\end{array}$ & Bischof et al., 2007 \\
\hline UAS-lacZ & $\begin{array}{l}y^{l} M\{\text { vas-int.Dm }\} Z H-2 A w^{*} ; \\
M\{3 x P 3-R F P . U A S-l a c Z\} Z \mathrm{H}-86 \mathrm{Fb}\end{array}$ & $\begin{array}{l}\text { Generated in this } \\
\text { study }\end{array}$ \\
\hline UAS-EGFP & $\begin{array}{l}y^{l} M\{\text { vas-int.Dm }\} Z H-2 A w^{*} ; \\
M\{3 x P 3-R F P . U A S-e g f p\} Z \mathrm{Z}-86 \mathrm{Fb}\end{array}$ & $\begin{array}{l}\text { Generated in this } \\
\text { study }\end{array}$ \\
\hline UAS-[WT- $\alpha \mathrm{S}]$ & $\begin{array}{l}y^{l} M\{\text { vas-int.Dm }\} Z H-2 A w^{*} \\
M\{3 x P 3-R F P . U A S-[W T-\alpha S]\} Z \mathrm{Z}- \\
86 \mathrm{Fb}\end{array}$ & $\begin{array}{l}\text { Generated in this } \\
\text { study }\end{array}$ \\
\hline UAS-[TP- $\alpha S]$ & $\begin{array}{l}y^{l} M\left\{\text { vas-int.Dm\}ZH- } 2 A w^{*}\right. \\
M\{3 x P 3-R F P . U A S-[T P-\alpha S]\} \mathrm{ZH}- \\
86 \mathrm{Fb}\end{array}$ & $\begin{array}{l}\text { Generated in this } \\
\text { study }\end{array}$ \\
\hline UAS-[A56P- $\alpha \mathrm{S}]$ & $\begin{array}{l}y^{l} M\left\{\text { vas-int.Dm\}ZH-2A } w^{*}\right. \\
M\{3 x P 3-R F P . U A S-[A 56 P- \\
\alpha S]\} Z \mathrm{Z}-86 \mathrm{Fb}\end{array}$ & $\begin{array}{l}\text { Generated in this } \\
\text { study }\end{array}$ \\
\hline UAS-[A53T- $\alpha$ S] & $\begin{array}{l}y^{l} M\left\{\text { vas-int.Dm\}ZH- } 2 A w^{*} ;\right. \\
M\{3 x P 3-R F P . U A S-[A 53 T-\alpha S]\} Z \mathrm{Z}- \\
86 \mathrm{Fb}\end{array}$ & $\begin{array}{l}\text { Generated in this } \\
\text { study }\end{array}$ \\
\hline UAS-EKO/Kir2.1 & $y^{l} w^{*} ; E 222^{+}[U A S-E K O]$ & $\begin{array}{l}\text { Benjamin White } \\
\text { (NIH, USA) }\end{array}$ \\
\hline UAS-NaChBac & $y^{l} w^{*} ; U A S-N a C h B a c-G F P$ & $\begin{array}{l}\text { Benjamin White } \\
\text { (NIH,USA) }\end{array}$ \\
\hline
\end{tabular}




\subsection{Behavioral Assays}

\subsubsection{Climbing assay}

Flies expressing different $\alpha \mathrm{S}$ variants were placed in a newly designed behavior assay apparatus containing a bottom vial and an inverted upper vial. They were assayed for their ability to climb from the bottom to upper vial in thirty seconds. To avoid photic outside effects during the assay period, both vials were encased in black boxes. As flies get attracted towards the light, I have also provided a light source at the top of the upper vial by two light emitting diodes. This type of set up provides a directionality and motivation for the flies to climb from bottom to the top. A photograph of the apparatus is shown in Figure 11 A.

\subsubsection{Longevity assay}

Flies expressing $\alpha \mathrm{S}$ variants and control animals expressing lac $Z$ were collected and maintained under $12 \mathrm{~h}$ light/12h dark (LD) at $25{ }^{\circ} \mathrm{C}$ with constant humidity and population density per vial. Flies were transferred to fresh food vials and scored for survival in 5-day intervals. Survival curves were calculated and plotted using Kaplan-Meier statistics (Fall et al., 2003; Rajput et al., 2009), and differences were analysed by using the log-rank method (GraphPad Prism software, San Diego, USA).

\subsubsection{Analysis of circadian behaviour}

Flies were crossed to obtain the appropriate offspring genotype, which were allowed to develop (pre and post-eclosion stages) at $25^{\circ} \mathrm{C}$ in the $12 \mathrm{~h}$ light/12h dark (LD) conditions unless mentioned otherwise. To assess the circadian activity, flies of different genotypes were loaded into the Drosophila Activity Monitoring (DAM) systems (Trikinetics, Waltham, USA) and their activity was recorded. Initially, the flies were allowed to continue their entrainment under LD (12:12) conditions for the at least three days before they were switched into a 'free-run' mode under the $12 \mathrm{~h}$ dark/12h dark (DD) conditions using the Trikinetics data collection system. Data were analysed with Clock Lab to extract the circadian behaviour (http://www.actimetrics.com/ClockLab/) of the flies. Double-plotted 
actograms, Chi-squared or Lomb-Scragle periodograms were plotted using MATLAB 7.4 (R 2007a) software using the Clock Lab programs.

\subsubsection{Sleep assays}

Flies were grown under LD $12 \mathrm{~h}: 12 \mathrm{~h}$ at $25{ }^{\circ} \mathrm{C}$ before and after the eclosion. Males were collected from the progeny and aged with equal population density under LD 12:12 at $25{ }^{\circ} \mathrm{C}$. After 28-30 days, locomotor activity of the aged flies was recorded in LD by the Drosophila Activity Monitoring (DAM) system (Trikinetics, Waltham, USA) as described (Joiner et al., 2006; Pitman et al., 2006). "Sleep" was measured as bouts of 5 min of inactivity (Shaw et al., 2000), using a moving window of 1 min intervals. Average bout length (ABL) was calculated from the sum of sleep bouts of all lengths (in minutes) divided by the total number of sleep bouts. Algorithms written for computing these parameters are included in the appendix A. 
"WE SEE ONLY WHAT WE KNOW."

(Johann Wolfgang von Goethe)

\section{RESULTS}

\subsection{Structure-based rational design of the $\alpha$-synuclein variants}

$\alpha$-Synuclein $(\alpha S)$ is a natively unstructured protein. It has a high propensity to misfold and aggregate into fibrils in the process of amyloidogenesis (Cookson, 2005). Formation of pre-fibrillar soluble oligomers is one of the earlier steps in the aggregation pathway of $\alpha \mathrm{S}$ (Cookson, 2005). There is accumulating evidence that the small and diffusible pre-fibrillar oligomers of amyloidogenic proteins are potential pathogenic species in neurodegenerative processes, e.g. oligomers of soluble amyloid- $\beta$-protein $(A \beta)$ in Alzheimer's disease (reviewed in Haass and Selkoe, 2007) and oligomers of polyglutamine in the expanded polyglutamine disorders (Sanchez et al., 2003).

$\alpha S$ can not form stable and enriched oligomers like $A \beta$ and other amyloidogenic proteins (Lashuel and Lansbury, 2006), and aggregation is a dynamic biophysical and biochemical process. Therefore, investigating the role of soluble pre-fibrillar $\alpha \mathrm{S}$ oligomers in PD pathogenesis and understanding the nature of toxic $\alpha \mathrm{S}$ species is a challenging problem. To address this problem, we have rationally designed different variants of $\alpha \mathrm{S}$ that could potentially form more pre-fibrillar oligomeric species (in collaboration with P. Karpinar; Christian Griesinger's and Markus Zweckstetter's Lab). The rational design was based on the structural information of $\alpha \mathrm{S}$ monomer in solution and the topology of $\alpha \mathrm{S}$ 
fibrils (Bertoncini et al., 2005; Heise et al., 2005; Vilar et al., 2008). Fundamental premise in the rational design of $\alpha \mathrm{S}$ variants was preventing the fibril formation, by impinging for example on the 'cross- $\beta$ ' structure and promoting thereby the pre-fibrillar oligomer formation. Cross- $\beta$ structure in fibrils is stabilized by the extensive structural interactions of inter and intra-molecular $\beta$ strands of $\alpha \mathrm{S}$. Thus, replacement of Alanine to Proline, which serves as a $\beta$ strand breaker, was performed in the key regions of $\alpha \mathrm{S}$-monomer that would normally become the inner core of the 'cross- $\beta$ ' structure in the perspective fibrils. Several $\alpha \mathrm{S}$ variants were generated in this fashion by replacing the Alanines A30, A56 and A76, respectively (Figure 8A), and both their in vitro aggregation properties and oligomer-forming tendencies were examined by various biophysical methods as reported (Karpinar et al., 2009). The following $\alpha \mathrm{S}$ variants were generated: A30P, A56P, A76P, A30P/A56P, A30P/A76P and A30P/A56P/A76P (TP- $\alpha$ S).

\section{(on the next page)}

Figure 8: $\alpha$-Synuclein variants with reduced fibrillation propensity and impaired $\beta$ structure form more on-pathway pre-fibrillar oligomers

(A) Domain structure of $\alpha \mathrm{S}$ depicting the location of mutations in monomer and their relative position in the fibrillar structure derived from solid state NMR. These $\alpha \mathrm{S}$ variants were rationally designed to promote formation of oligomeric pre-fibrillar species by actively impairing the formation of $\beta$-structure in the fibrils resulting from aggregation. Highly ordered cross- $\beta$-structure is a paramount structural feature of the amyloidogenic fibrils. (B) Fibril formation kinetics of wildtype- $\alpha \mathrm{S} / \mathrm{WT}-\alpha \mathrm{S}$ (black), A56P- $\alpha \mathrm{S}$ (Yellow), and $\mathrm{TP}-\alpha \mathrm{S}$ (red) followed by Thioflavin T fluorescence (ThT); a.u. = arbitrary units. ThT fluorescence increases with the rate of fibril formation. (C) Dot blot assay on the nitrocellulose membrane using conformation specific A11 antibody. A11 recognizes a mixture of oligomers and monomers of TP- $\alpha \mathrm{S}(\mathrm{O} / \mathrm{M})$ but not monomeric TP- $\alpha \mathrm{S}(\mathrm{M})$ on membrane, whereas anti- $\alpha \mathrm{S}$ antibody recognizes both TP- $\alpha \mathrm{S}(\mathrm{M})$ and TP- $\alpha \mathrm{S}(\mathrm{O} / \mathrm{M})$. Results in B \& C were obtained in collaboration with Pinar Karpinar (Christian Griesinger's and Markus Zweckstetter's Lab). 
A

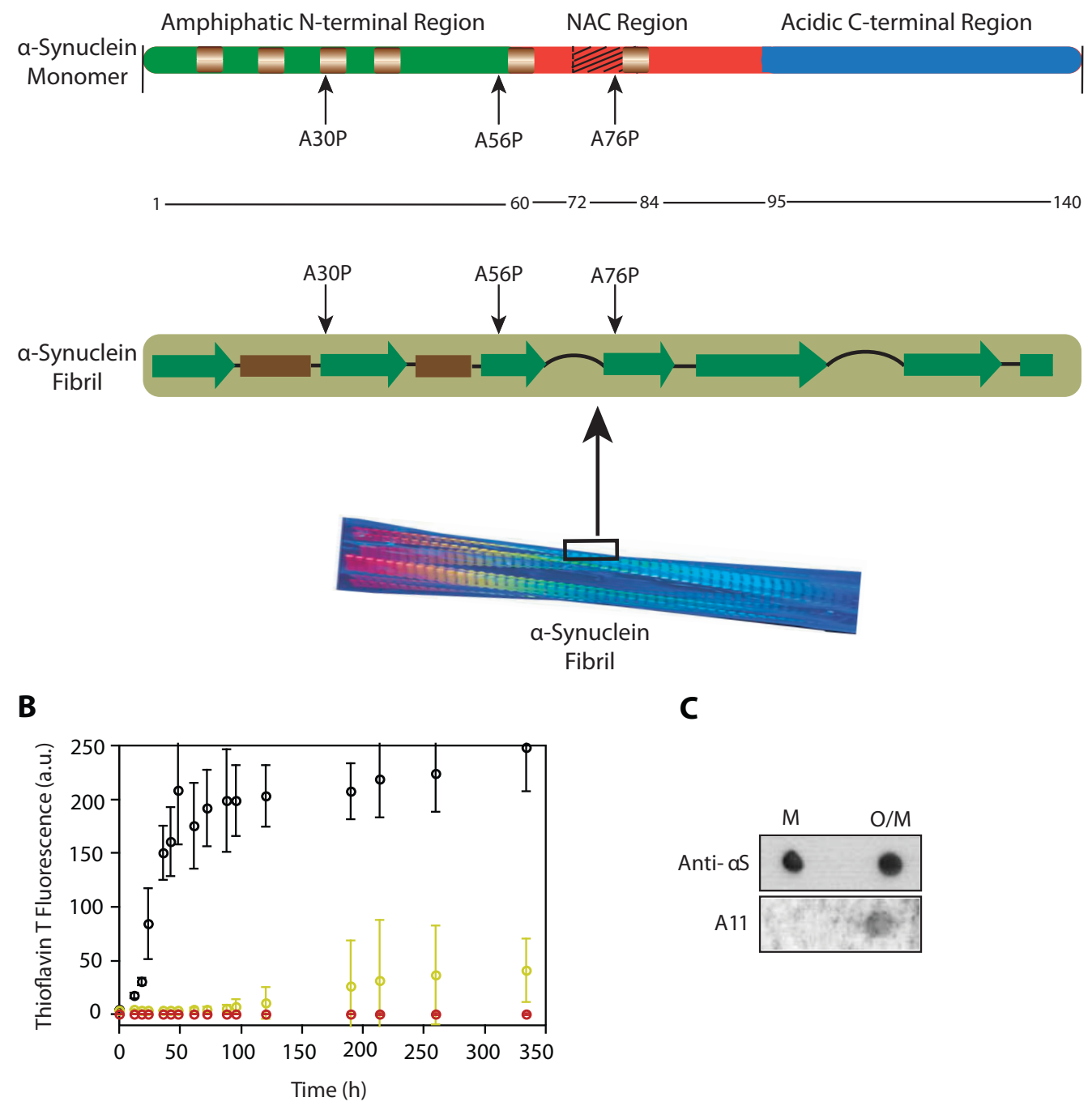




\subsection{TP- $\alpha \mathrm{S}$ and A56P- $\alpha \mathrm{S}$ show increased propensity to form oligomers and delayed fibril-formation kinetics}

Among the different $\alpha \mathrm{S}$ variants generated, A56P- $\alpha \mathrm{S}$ and TP- $\alpha \mathrm{S}$ (A30P/A56P/A76P- $\alpha$ S) variants showed a potential delay in fibril formation and an increased propensity to form oligomers (Figure 8B, C; see Karpinar et al., 2009). Soluble pre-fibrillar oligomers formed by TP- $\alpha \mathrm{S}$ and $\mathrm{A} 56 \mathrm{P}-\alpha \mathrm{S}$ also share significant structural and conformational similarity with the 'on pathway' oligomers formed by the (wild type) WT- $\alpha \mathrm{S}$. For example, liquid-state NMR and circular dichroism (CD) spectroscopy indicated that point mutations did not markedly alter the structural properties of the $\alpha \mathrm{S}$ monomer in solution; and also mutations didn't prohibit the formation of $\alpha$-helical conformation on the surface of SDS micelles (experiments were done in collaboration with the Dept. of NMR based structural biology, MPIbpc). Furthermore, conformational specific antibody, called A11, recognizes the TP- $\alpha \mathrm{S}$ oligomers (Figure $8 \mathrm{C}$ ). The A11 antibody is shown to recognize a common unknown conformation shared by toxic amyloid protein oligomers. A11 also recognizes the WT- $\alpha \mathrm{S}$ oligomers (Kayed et al., 2003) and a variety of other toxic amyloid oligomers (amyloid- $\beta$, polyglutamine, prion peptide 106-126, islet amyloid polypeptide, lysozyme).

In addition, biophysical experiments including Electron Microscopy, Atomic Force Microscopy, Dynamic Light Scattering, and Ultra Violet absorption spectrum were carried out in collaboration with M. Zweckstetter's the group as reported (Karpinar et al., 2009), and they conclusively show that both $\alpha \mathrm{S}$ structural variants, TP- $\alpha \mathrm{S}$ and $\mathrm{A} 56 \mathrm{P}-\alpha \mathrm{S}$, not only show delayed aggregation kinetics and increased propensity to form oligomers, but that both $\alpha \mathrm{S}$ variants also have impaired $\beta$ structures as summarized in the Figure 9. Most importantly, TP$\alpha \mathrm{S}$ did not form fibrils under the standard conditions used for Thioflavin T (ThT) aggregation assay (Figure 8B) and forms highest amount of soluble oligomers among the different $\alpha \mathrm{S}$ variants generated. 


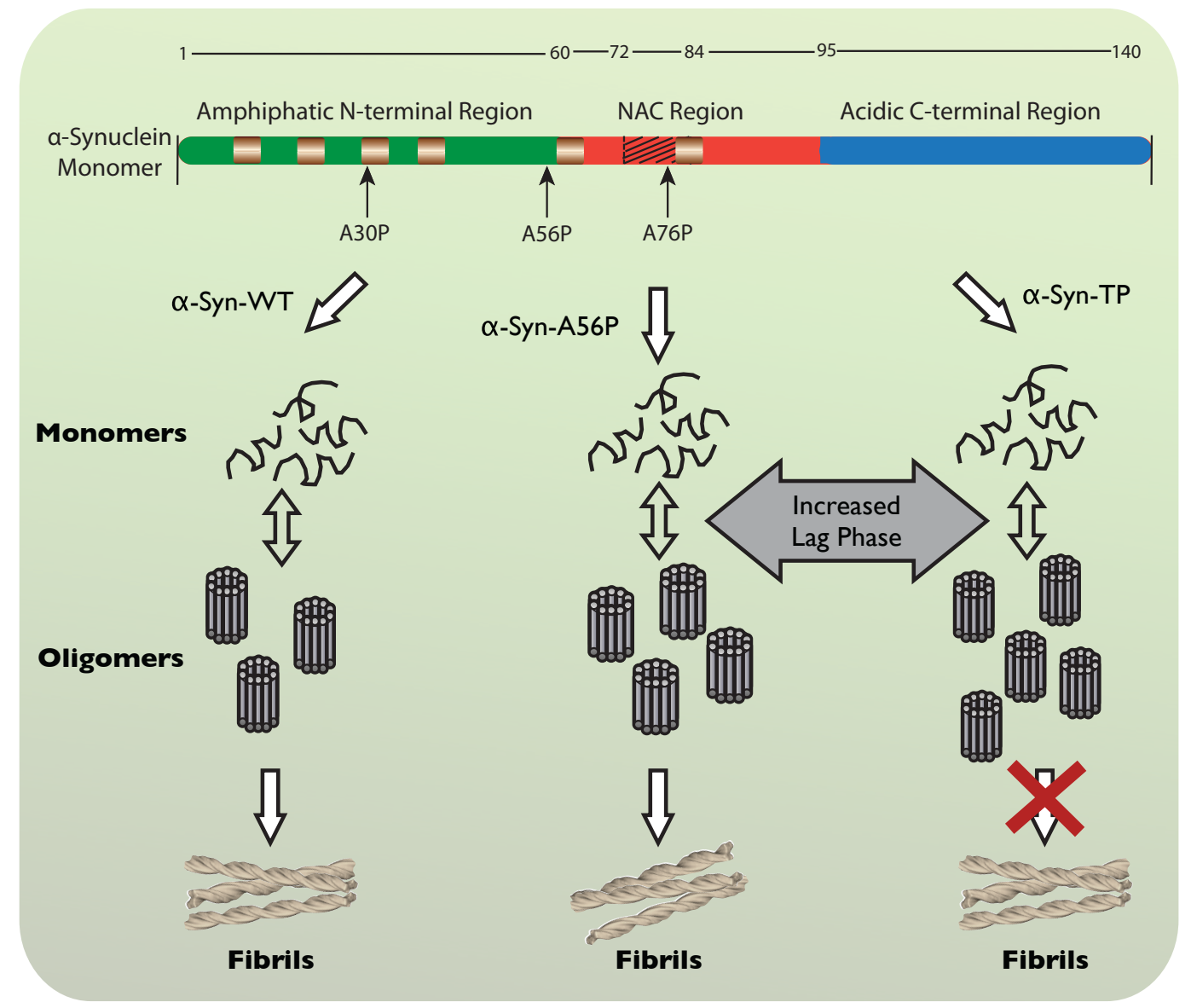

Figure 9: Illustration of the summary of results based on in vitro experiments with structure based design variants of the $\alpha \mathrm{S}$

$\alpha$-synuclein variants with impaired formation of $\beta$-structure in the perspective fibrils show delayed fibrillation kinetics with increased lag-phase, and form more of pre-fibrillar oligomers. As shown above, two important variants, A56P- $\alpha \mathrm{S}$ and TP- $\alpha \mathrm{S}$, show delayed fibril formation and increased propensity to form oligomers compared to the wildtype- $\alpha \mathrm{S}$ (WT- $\alpha S$ ). Remarkably, TP- $\alpha$ S didn't show any fibrils even after two weeks of incubation in the standard aggregation assay based on ThT fluorescence. Experimental support for this scheme derives from experiments conducted by P. Karpinar, N. Rezaei-Ghaleh, and A. Kumar of the Griesinger and Zweckstetter labs as published (Karpinar et al., 2009). 


\subsection{Generating the Drosophila model for Parkinson's disease}

My aim was to correlate the in vitro properties of structure-based $\alpha \mathrm{S}$ variants to their in vivo neurotoxicity and study its biological relevance to the $\mathrm{PD}$ pathogenesis. Studying in vivo neurotoxic effects of these structural variants of $\alpha \mathrm{S}$ helps us to better understand (i) the nature of toxic species in the aggregation pathway (intermediate pre-fibrillar oligomers or fibrils), (ii) the correlation between soluble oligomer formation and neurotoxicity, and (iii) the possible mechanism of toxicity. Therefore, to investigate the neurotoxic effects and the biological relevance of different variants of $\alpha$, I have generated a new Drosophila model for PD by using the PhiC31 based site-specific recombination system (Figure 10A). Drosophila lacks the $\alpha \mathrm{S}$ gene in its wild type genome and is a valid model for Parkinson's disease and many other neurodegenerative diseases (Auluck et al., 2002; Feany and Bender, 2000) when the $\alpha \mathrm{S}$ gene is inserted into the fly genome. In order to explore the biological consequences of $\alpha \mathrm{S}$, and $\alpha \mathrm{S}$ mutant genes, transgenic Drosophila individuals carrying the following transgenes were generated: wild type- $\alpha \mathrm{S} / \mathrm{WT}-\alpha \mathrm{S} ; \mathrm{A} 56 \mathrm{P}-\alpha \mathrm{S}$ and TP- $\alpha \mathrm{S}$ variants (the designed $\alpha \mathrm{S}$ variants, see Section 3.2); and A53T- $\alpha \mathrm{S}$, a dominant genetic mutant of $\alpha \mathrm{S}$ found in the human PD patients (Polymeropoulos et al., 1997). Using PhiC31 based site-specific recombination system for Drosophila transgenesis (Bischof et al., 2007), individual transgenic plasmid constructs, each carrying a different variant of $\alpha \mathrm{S}$ were precisely targeted to the same location in genome ("site-specific integration") which was verified by PCR using the single fly DNA PCR approach (Figure 10B; Section 2.1.10). 


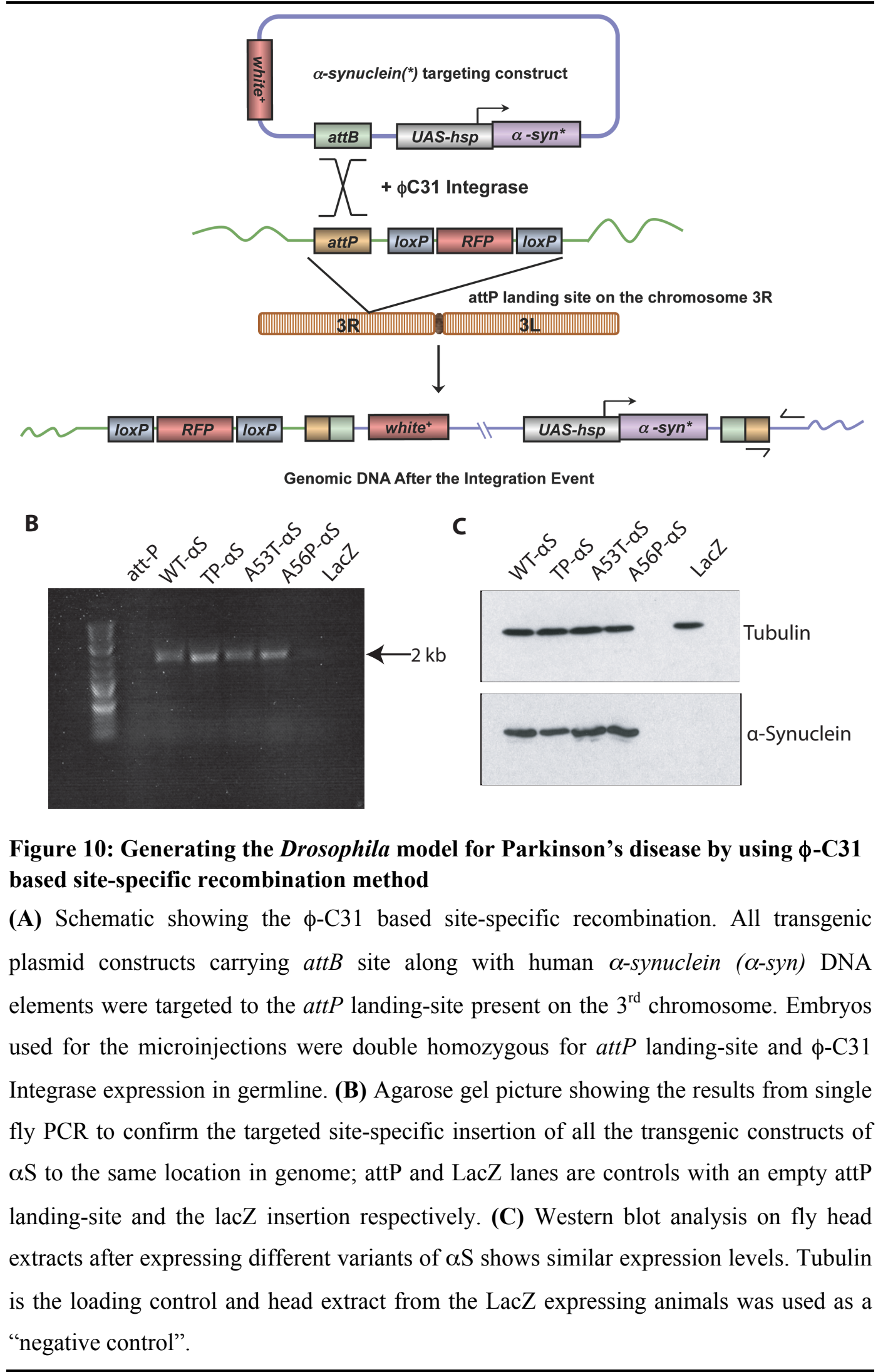


Previous results have shown that PD onset and $\alpha \mathrm{S}$ aggregation depend on the dosage and expression level of $\alpha \mathrm{S}$. The genomic duplication and triplication of $\alpha \mathrm{S}$ gene cause autosomal dominant familial Parkinson's disease (Ibanez et al., 2004; Singleton et al., 2003). Thus, another inherent advantage offered by Phi-C31 sitespecific integration has circumvented the problem of expression dosage by achieving equivalent expression levels of $\alpha \mathrm{S}$ among transgenics expressing $\alpha \mathrm{S}$ variants. To confirm this, western blot analysis done on the fly head extracts expressing different $\alpha \mathrm{S}$ variants and the results are shown in Figure 10C.

\subsection{Pre-fibrillar oligomer-enriched structural variants of $\alpha \mathrm{S}$ cause motor deficits}

PD is clinically defined as a movement disorder characterized by resting tremor, bradykinesia (slowness of movement), rigidity and postural instability, i.e. problems of initiating and stopping the movement (reviewed in Cookson, 2005). To assess whether the Drosophila PD model recapitulates the symptoms of human disorder, I have tested the motor performance of aged (4 weeks) flies expressing different variants of $\alpha \mathrm{S}$ in a climbing assay (refer to methods section 2.3.1). For their targeted expression, I used the pan-neuronal elav-Gal4 driver employing the UAS/GAL4 system (Brand and Perrimon, 1993) (Section 2.2.).

To subsequently test for fly movements in response to $\alpha \mathrm{S}$ and $\alpha \mathrm{S}$ mutant gene expression, I have designed a special climbing assay apparatus (see Figure 11A). This specially designed climbing assay apparatus is useful both to assay the negative-geotactic locomotor function and the positive-phototactic behavior of flies. In this apparatus, a light source (LED box) present at the top of upper chamber serves both as an attraction cue to motivate the flies to climb up and it provides thereby the directionality of the climbing response of the flies. The response of flies was scored as the climbing index (i.e. percentage of flies reaching the upper chamber in thirty seconds). The results summarized in Figure $11 \mathrm{~B}$ indicate that flies expressing oligomer forming TP- $\alpha \mathrm{S}, \mathrm{A} 56 \mathrm{P}-\alpha \mathrm{S}$ variants, and human associated A53T- $\alpha \mathrm{S}$ exert significant locomotor dysfunction as compared to the wildtype- $\alpha \mathrm{S} / \mathrm{WT}-\alpha \mathrm{S}$ and LacZ expressing control flies. Among 
the $\alpha \mathrm{S}$ variants examined, TP- $\alpha \mathrm{S}$ showed more chronic motor deficits as compared to the A56P- $\alpha \mathrm{S}$ and A53T- $\alpha \mathrm{S}$, respectively. This finding correlates with the ability of these $\alpha \mathrm{S}$ variants to form more pre-fibrillar oligomers in vitro (see Section 3.2). Both types of control flies, i.e. flies expressing WT- $\alpha \mathrm{S}$ and LacZ flies showed no significant locomotor dysfunction.

\section{(on the next page)}

Figure 11: Drosophila expressing pre-fibrillar oligomer enriched $\alpha$-Synuclein variants show motor deficits in the climbing assay

(A) Newly designed apparatus for the climbing assay that can be used to simultaneously assay both negative-geotactic and positive-phototactic behaviour of flies. It has a set of glass columns, each separated into lower chamber and upper chamber with a shutter in between. All the columns are encased in black vials to avoid photic effects from the outside environment. Shutters, both in the bottom and centre of all individual columns, can be opened and closed with a single switch. Light Emitting Diodes (LEDs) on the top of each column provide the light source of appropriate wavelength to serve as an attraction cue to motivate the flies to climb up. Intensity of all LEDs can be controlled using a regulator. (B) Climbing Index (percentage of flies reaching the upper chamber in a given amount of time) of four week old flies expressing different variants of $\alpha \mathrm{S}$ under the elav-Gal4 pan-neuronal driver $(\mathrm{n}=35-50$ for each genotype and values shown represent mean $\pm \mathrm{SEM} ; \mathrm{ns}=$ non-significant $P>0.05, * P<0.01, * * P<0.001)$. Significance values were determined by one-way ANOVA followed by the NewmanKeuls Multiple Comparison post hoc test. 

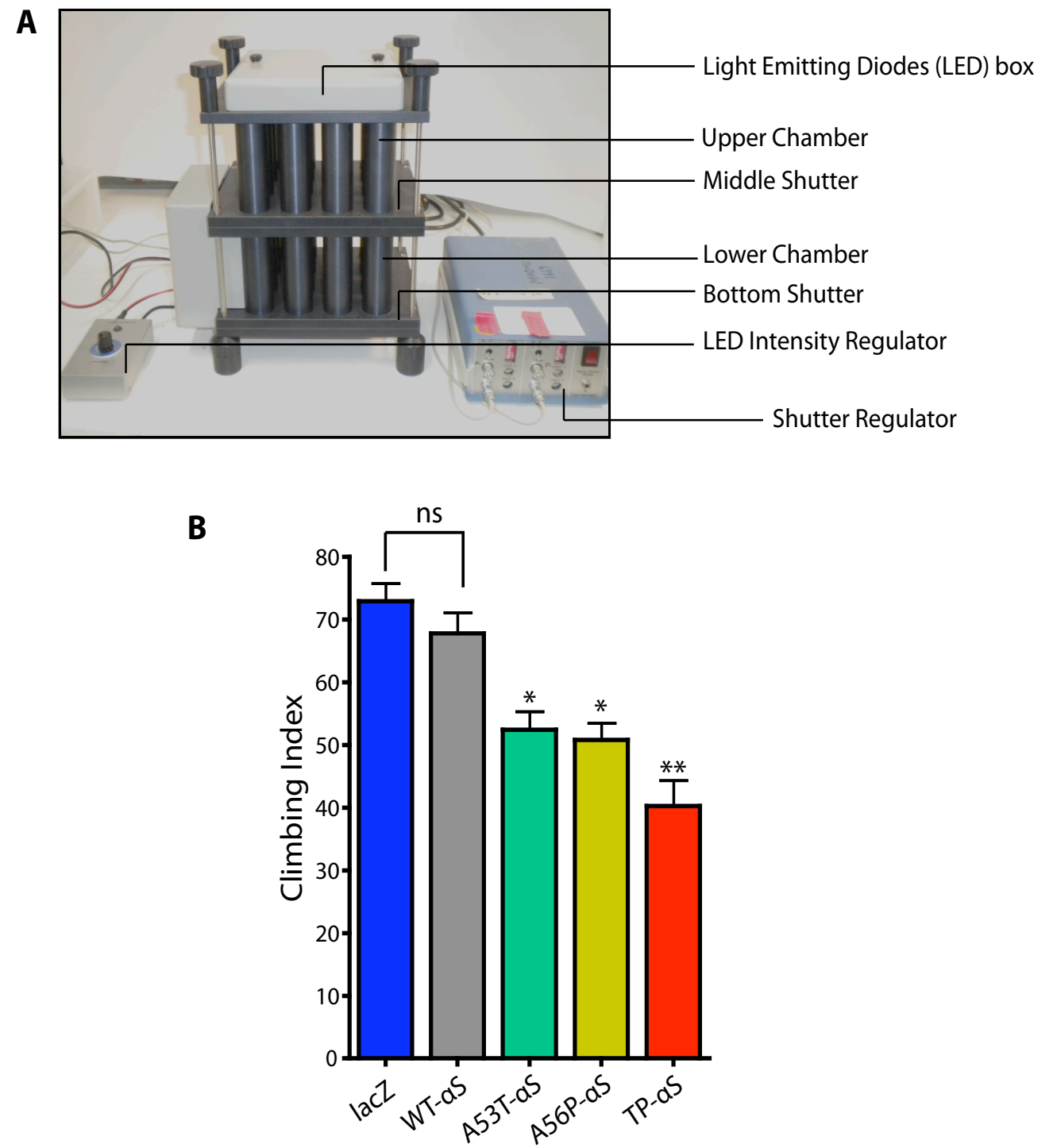

\subsection{Pre-fibrillar oligomer-enriched $\alpha S$ structural variants reduce longevity}

Parkinson's disease is not fatal, but it can reduce longevity. The disease progresses more quickly in older than in younger patients. Patients with PD have mortality rates $35 \%$ to $65 \%$ higher than those of matched controls (Fall et al., 2003; Rajput et al., 2009). To assess the effects on longevity, survival of flies expressing different $\alpha \mathrm{S}$ variants was followed over a period of 70 days. The 
results summarized in Figure $12 \mathrm{~A}, \mathrm{~B}$ show that the rate of survival has dramatically decreased after the median survival time in the flies expressing more oligomer-forming TP- $\alpha \mathrm{S}$ and $\mathrm{A} 56 \mathrm{P}-\alpha \mathrm{S}$ versions.
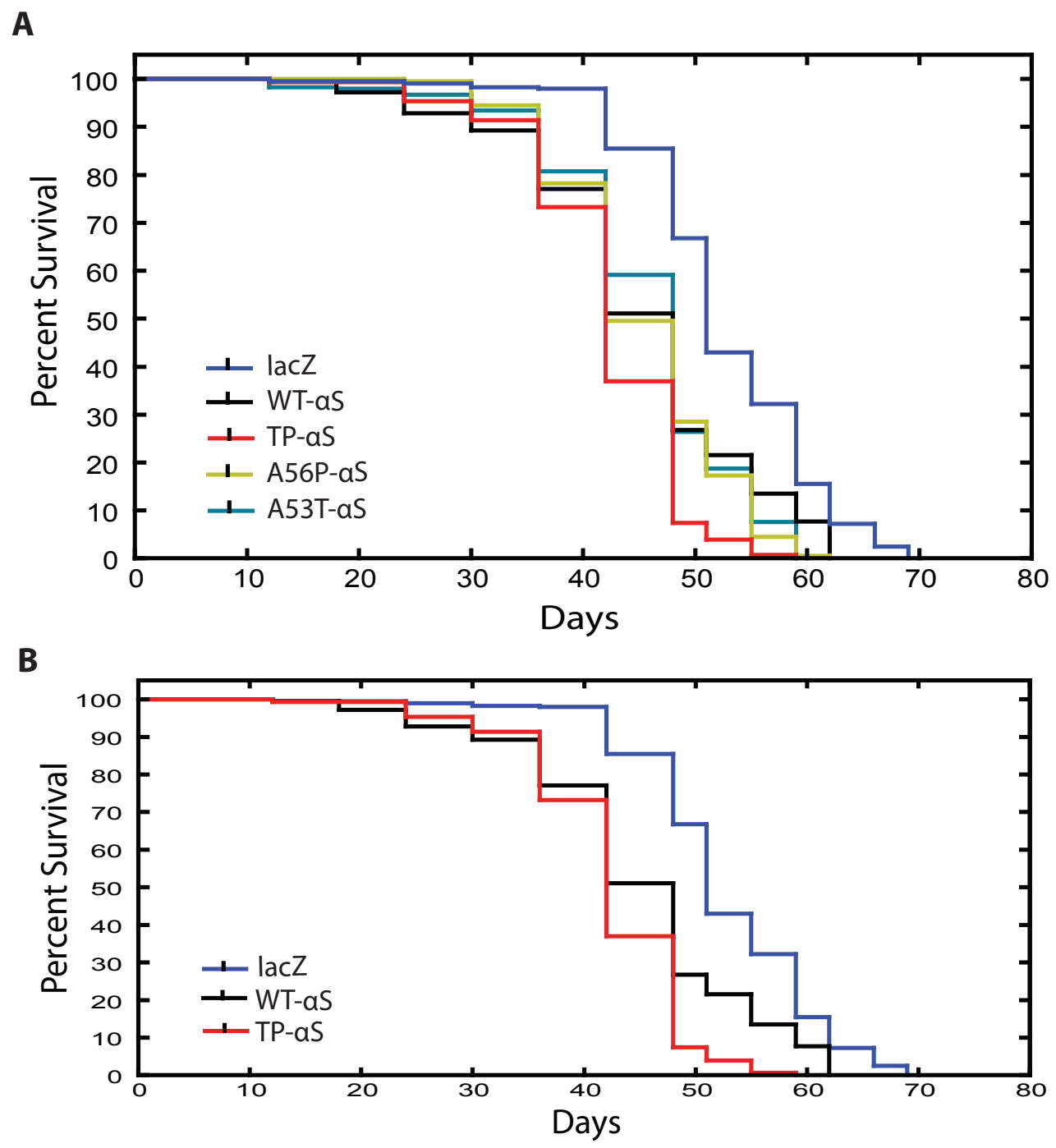

Figure 12: Longevity of flies expressing different variants of $\alpha \mathrm{S}$ and LacZ

(A-B) Kaplan-Meier survival curves of the flies expressing WT- $\alpha \mathrm{S}$ (black), TP- $\alpha \mathrm{S}$ (red), A56P- $\alpha$ S (yellow), A53T- $\alpha$ S (green), and LacZ (blue). Survival curve comparison using Logrank test shows significant differences in longevity between the WT- $\alpha$ S versus TP$\alpha \mathrm{S}(* * * P<0.0001)$, and WT- $\alpha \mathrm{S}$ versus A56P- $\alpha \mathrm{S}(* P<0.0217)$. In each experiment $\mathrm{n}=$ 350-400 for different genotypes. Note that the rate of survival has dramatically decreased after the median survival time in the animals expressing more oligomer-forming versions of the $\alpha \mathrm{S}$. 


\subsection{Expression of pre-fibrillar structural $\alpha \mathrm{S}$ variants causes age-dependent degeneration of dopaminergic (DA) neurons}

The major symptoms of PD in humans result from the profound and selective loss of DA neurons in the Substantia Nigra pars Compacta (SNc) (Braak et al., 2003). Studies on Drosophila models of PD have also reported the specific loss or dysfunction of DA neurons in the adult fly brain (Chen and Feany, 2005; Cooper et al., 2006; Feany and Bender, 2000; Periquet et al., 2007). Particularly, the dorsomedial (DM) and dorsolateral (DL) clusters of DA neurons (schematic in Figure $13 \mathrm{~A}$ ), were known to be preferentially sensitive or vulnerable to the $\alpha \mathrm{S}$ toxicity (Periquet et al., 2007; Sang et al., 2007). In addition, DM and DL clusters are easy to identify and count. Therefore, I followed age-dependent degeneration of the DA neurons in DM and DL clusters by expressing WT- $\alpha \mathrm{S}$ and the different variants of $\alpha \mathrm{S}$ (TP- $\alpha \mathrm{S}, \mathrm{A} 56 \mathrm{P}-\alpha \mathrm{S}$, and $\mathrm{A} 53 \mathrm{~T}-\alpha \mathrm{S})$ using the pan-neuronal elavGal4 driver.

\subsubsection{No degeneration of DA neurons in young flies expressing $\alpha S$ variants}

DA neurons were positively stained with the anti tyrosine-hydroxylase antibody in two-day old adult whole-mount fly brains that express different variants of $\alpha \mathrm{S}$. DM and DL clusters were separately analyzed and the number of neurons was counted as described in Section 2.2.4. As shown in the Figure 13B, C there were no significant differences in the number of DA neurons in the young flies ( 2 days after hatching) expressing different variants of $\alpha \mathrm{S}$. 
A

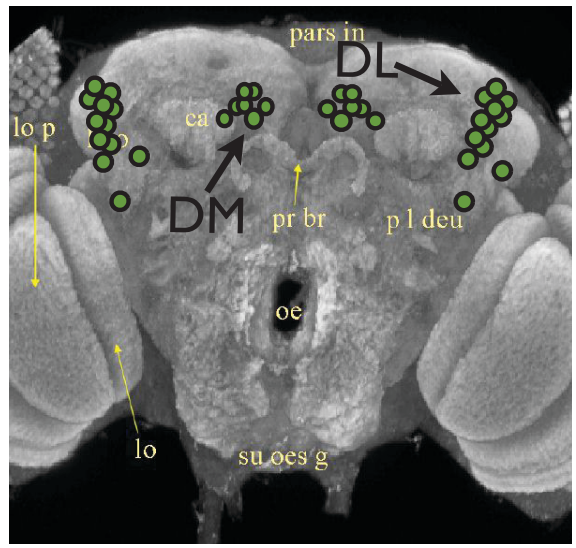

B

DL-Cluster (young)

C

DM-Cluster (young)
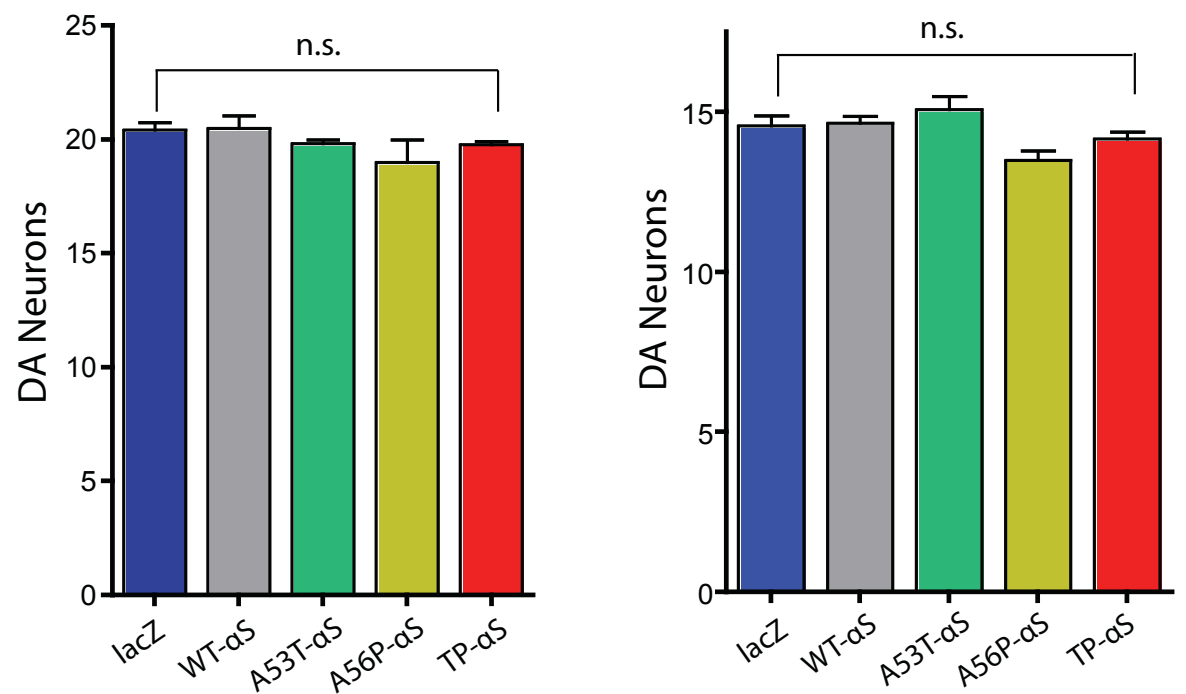

Figure 13: Dopaminergic (DA) neurons in the young flies expressing different variants of $\alpha S$ and $L a c Z$

(A) Schematic representation of the distribution of DA neurons in dorsolateral (DL) and dorsomedial (DM) clusters (black arrows) in the adult Drosophila brain as defined by Sang et al., 2007. DM and DL clusters are well-characterized, easy to count, and are known to be sensitive to the $\alpha \mathrm{S}$ neurotoxicity. (B-C) Quantification of DA neurons in the DM and DL clusters in the whole-mount young adult brains (two-day-old) expressing different $\alpha \mathrm{S}$ variants or lacZ. DA neurons were positively stained with anti-TH (antiTyrosine Hydroxylase) antibody. The values shown represent \pm SEM and $n>12$ for each genotype in all experiments; n.s. $=$ non-significant $P>0.05$. Significance values were determined by one-way ANOVA followed by the Newman-Keuls Multiple Comparison post hoc test. There are no statistically significant differences in the number of DA neurons among the 2-day old adult flies expressing different variants of $\alpha \mathrm{S}$ and LacZ. 


\subsubsection{Degeneration of DA neurons in old flies expressing $\alpha S$ variants}

In contrast to the young flies, the old flies (30 days after hatching) expressing different variants of $\alpha \mathrm{S}$ showed significant differences in the DA numbers of both DL and DM clusters as shown in Figure 14B, C. Flies expressing TP- $\alpha \mathrm{S}$ version showed more highly degenerated DM and DL clusters (Figure $14 \mathrm{D}, \mathrm{E})$ compared to the A56P- $\alpha \mathrm{S}$, which forms lesser oligomers than TP- $\alpha \mathrm{S}$. Neurotoxicity of A53T- $\alpha \mathrm{S}$ was in a similar range as observed with A56P- $\alpha \mathrm{S}$, whereas the WT- $\alpha \mathrm{S}$ and LacZ caused no significant loss of the DA neurons. The loss of neurons correlates well with the deficits observed in climbing assay and longevity assay, i.e. flies expressing more pre-fibrillar oligomer forming TP- $\alpha \mathrm{S}$ exhibited highest neurotoxicity.

(on the next page)

Figure 14: Age dependent degeneration of DA neurons in the flies expressing different variants of $\alpha S$

(A) Schematic representation of the distribution of DM and DL clusters (black arrows) of DA neurons in the adult Drosophila brain as defined by Sang et al., 2007. (B-C) Quantification of DA neurons in the DM and DL clusters in whole-mount adult brains of the 30-day-old animals expressing $\alpha \mathrm{S}$ variants and lacZ control. DA neurons were positively stained with anti-TH (anti-Tyrosine Hydroxylase) antibody. The values shown represent \pm SEM and $\mathrm{n} \geq 12$ for each genotype in all experiments; ${ }^{*} P<0.01,{ }^{* *} P<$ 0.001. Significance values were determined by one-way ANOVA followed by the Newman-Keuls Multiple Comparison post hoc test. (D-E) Representative confocal images (maximum z-projections) from the anti-TH stained (green) whole amount brains of WT- $\alpha$ S and TP- $\alpha$ S (30-day-old males). Loss of TH immunostaining/degeneration of DA neurons in the DM cluster (white arrow) of TP- $\alpha \mathrm{S}$ compared to the WT- $\alpha \mathrm{S}$ DM cluster. 
A

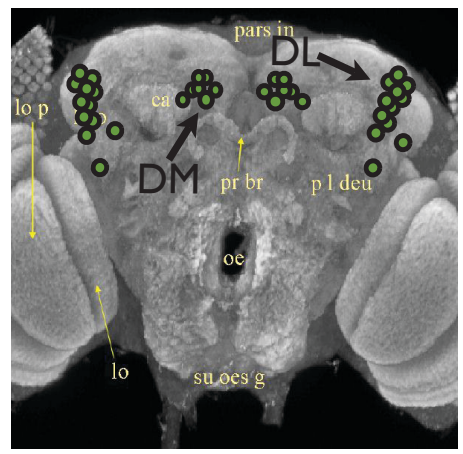

B

DL Cluster (old)

C DM Cluster (old)
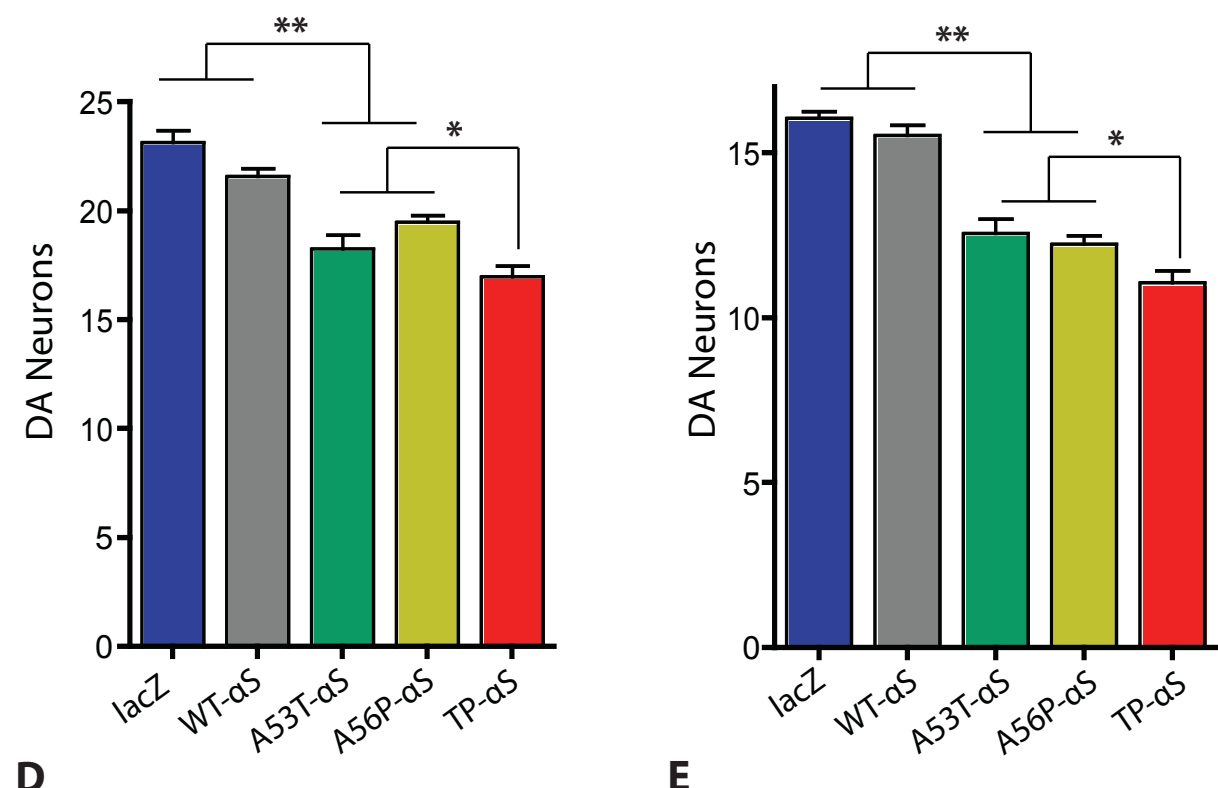

E
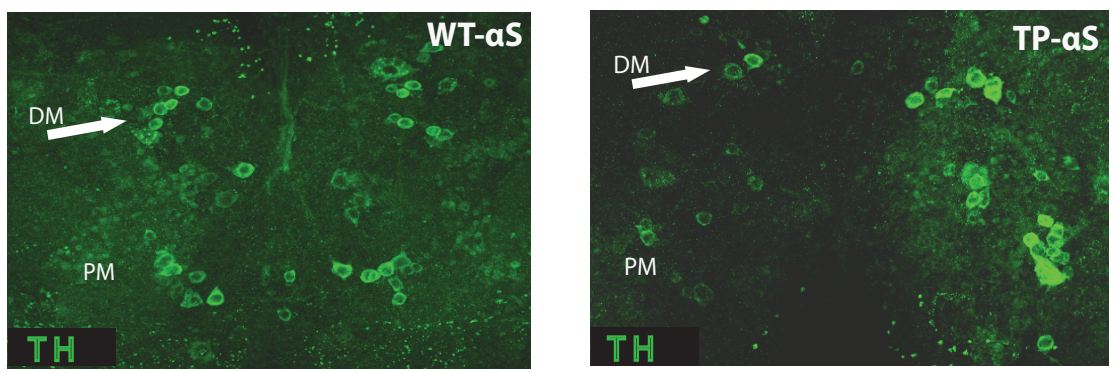


\subsection{Dopamine dependent behavioral deficits in flies expressing $\alpha \mathrm{S}$ variants}

Drosophila exhibits cardinal features of circadian rhythms and sleep (reviewed in Hendricks and Sehgal, 2004) similar to mammals (Harbison et al., 2009). In these processes, the DA neuronal circuit and signaling play evolutionarily conserved and vital roles in a wide spectrum of behavioral and molecular pathways (Shirlee Tan, 2003). In Drosophila, DA neurons were shown to have a potential role in circadian control of locomotor activity (Suh and Jackson, 2007) and in modulation of sleep-wake states (Andretic et al., 2005; Dzirasa et al., 2006; Huber et al., 2004; Kume et al., 2005) in a mammal-like manner. These features make the Drosophila PD model an attractive and valuable system to understand the etiology of non-motor symptoms of human PD including sleep disorders, deterioration of cognition, depression and disturbances of autonomic function (Chaudhuri and Schapira, 2009; Poewe, 2008). Therefore, I have studied the effect of expression of $\alpha \mathrm{S}$ variants on specific DA dependent behaviors such as sleep and circadian locomotor activity rhythms.

\subsection{Sleep abnormalities in PD flies expressing $\alpha \mathrm{S}$ variants}

Sleep abnormalities are highly prevalent in PD ( $>76 \%$ of patients) (Rye, 2006; Schapira, 2004). In order to test whether this disorder relates to DA neurons, I examined the function of WT- $\alpha \mathrm{S}$ and $\alpha \mathrm{S}$ expression in DA neurons using the Ddc-Gal4 driver. In Drosophila, the DA neurons project to the mushroom body (MB) lobes (Zhang et al., 2007) which plays a dynamic role in sleep regulation (Joiner et al., 2006; Pitman et al., 2006). In addition, DA neuronal signaling is also known to have a direct and critical effect on the regulation of sleep/wake cycle in Drosophila (Andretic et al., 2005; Kume et al., 2005). Thus, after expressing different $\alpha \mathrm{S}$ variants specifically in DA neurons, I studied the sleep and assayed various sleep parameters as outlined and detailed in Figure 15. 

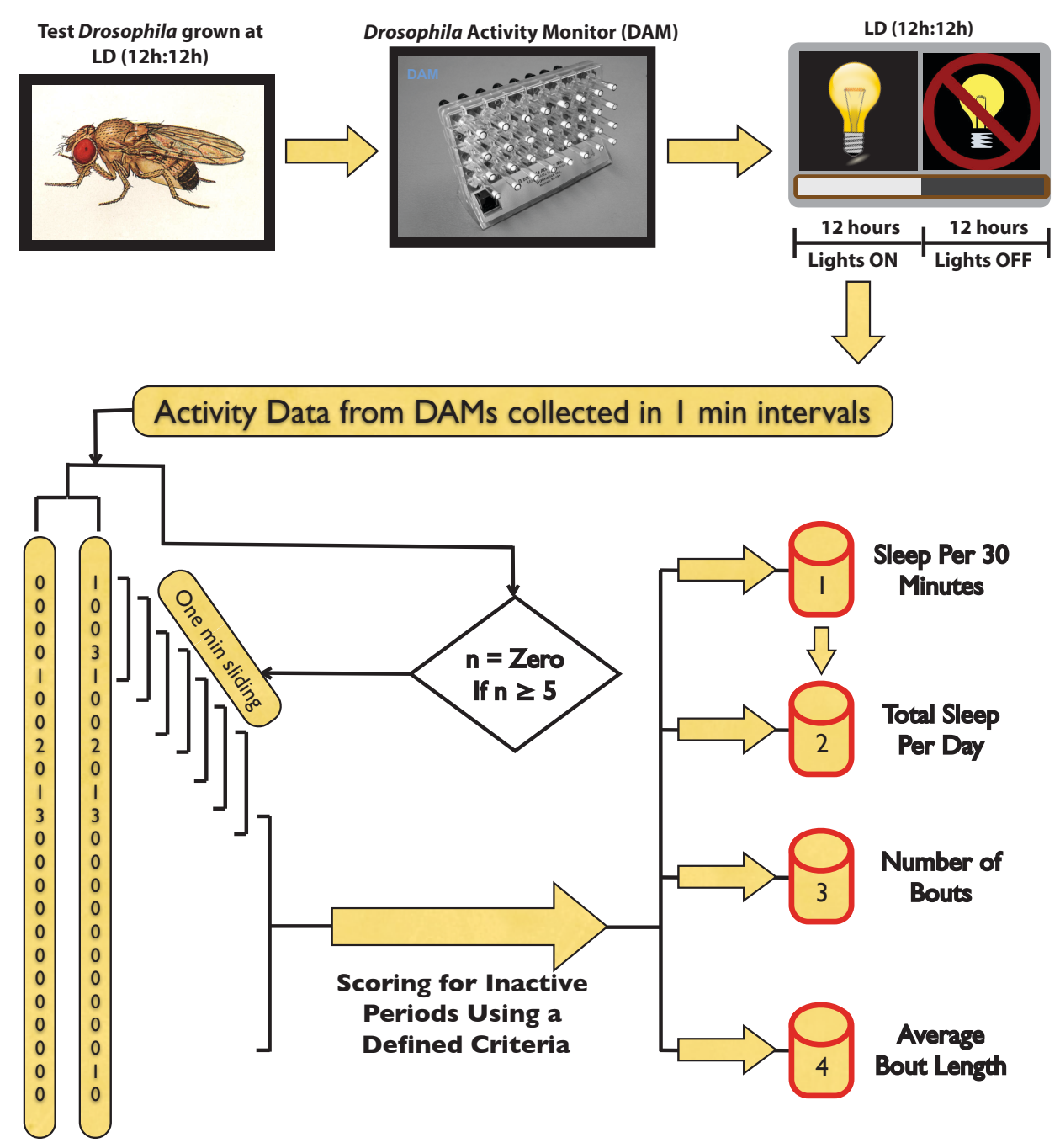

Figure 15: Flow chart depicting the sleep assay and analysis in Drosophila

Flies with different genotypes were grown at $25^{\circ} \mathrm{C}$ under LD (light $12 \mathrm{~h}$ : dark $12 \mathrm{~h}$ ) conditions. They were loaded into the Drosophila Activity Monitors (DAMs) in the first week after eclosion. Each DAM has 32 channels $(8 \times 4)$, and each channel can accommodate a tiny transparent glass tube containing a single fly. Glass tube has a 'foodcap' at one end and a stopper at the other end. DAMs monitor the activity of individual flies as they move across the glass tube, and the data from each channel resulting from a single fly can be stored and analyzed separately. For all the sleep assays, data has been collected in one-minute bins. Inactive periods of a fly are displayed as 'zero' $(0)$ in the DAM data. Sleep is defined as a bout of 5 or more minutes of inactivity (Shaw et al., 2000). Our algorithm (see appendix A) scans for the five or more consecutive zeroes with one-minute sliding window in the DAM data of individual flies and scores different sleep parameters (for e.g. total sleep, bout length, and bout number) with defined criteria. 
In Drosophila, sleep episodes are interrupted both at day and night. Sleep parameters like total amount of sleep, bout length (= average length of sleep episodes), and the bout number (= average number of sleep episodes) were measured for flies expressing $\alpha \mathrm{S}$ and different variants of it as outlined in Fig.8 (Cirelli et al., 2005; Foltenyi et al., 2007; Joiner et al., 2006; Pitman et al., 2006). Figure 16A, B show that flies expressing $\alpha \mathrm{S}$ variants exert significant differences in the quality of sleep as defined by both bout numbers and bout lengths. However, the total length of the sleep episodes of flies of the different genotypes was not affected (Figure 16C). Most importantly, the severity of the effects on sleep quality, as defined by decreased bout numbers and increased bout lengths as compared to the WT- $\alpha \mathrm{S}$ and lacZ expressing flies, corresponds to pre-fibrillar oligomer forming tendencies of the $\alpha \mathrm{S}$ variants, i.e. the flies expressing TP- $\alpha \mathrm{S}$ were stronger affected than flies expressing $\mathrm{A} 56 \mathrm{P}-\alpha \mathrm{S}$ or the $\mathrm{A} 53 \mathrm{~T}-\alpha \mathrm{S}$ variants. The observed effects only on bout numbers and lengths indicate that the quality of sleep is significantly affected. Furthermore, changes in both sleep bout numbers and bout lengths, without any effect on total sleep, indicate that the mechanisms regulating sleep initiation and sleep maintenance are affected by the expression of the mutant $\alpha \mathrm{S}$ variants. In addition, flies expressing the TP- $\alpha \mathrm{S}$ variant showed a highly abnormal sleep profile as compared to the WT- $\alpha \mathrm{S}$ and lacZ-expressing flies (Figure 16D).

To ensure that the observed sleep phenotypes are not the consequences of the indirect abnormal waking time activity, I further characterized the activity index (= activity levels during waking time) of wild type, $\alpha \mathrm{S}$ and $\alpha \mathrm{S}$ mutant flies. Activity index gives information about the activity levels of flies are when they are not sleeping, thus it helps us to know whether the effects on sleep episodes are because of the indirect abnormal wake-time activity or the direct effects on sleep behavior. Absence of any significant differences in the activity index indicates that the sleep phenotypes of the flies are not due to an abnormal wake time activity (Figure 16E). Taken together, the flies expressing oligomer-forming versions of the $\alpha \mathrm{S}$ (TP- $\alpha \mathrm{S}$ and $\mathrm{A} 56 \mathrm{P}-\alpha \mathrm{S}$ ) have seriously perturbed sleep quality 
in a way similar to the sleep problems observed in PD patients (Arnulf et al., 2008; Factor, 2002; Zimmerman, 2008; Harbison, 2009). The severity of the effects on sleep is directly correlated with the tendency of the mutant $\alpha \mathrm{S}$ variants to form oligomers in vitro. Thus, the effects on Drosophila sleep not only consolidate the pattern of toxicity as observed with the above described experiments showing that oligomer forming $\alpha \mathrm{S}$ mutants are more toxic than wild type, but they can also serve as sensitive readout to correlate the degree of in vitro oligomer formation with toxic effects in vivo.

(on the next page)

Figure 16: Dopamine dependent sleep deficits in the flies expressing different variants of $\alpha S$

(A) Bout numbers (sleep episodes) are drastically decreased in the flies expressing the oligomer-forming versions of the $\alpha \mathrm{S}$ compared to the flies expressing WT- $\alpha \mathrm{S}$ and lacZ controls. (B) Bout length (average length of sleep episodes) in flies expressing different variants of $\alpha \mathrm{S}$ is altered in a diametrically opposite fashion to the pattern of bout numbers. (C) There are no significant differences in the total amount of sleep in the flies expressing different variants of $\alpha \mathrm{S}$. (D) Sleep profile of TP- $\alpha \mathrm{S}$ (red) is drastically altered compared to the WT- $\alpha \mathrm{S}$ and lacZ flies; light and shaded rectangles represent the light/dark (12h: 12h) condition respectively. (E) Activity index (activity levels during wake period) of the different genotypes expressing variants of $\alpha \mathrm{S}$. For all the genotypes $\mathrm{n}=32$ in all experiments, and the values represent $\pm \mathrm{SEM}$; n.s. $=$ non significant $P>0.05$, $* P<0.01, * * P<0.001$. Significance values were determined by one-way ANOVA followed by the Newman-Keuls Multiple Comparison post hoc test. 


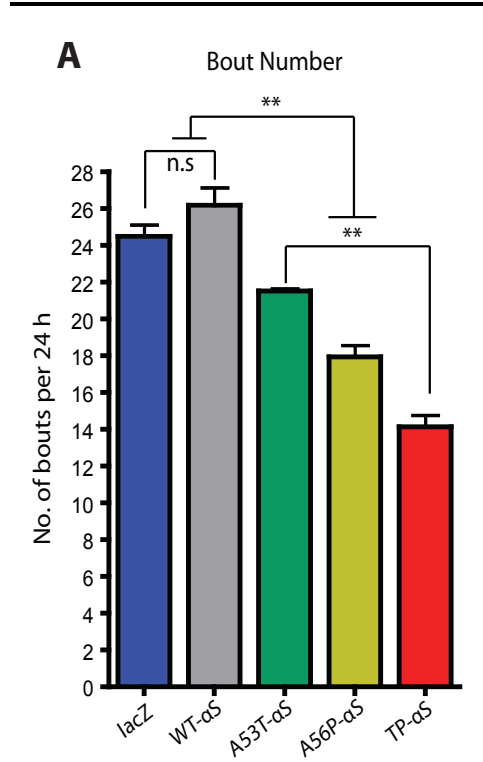

D

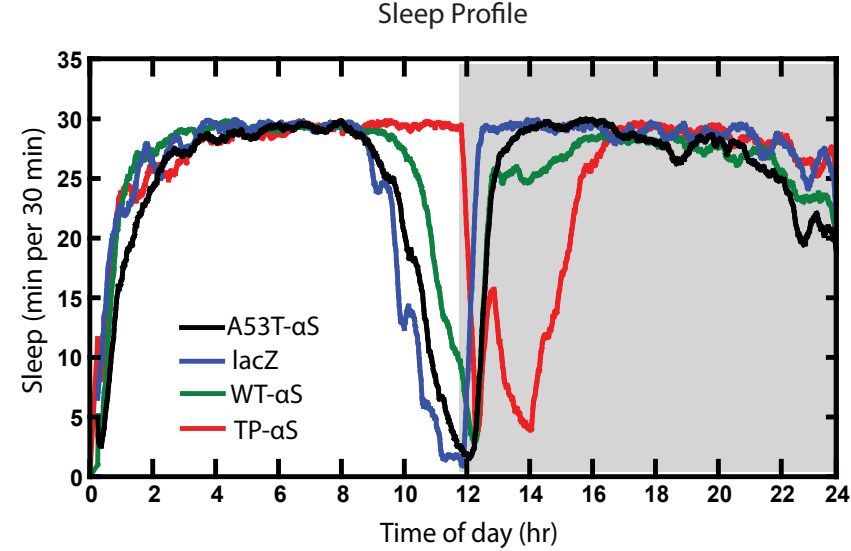

C

Total Sleep
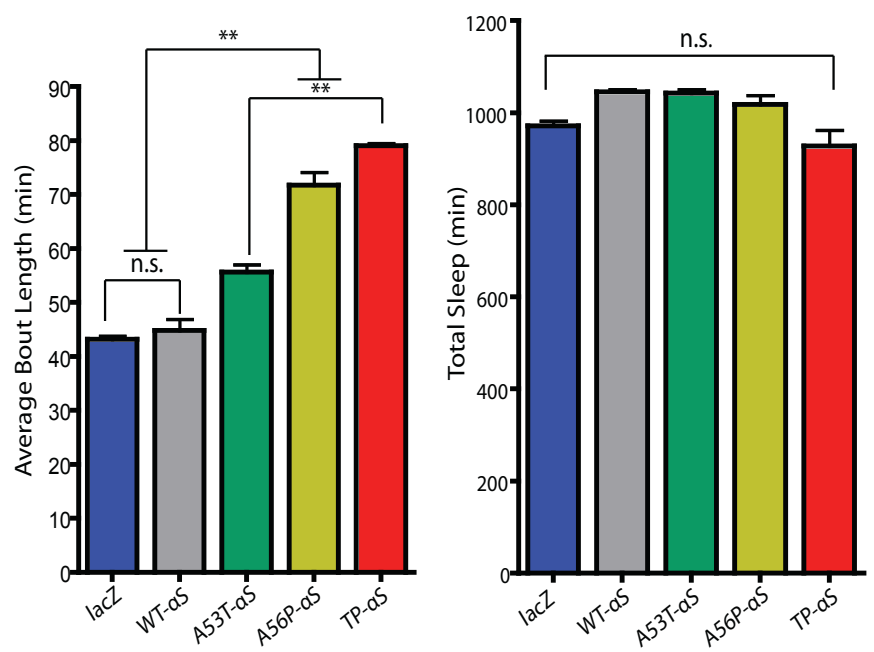

E Activity Index

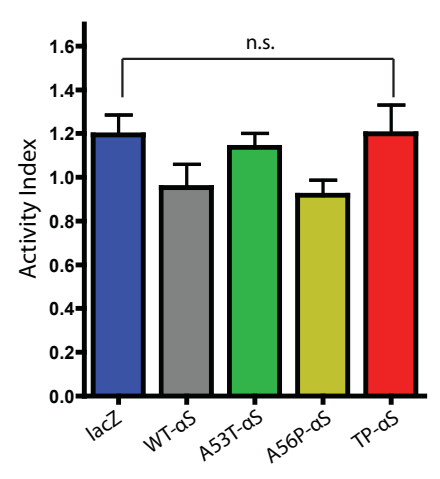

\subsection{Abnormalities in circadian locomotor activity behavior of flies expressing an oligomer-forming $\alpha \mathrm{S}$ variant}

In Drosophila, mutations and drugs that affect dopamine signaling have been shown to have an impact on a broad range of behaviors such as locomotion, learning, sleep, sexual performance, decision-making, mechanosensory startle and visual tracking (Andretic et al., 2005; Bainton et al., 2000; Kim et al., 2007; Kume et al., 2005; Ye et al., 2004). Dopaminergic neuronal signaling in Drosophila is also predicted to have a role in translating the circadian molecular oscillations in clock cells into the locomotor activity rhythms (Hirsh et al., 2010; Suh and Jackson, 2007). I examined, therefore, the circadian locomotor activity rhythms of 
flies expressing the "more-oligomer-forming variant" of $\alpha \mathrm{S}$ using the TP- $\alpha \mathrm{S}$ mutation.

Typical experiments to study circadian rhythms were conducted according to the scheme and setup depicted in Figure 17. The rhythms of circadian locomotor activity of Drosophila reflect the direct output of the central circadian clock in the brain (Rosato and Kyriacou, 2006). Rhythmic locomotor activity profiles of flies are comparable to the sinusoidal waves with period $(\mathrm{T})$, phase $(\phi)$, and amplitude (A) functions. The same parameters can therefore be used to study activity rhythms as outlined in Figure 18. In addition, another interesting feature of Drosophila circadian locomotor activity is entrainment (Figure 17A), i.e. the phenomenon that circadian clocks can 'learn' to anticipate and react to predictable external cues rather than merely respond to them. This phenomenon describes an anticipation of light transition which results in a slow increase in locomotor activity already a few hours prior the actual lights-on/off transition. It reflects the ability of the "clock" to start a certain behavior as a reaction to predictable external cues such as a lights-on/lights-off transition.

(on the next page)

\section{Figure 17: Schematic representation of a typical circadian experiment}

Flies with different genotypes (WT- $\alpha \mathrm{S}, \mathrm{TP}-\alpha \mathrm{S}, \mathrm{Lac}-\mathrm{Z}, w^{1118}$ ) were grown at $25^{\circ} \mathrm{C}$ under LD (light 12h: dark 12h) conditions. They were loaded into the Drosophila Activity Monitors (DAMs) for circadian locomotor activity monitoring. Each DAM has 32 channels $(8 \mathrm{x} 4)$ and each channel can accommodate a tiny transparent glass tube with a single fly. Glass tube has a 'food-cap' at one end and a stopper at the other end. DAMs monitor the activity of individual flies, and data from each channel resulting from a single fly can be stored and analyzed separately (as described in the Section 2.3.3). In Drosophila, locomotor activity rhythms reflect the output rhythms of central circadian clock in the brain (Rosato and Kyriacou, 2006). Therefore, the locomotor activity recorded from DAMs can used to study the circadian rhythmicity. For the sake of explanatory simplicity, I've divided the circadian activity experiment into two phases:

Phase A and B. $w^{1118}$ refers to the fly genotype. 


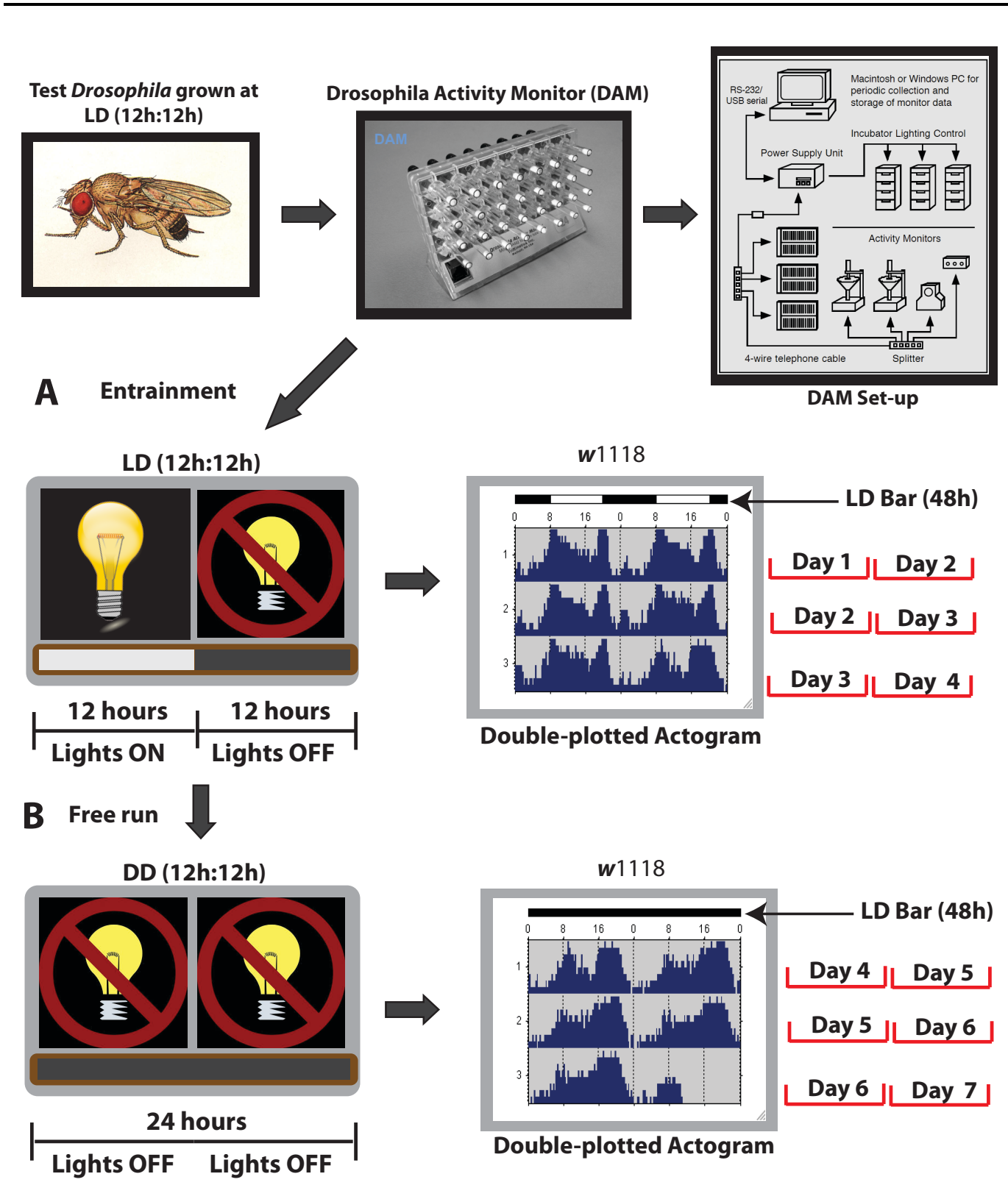

Phase A experiments reported in this work involve the following steps: 1) flies were entrained under LD (12h: 12h) conditions for 2 days after loading into DAMs, 2) experiment was continued for 3-4 more days in DAMs under the same LD (12h: 12h) conditions, and 3) the resulting data from the experiment was used for generating circadian activity profiles and double-plotted actograms (as exemplified by the $w^{1118}$ histogram above). Actograms show the activity ( $\mathrm{Y}$-axis) as a function of time (X-axis). LD bar on the top of each actogram shows the timings of lights-on/off condition with light/dark colors at corresponding time points on the X-axis. Steps covered in the Phase A are sufficient for some circadian experiments. $w^{1118}$ refers to the fly genotype. 
Phase B experiments involve an extension to the Phase A with an additional step of switching LD (12h: 12h) condition to the continuous dark (DD) condition and thereafter recording the DAM data for at least 4 days. This step is also called free-run because there won't be any external light cue given to the internal clock. Clock is allowed to run freely after the entrainment in Phase A. Circadian behavior from the DD experiment (Phase B) is useful to analyze the real functionality of internal biological clock. Data was analyzed by plotting into actograms and periodograms.
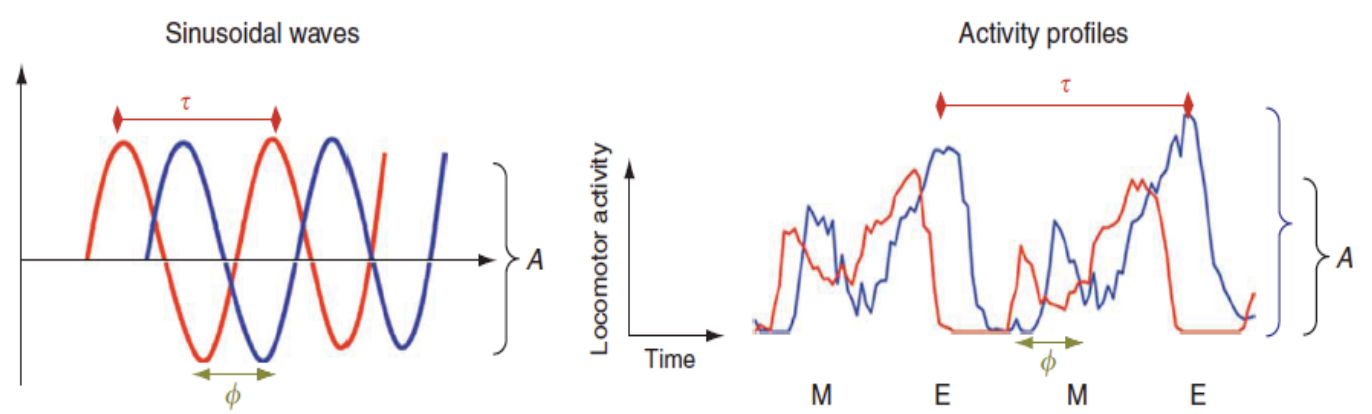

\section{Figure 18: Properties of rhythmic data}

Waves are characterized by period ( $\tau$ or T), amplitude (A) and phase $(\phi)$, illustrated in the left panel by two sinusoidal waves having a same $\tau$ and $\mathrm{A}$ but different $\phi$. Correspondingly, the same parameters can be used to describe activity profiles, right panel. The activity profiles in this figures have the same $\tau$ but different $A$ and $\phi$. They represent the average of many flies under DD conditions. $\mathrm{M}$ and $\mathrm{E}$ indicate the subjective morning and evening peaks of activity, respectively (Rosato and Kyriacou, 2006).

Since the TP- $\alpha \mathrm{S}$ mutation showed the strongest effects of the $\alpha \mathrm{S}$ variants in the behavioral assays applied, and it exerted also the highest neurotoxicity effects on DA neurons, I specifically assessed the circadian locomotor rhythms of flies expressing a Ddc-Gal4 driven TP- $\alpha \mathrm{S}$ transgene in DA neurons and compared the effects of flies expressing $\alpha \mathrm{S}-\mathrm{WT}$ and lacZ transgenes under otherwise identical conditions. As shown in Figure 19, there were no significant differences between the circadian profiles of WT- $\alpha \mathrm{S}$ and TP- $\alpha \mathrm{S}$. In particular, the anticipatory behavior remained unchanged (see the black arrows in Figure 19A, B). 


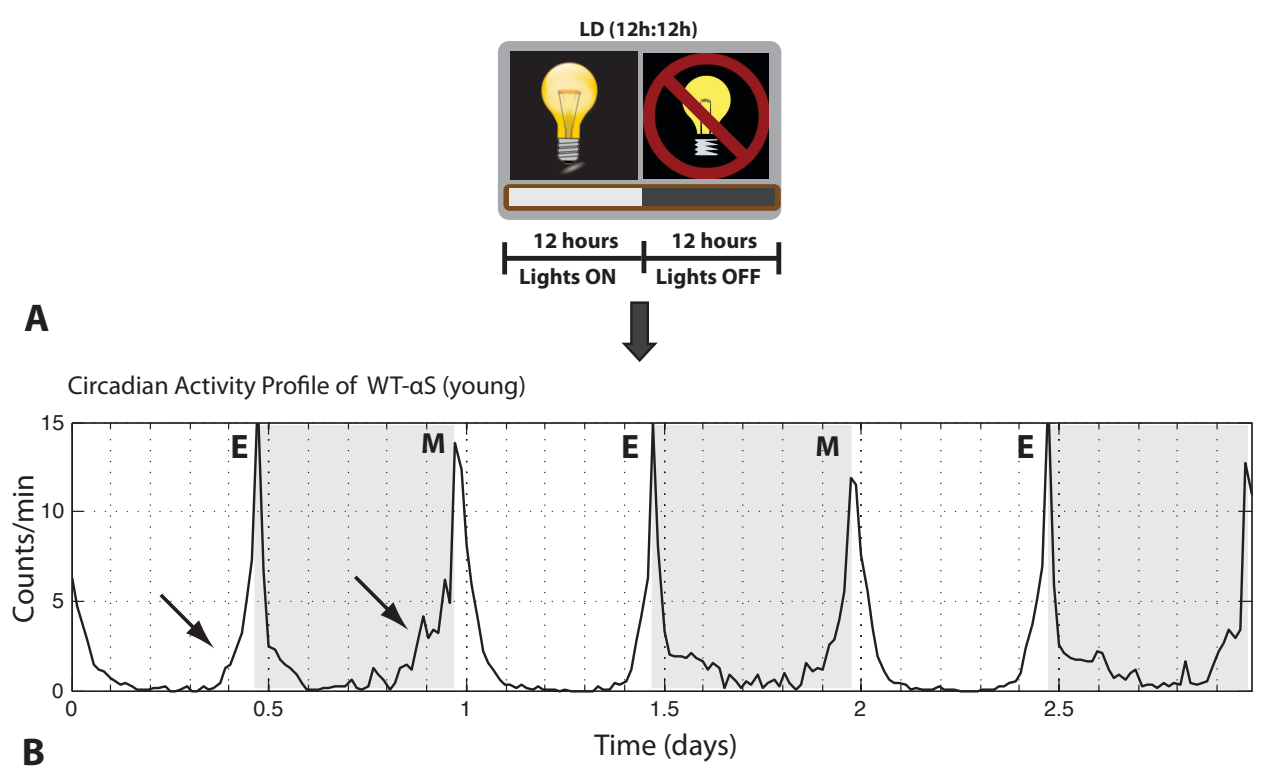

B

Circadian Activity Profile of TP-aS (young)

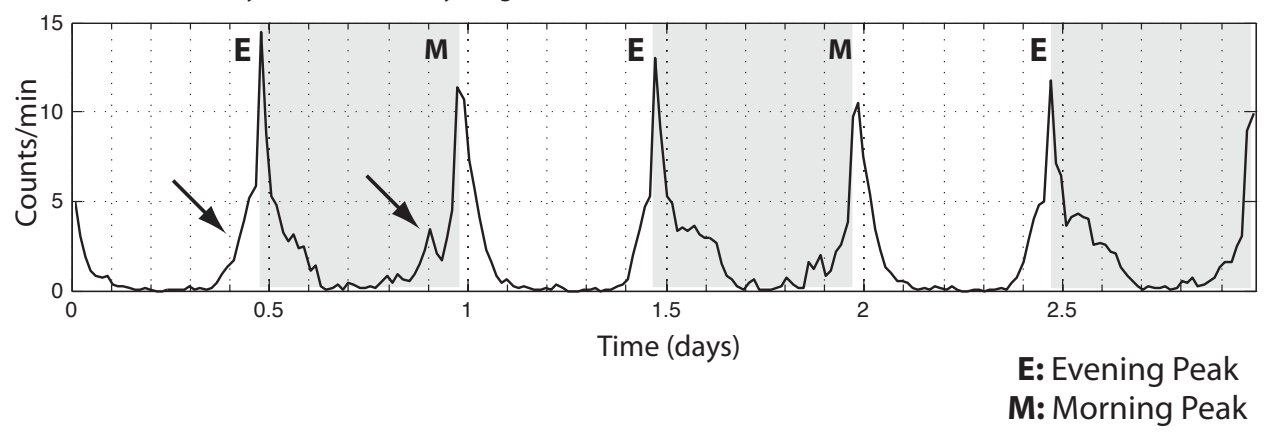

Figure 19: Circadian locomotor activity profiles of young Drosophila expressing WT- $\alpha S$ and TP- $\alpha S$

(A) Time-series circadian activity profile of the young flies (3-day old) expressing WT$\alpha \mathrm{S}$ in DA neurons. (B) Time-series circadian activity profile (3 days) of the young flies (3-day old) expressing TP- $\alpha \mathrm{S}$ in DA neurons. Activity profiles were recorded in the first week after eclosion. Experiments (Phase A according to the Figure 17) were done under the LD (12h: $12 \mathrm{~h}$ ) conditions; $\mathrm{n}=32$ in each experiment. Light and shaded regions inside the large rectangles represent alternating light/dark cycles respectively. Before every lights-on/off transition, both WT- $\alpha \mathrm{S}$ and TP- $\alpha \mathrm{S}$ flies anticipate (black arrows) the predictable morning and evening transitions (i.e. their locomotor activity starts increasing few hours before the actual lights-on/off transition and reaches peak exactly at the time of lights-on/off transition due to startle response). Anticipation is because of the learned memory of circadian clock during entrainment. Notice that there is no significant difference in the anticipatory behavior of young flies expressing the WT- $\alpha \mathrm{S}$ or TP- $\alpha \mathrm{S}$. 
In contrast to the locomotor activity profiles of young flies, the corresponding profiles of the 30-day old flies that express WT- $\alpha \mathrm{S}$ or lacZ (Figure 20A, B) were significantly different from those of flies which express TP- $\alpha \mathrm{S}$ (Figure 20C) in DA neurons as shown in the overlay profiles (Figure 20D,E). On the other hand old A53T- $\alpha$ S expressing flies have very subtle effect on circadian locomotor activity (see Figure 26 in the Appendix). However, the results demonstrate that the TP- $\alpha \mathrm{S}$ expressing old animals have lost the anticipatory behavior completely (black arrows in Figure 20).

\section{(on the next page)}

Figure 20: Circadian locomotor activity profiles of old Drosophila expressing WT$\alpha S$ and $T P-\alpha S$

(A) Time-series circadian activity profile of the 30-day old flies expressing WT- $\alpha \mathrm{S}$ in DA neurons; $n=45$. (B) Time-series circadian activity profile of the 30-day old flies expressing lacZ in DA neurons; $n=28-32$. (C) Time-series circadian activity profile of the 30-day old flies expressing TP- $\alpha \mathrm{S}$ in DA neurons; $n=44$. (D) Overlay of the activity profiles of WT- $\alpha \mathrm{S}$ (green) and TP- $\alpha \mathrm{S}$ (red). (E) Overlay of the activity profiles of WT$\alpha \mathrm{S}$ (green) and lacZ (blue). Experiments (Phase A according to the Figure 17) were done under the LD (12h: 12h) conditions. Black arrows in each rectangle point the anticipatory behavior. Thirty-day old TP- $\alpha \mathrm{S}$ expressing flies (red profile) fail to anticipate (i.e. the loss of learned circadian memory) the predictable lights-on/off transition; they only have a startle response to the sudden changes in environment during the transitions. On the other hand 30-day old WT- $\alpha \mathrm{S}$ flies (green profile) have normal anticipation like lacZ expressing (blue profile) control animals. 


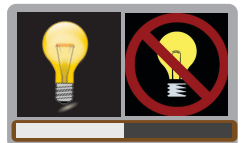

\begin{tabular}{|c|c|}
12 hours & 12 hours \\
\hline Lights ON & Lights OFF $(12 \mathrm{~h}: 12 \mathrm{~h})$
\end{tabular}

A

$\downarrow$

M: Morning Peak

Circadian Activity Profile of WT-aS (old)

E: Evening Peak
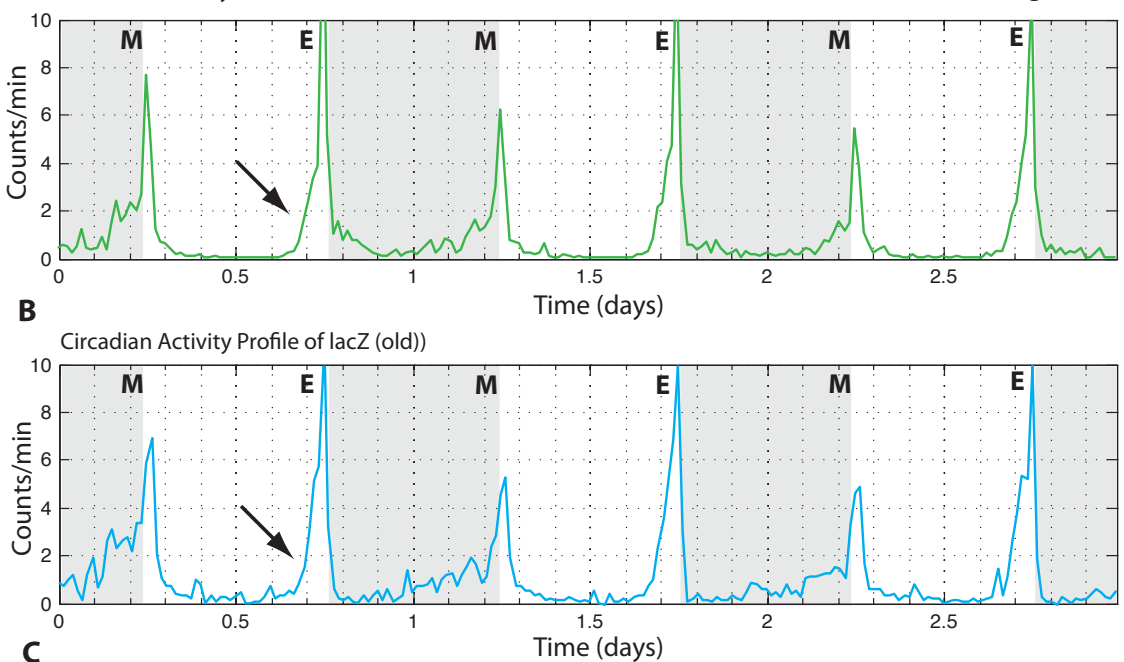

Circadian Activity Profile of TP-aS (old))

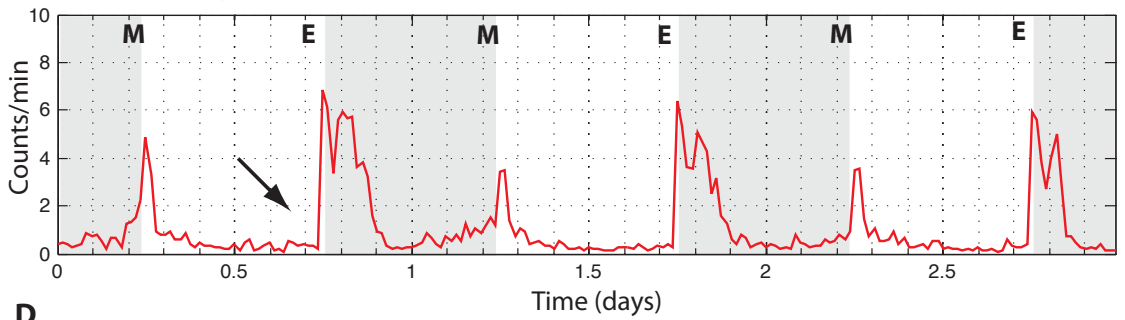

D
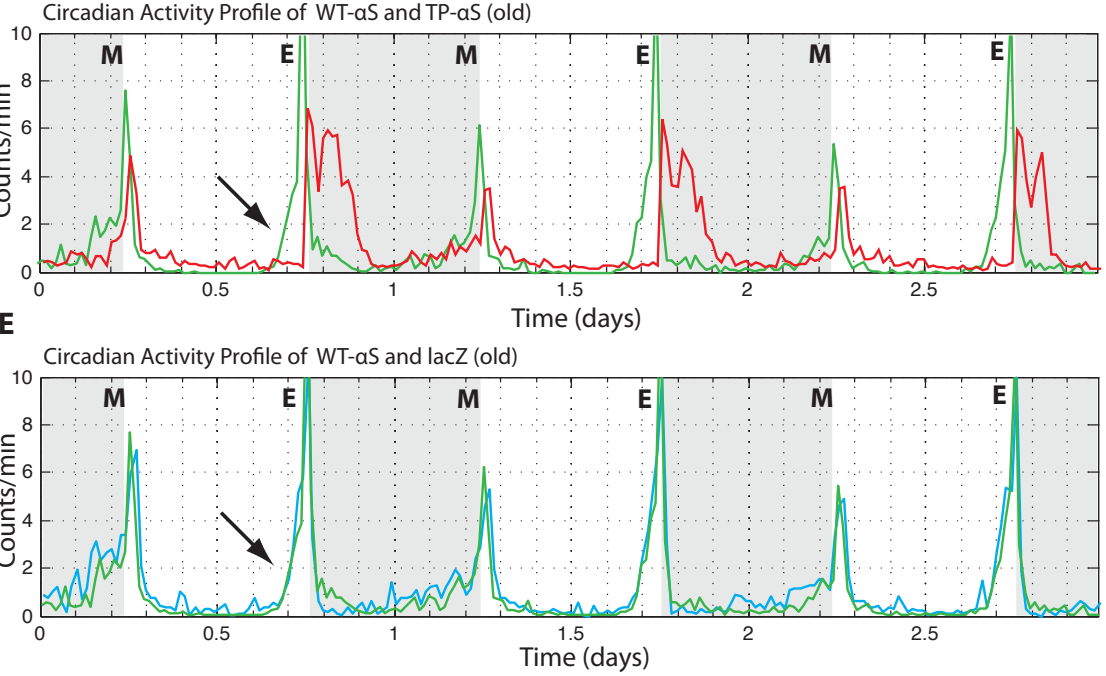
To assess the effects of WT- $\alpha \mathrm{S}$ and TP- $\alpha \mathrm{S}$ expression on central circadian clock, I also monitored circadian locomotor activities under the constant dark (DD) condition (Phase B experiment according to Figure 17) of young (3 days after hatching) and old (30-day after hatching) flies that express WT- $\alpha \mathrm{S}$ and TP$\alpha \mathrm{S}$ in neurons (schematic presentation in Figure 21A). The circadian activity of young flies expressing WT- $\alpha \mathrm{S}$ and TP- $\alpha \mathrm{S}$ is not perturbed under the continuous dark/free run condition, as shown by both the preserved pattern of activity in double-plotted actograms (Figure 21B, C) and normal periodicity (roughly 24 hours) from the chi-square periodograms (Figure 21D, E). In contrast, however, the activity pattern of aged 30-day old flies expressing oligomer-forming TP- $\alpha \mathrm{S}$ is perturbed. Double-plotted actograms (Figure 21F, G) show that the 30-day old flies expressing TP- $\alpha \mathrm{S}$ have an altered phase as compared to the WT- $\alpha \mathrm{S}$ expressing animals. Also the chi-square periodograms (Figure 14H, I) show that flies expressing TP- $\alpha \mathrm{S}$ have highly affected circadian periodicity (T) of 26.7 hours as compared to the normal 23.7 hours periodicity (T) of WT- $\alpha \mathrm{S}$ flies. In summary, flies expressing TP- $\alpha \mathrm{S}$ in DA neurons show a severe age dependent circadian clock activity defect as shown by both an altered circadian activity pattern and circadian periodicity.

(on the next page)

Figure 21: Age dependent circadian defects in Drosophila expressing $\mathrm{TP}-\alpha \mathrm{S}$ in the DA neurons

(A) Schematic of the procedure used for circadian experiment. Flies were initially entrained in LD conditions before switching to the 'free run' under DD conditions (Phase B experiment according to the Figure 17). (B-C) Actograms of the young adult flies (oneweek) expressing WT- $\alpha \mathrm{S}$ and TP- $\alpha \mathrm{S}$ under the DD conditions. (D) Chi-square periodogram of the young flies expressing WT- $\alpha \mathrm{S}$; periodicity of circadian rhythms $(\mathrm{T})=$ 23.8h. (E) Chi-square periodogram of the young flies expressing TP- $\alpha \mathrm{S}$; periodicity of circadian rhythms $(\mathrm{T})=23.8 \mathrm{~h}$. (F-G) Actograms of 30-day old flies expressing WT- $\alpha \mathrm{S}$ and TP- $\alpha$ S under the DD conditions. (H) Chi-square periodogram of the 30-day old flies expressing WT- $\alpha \mathrm{S}$; periodicity of circadian rhythms $(\mathrm{T})=23.7 \mathrm{~h}$. (I) Chi-square periodogram of the 30-day old flies expressing TP- $\alpha \mathrm{S}$; periodicity of circadian rhythms $(\mathrm{T})=26.7 \mathrm{~h} . \mathrm{n}=32-58$ flies in each experiment. In the double-plotted actograms, $\mathrm{x}$-axis 
represents time of the day (in the span $48 \mathrm{hrs}$ ) and each stair on the y-axis represents activity-histogram on the corresponding day. In the chi-square periodograms, x-axis represents the time in hours and y-axis represents the amplitude of locomotor activity wave.

A
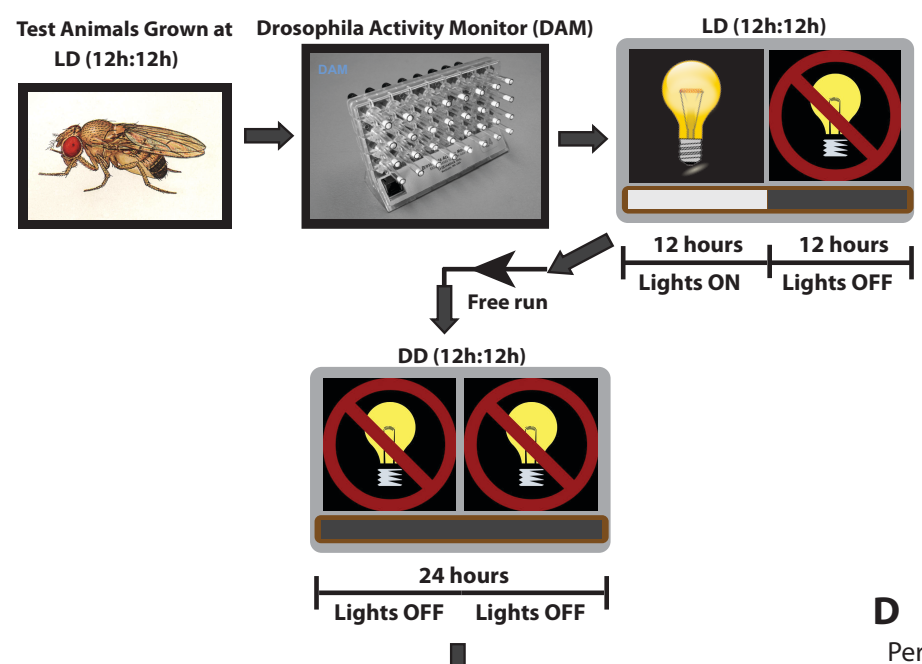

\section{D}

B

C

Actogram of WT-aS (young)

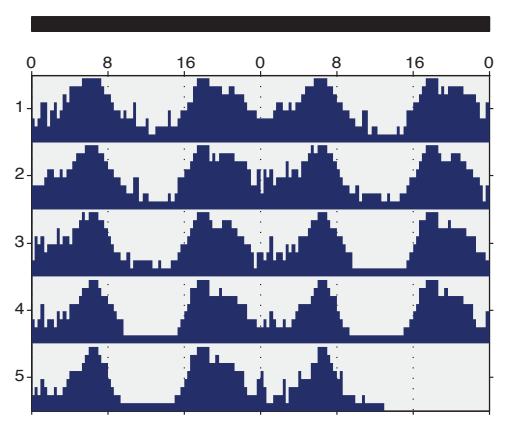

Actogram of TP-aS (young)

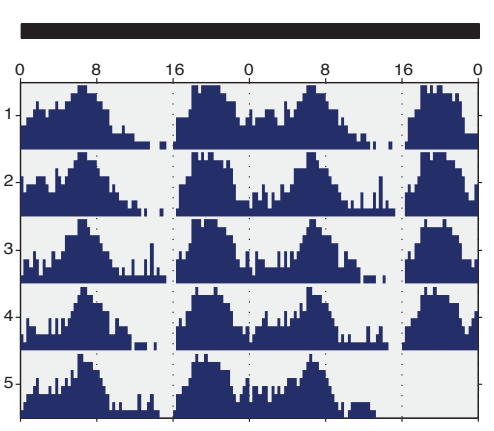

Periodograms (young)

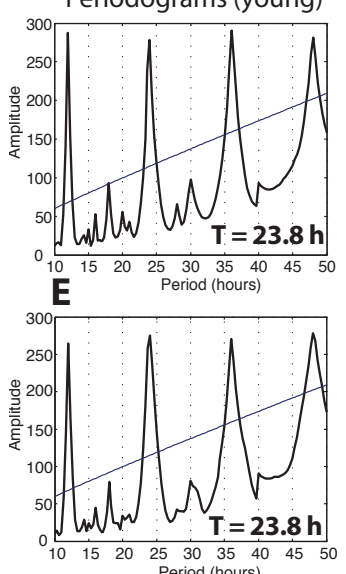

H

Periodograms (old)

$$
\mathbf{F}
$$

Actogram of WT-aS (old)

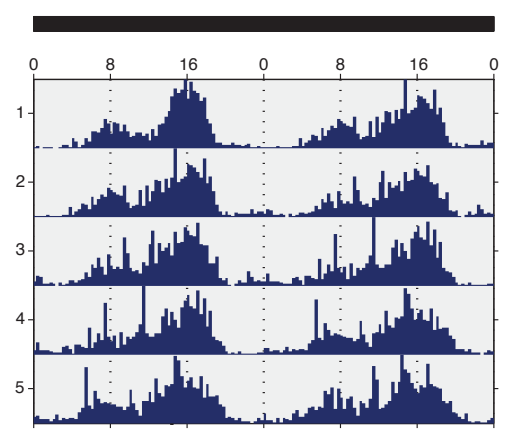

$\mathbf{G}$

Actogram of TP-aS (old)

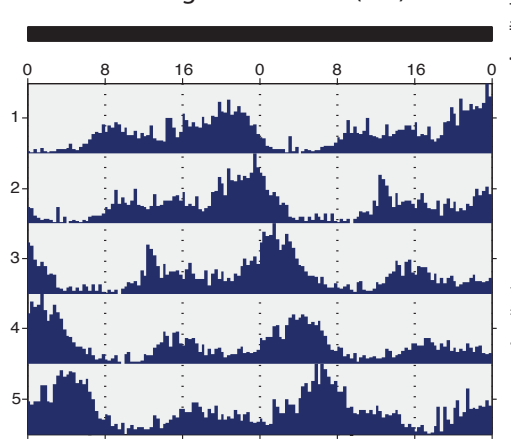




\subsection{Circadian defects in response to $\mathrm{TP}-\alpha \mathrm{S}$ expression can be mimicked by $D A$ neuron inactivation}

To understand and attribute the specificity of circadian defects caused by neurotoxic and oligomer-forming TP- $\alpha \mathrm{S}$ expression to the dopaminergic system, I have genetically reduced the activity of DA neurons (see Figure 22) by expressing $\mathrm{EKO} / \mathrm{Kir}$ 2.1. The corresponding transgene contains a mutant Shaker potassium channel that decreases synaptic excitability and output of the DA neurons (Baines et al., 2001; White et al., 2001). Conversely, I have also targeted the expression of the bacterial sodium channel $(\mathrm{NaChBac})$ in $\mathrm{DA}$ neurons, which was shown previously to interfere with DA neuronal function by increasing their excitability (Luan et al., 2006). For these experiments I used the TH-Gal4 transgene to drive the expression of the UAS-bearing $\mathrm{EKO} / \mathrm{Kir} 2.1$ and $\mathrm{NaChBac}$ transgenes specifically in the DA neurons of central nervous system (Friggi-Grelin et al., 2003).

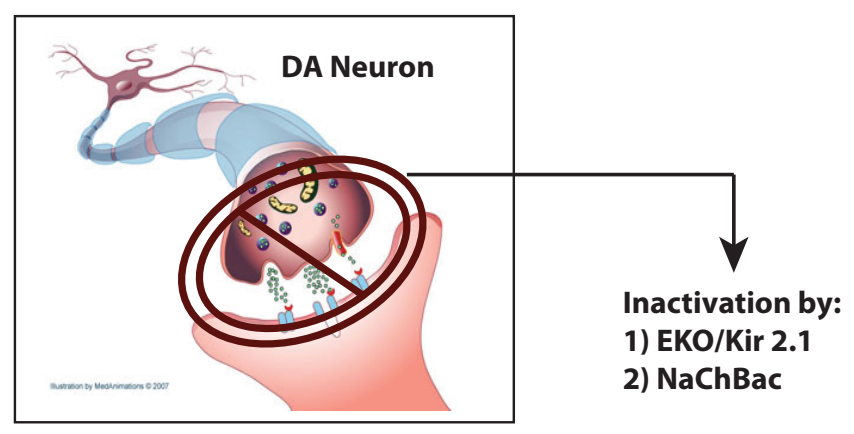

Figure 22: Schematic representation of inactivation/attenuation of the Dopaminergic (DA) neurons

Genetically targeting the expression of one copy of EKO/Kir 2.1 (mutant Shaker potassium channel) or $\mathrm{NaChBac}$ (bacterial sodium channel) specifically to the DA neurons inactivates the neurons (Luan et al., 2006; White et al., 2001), and allows us to study DA dependent behaviors in Drosophila. 
The pattern of circadian activity shown in double-plotted actograms (Figure $23 \mathrm{~B}, \mathrm{D}$ and $23 \mathrm{C}, \mathrm{E})$ indicates that the inactivation of DA neurons caused a phase shift in circadian clock activity (Figure 23D, E) similar to the phase shift caused by the expression of TP- $\alpha \mathrm{S}$ (Figure 23B, C). Also the period of circadian rhythmicity (T) as calculated by chi-square periodograms show that the normal 24-hrs period $(\mathrm{T})$ is increased in the flies with inactivated/attenuated DA neurons (Figure $23 \mathrm{G}, \mathrm{H} ; 27.6$ hours in EKO expressing flies, and 27 hours in $\mathrm{NaChBac}$ expressing flies). Thus, the periodicity in DA inactivated animals is altered as has been observed with the flies that express the TP- $\alpha \mathrm{S}$ variant in DA neurons (Figure 23F).

(on the next page)

Figure 23: Circadian defects caused by TP- $\alpha S$ mimic the effects of DA neuron inactivation by using genetic methods

(A) Schematic of the procedure used for circadian experiment. Flies were initially entrained in LD conditions before switching to the 'free run' under DD conditions (Phase B experiment according to the Figure 17). (B-C) Actograms of 30-day old flies expressing $\mathrm{TP}-\alpha \mathrm{S}$ under the DD conditions, $\mathrm{n}=58$. (D) Actogram of flies with EKO/Kir2.1 (= the mutant Shaker potassium channel) inactivated DA neurons under the DD condition, $n=32$. (E) Actogram of flies with NaChBac (bacterial sodium channel) inactivated DA neurons under the DD conditions, $n=32$. (F) Chi-square periodogram of the 30-day old flies ("old") expressing TP- $\alpha \mathrm{S}$; periodicity of circadian rhythms $(\mathrm{T})=$ 26.7h. (G) Chi-square periodogram of flies with the inactivated DA neurons by EKO/Kir2.1 expression; periodicity of circadian rhythms $(\mathrm{T})=27.6 \mathrm{~h}$. (H) Chi-square periodogram of flies with the inactivated DA neurons by $\mathrm{NaChBac}$; periodicity of circadian rhythms $(T)=27 \mathrm{~h}$. In the double-plotted actograms, $\mathrm{x}$-axis represents time of the day (in the span $48 \mathrm{hrs)}$ ) and each stair on the y-axis represents activity-histogram on the corresponding day. In the chi-square periodograms, $x$-axis represents the time in hours and y-axis represents the amplitude of locomotor activity wave. 
A Test Animals Grown at Drosophila Activity Monitor (DAM)

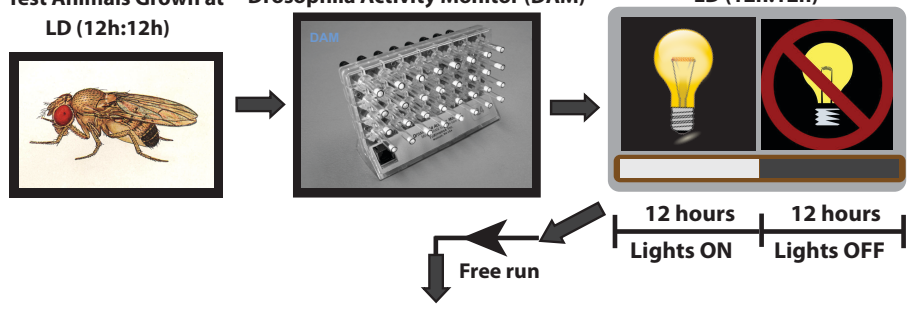

LD (12h:12h)
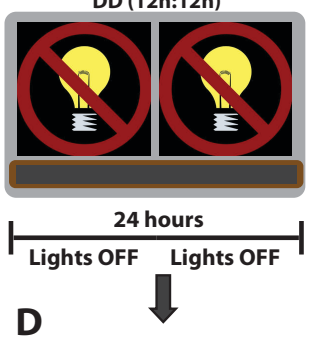

\section{B}

Actogram of TP-aS (old)

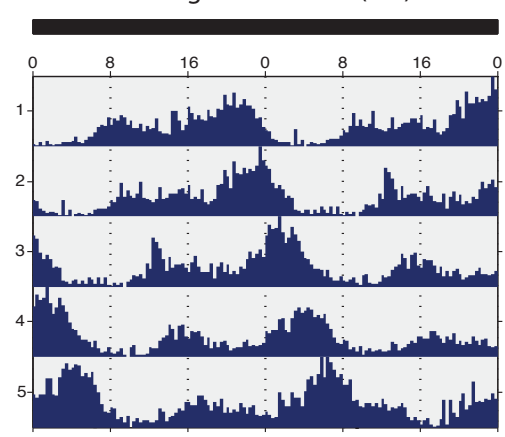

C Actogram of TP-aS (old)

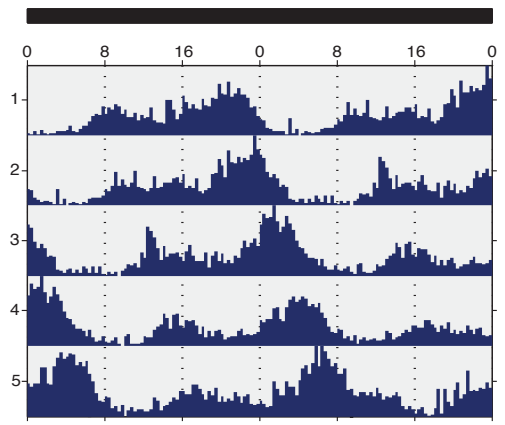

Inactivation of DA neurons with EKO

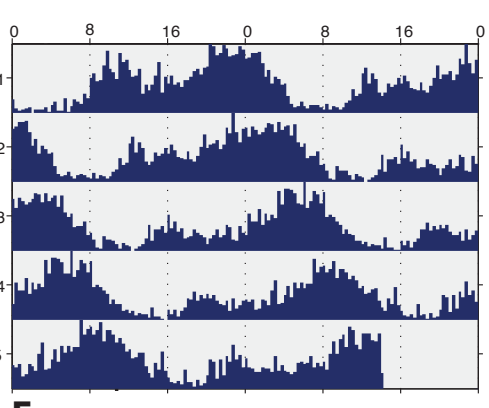
E

Inactivation of DA neurons with $\mathrm{NaChBac}$

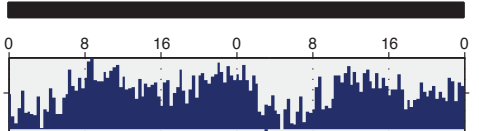

$\mathbf{F}$

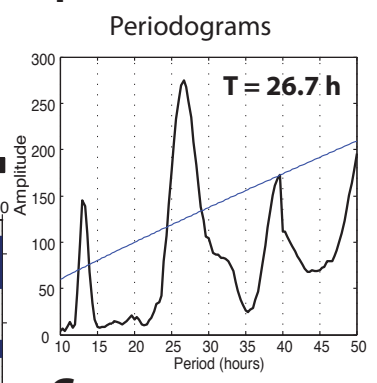

G
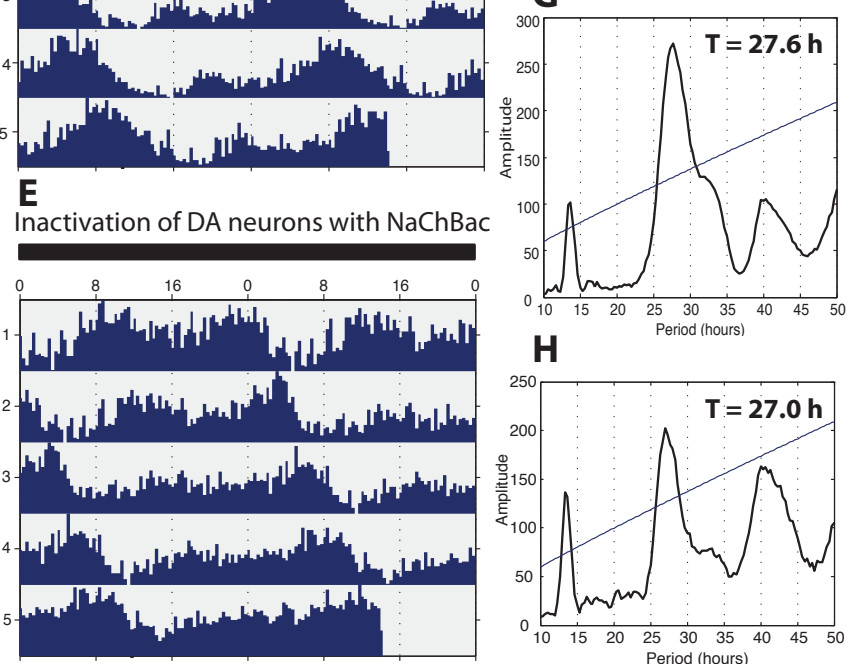


\subsection{Defective localization of neurotoxic $\alpha S$ variants to the synaptic terminals in vivo}

$\alpha \mathrm{S}$ is a presynaptic protein that localizes specifically to the axon terminals of neurons (Iwai et al., 1995; Maroteaux et al., 1988; Takamori et al., 2006). $\alpha \mathrm{S}$ is also known to have an important role in the synaptic function (Chandra et al., 2005) and hence, synucleinopathies are widely deemed as 'synaptopathies'. In addition, small diffusible pre-fibrillar oligomers formed by the aggregating proteins which are associated with neurodegeneration (for e.g. amyloid- $\beta$ oligomers) are known to adversely affect synaptic structure and function (reviewed in Haass and Selkoe, 2007).

As a first step towards understanding the possible mechanism of synaptic dysfunction caused by the oligomer-forming TP- $\alpha S$ and human PD associated A53T- $\alpha \mathrm{S}$, I started to examine the synaptic behavior of neurotoxic $\alpha \mathrm{S}$ variants along with WT- $\alpha \mathrm{S}$, using Drosophila larval neuromuscular junction (NMJ) as a model. NMJ is one of the most popular genetic model to investigate synapse physiology, functional and structural plasticity of the synapse (Catalina Ruizcanada, 2006). In order to test whether $\alpha \mathrm{S}$ variants interfere with any of these processes, I expressed the $\alpha \mathrm{S}$ variants in larval NMJs using the motor-neuron specific driver OK6-Gal4. Expression and localization of the protein was probed by fluorescent immunostaining with the anti- $\alpha \mathrm{S}$ antibody (in collaboration with $\mathrm{S}$. Sigrist's lab). As summarized in the Figure 24, TP- $\alpha \mathrm{S}$ and A53T- $\alpha \mathrm{S}$ failed to localize to the synaptic terminals, whereas WT- $\alpha$ S localized to the expected site, i.e. the synaptic terminals as shown by its co-localization with a synaptic marker called Bruchpilot (BRP). Thus, one aspect of the $\alpha \mathrm{S}$ variants is that they fail to properly localize at the synaptic terminals of the NMJs. 

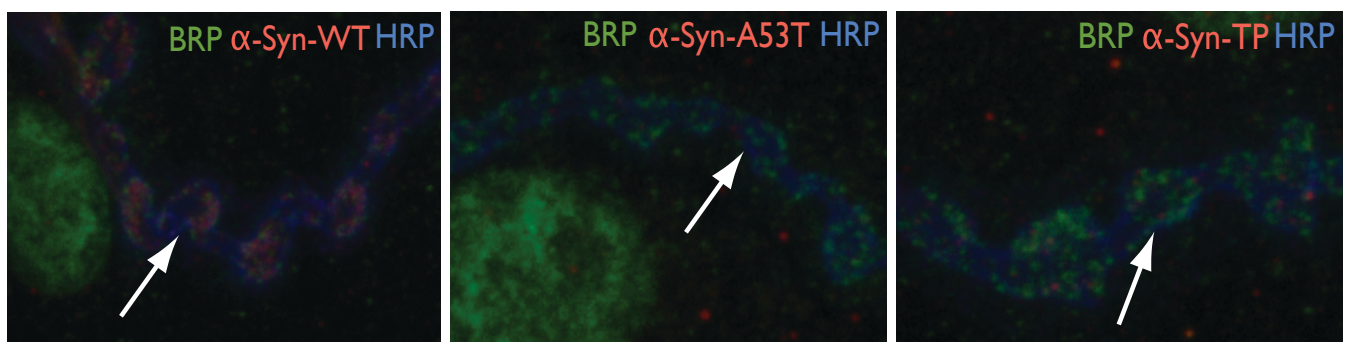

Figure 24: Defective localization of $\alpha \mathrm{S}$ variants to the synaptic terminals

Confocal images of the immunostained larval NMJs (neuromuscular junctions) expressing WT- $\alpha \mathrm{S}$ (left panel), A53T- $\alpha \mathrm{S}$ (middle panel), and TP- $\alpha \mathrm{S}$ (right panel). NMJ preparations were stained with anti- $\alpha \mathrm{S} / \alpha$ Syn (red), anti-Horse Radish Peroxidase/HRP (blue), and anti-Bruchpilot/BRP (green) antibodies. Synaptic terminals are marked by staining with BRP (green) antibody, and NMJs are marked by the HRP (blue) antibody. WT- $\alpha \mathrm{S}$ (red) is localized to the synapses (arrow) whereas TP- $\alpha \mathrm{S}$ and A53T- $\alpha \mathrm{S}$ are not targeted to the synaptic terminals. 
RESULTS 


\section{DISCUSSION}

Lewy bodies with fibrillar aggregates of $\alpha \mathrm{S}$ are one of the hallmark pathological features of Parkinson's disease and other synucleinopathies. The clinico-pathological link between $\mathrm{PD}$ and the presence of $\alpha \mathrm{S}$ aggregates has been clearly established (Shults, 2006). The aggregates of $\alpha \mathrm{S}$ are formed as a result of the dynamic pathway of aggregation involving a step-wise assembly of the soluble pre-fibrillar oligomeric species from the misfolded $\alpha \mathrm{S}$ (reviewed in Lansbury and Lashuel, 2006). Despite recent advances made in understanding PD pathogenesis, the nature of potential pathogenic species in the aggregation pathway of $\alpha \mathrm{S}$ has remained unresolved. Accumulating body of evidence from the studies on neurodegenerative diseases such as Alzheimer's and Huntington's disease suggest that small oligomeric intermediates in the aggregation pathway, rather than the aggregates themselves, are the potent pathogenic species (reviewed in Haass and Selkoe, 2007).

\subsection{Accumulation of $\alpha S$ has a toxic gain-of function}

Synucleinopathies including PD are the protein conformational neurodegenerative disorders caused by misfolding and aggregation of the $\alpha \mathrm{S}$. Misfolding and aggregation of $\alpha \mathrm{S}$ is influenced and promoted by genetic and sporadic factors. Though $\alpha \mathrm{S}$ is a presynaptic protein highly enriched in the brain, $\alpha \mathrm{S}$ knockout mice show either no or subtle (and opposite) effects on the synaptic 
transmission (Abeliovich et al., 2000; Cabin et al., 2002; Chandra et al., 2004; Yavich et al., 2004). However, overexpression of the $\alpha \mathrm{S}$ blocks endoplasmic reticulum (ER)-to-Golgi vesicular traffic (Cooper et al., 2006; Gitler et al., 2008) and reduces neurotransmitter release by inhibiting synaptic vesicle reclustering after endocytosis (Cooper et al., 2006; Nemani et al., 2010). Factors that promote accumulation of $\alpha \mathrm{S}$ by affecting its steady-state levels either by inhibiting cellular protein degradation machinery or duplication/triplication of the $\alpha \mathrm{S}$ gene locus result in the early-onset PD (Ibanez et al., 2004; Singleton et al., 2003). In addition, recent genome-wide association studies have unequivocally revealed that SNP (single nucleotide polymorphism) variations within the $\alpha \mathrm{S}$ and LRRK2 (leucine-rich repeat kinase 2) gene loci contribute to the etiology of sporadic PD (Simon-Sanchez et al., 2009). Together, these studies strongly suggest that $\alpha \mathrm{S}$ accumulation has a gain-of-toxic-function, but do not provide any specific clue regarding the cause of toxic function.

\subsection{Soluble oligomers of $\alpha$-synuclein are the likely culprits in PD pathogenesis}

It is clear that there is a well-known link between $\alpha \mathrm{S}$ accumulation and PD. But is it possible that mere accumulation of monomeric $\alpha \mathrm{S}$ causes the neurodegenerative disease? The following facts indicate (but do not prove) that monomeric form of $\alpha \mathrm{S}$ is non-toxic: (i) $\alpha \mathrm{S}$ is highly expressed in the normal brain, (ii) mutations that cause human disease don't significantly alter the native structure of the protein, but reduce its stability and hence its steady-state level in vivo while accelerating its aggregation in vitro (Conway et al., 1998; Conway et al., 2000; El-Agnaf et al., 1998; Greenbaum et al., 2005), and (iii) the cell death in neurodegenerative diseases exhibits stochastic behavior and seems to be highly dependent on the expression levels of the aggregation-prone proteins like $\alpha \mathrm{S}-$ that is, small changes in expression levels have a significant effect on the disease onset and progression (Perutz and Windle, 2001). Evidence gathered from the studies on PD and other neurodegenerative diseases also supports that monomeric forms of the $\alpha \mathrm{S}$ and other aggregation-prone proteins are most-likely non-toxic. 
However, post-translational modifications on monomeric $\alpha \mathrm{S}$ may play an important role in the modulation of neurotoxicity, as exemplified by the opposing effects of Y215 and S129 phosphorylation in the C-terminal region (Chen and Feany, 2005; Chen et al., 2009).

Having discussed the possibility of monomeric $\alpha \mathrm{S}$ being non-toxic, I will now briefly examine the pathogenicity of insoluble fibrillar aggregates of $\alpha \mathrm{S}$. Presence of insoluble fibrillar aggregates of $\alpha \mathrm{S}$ in the brains of patients is linked to the PD and other synucleinopathies. Considering the propensity of $\alpha \mathrm{S}$ to aggregate, though it was hypothesized and shown that aggregation of proteins apparently triggers a cascade of events that would finally result in the neurodegeneration and disease (reviewed in Hardy and Selkoe, 2002; Lansbury and Lashuel, 2006), recent studies suggest that fibrils may not be the potent pathogenic species (Arrasate et al., 2004; Ross and Poirier, 2004). In addition, lack of correlation between the rate of fibril and inclusion body formation with neurotoxicity (Chen and Feany, 2005; Outeiro and Lindquist, 2003; Volles and Lansbury, 2007), and the presence of amyloid plaques containing $\alpha \mathrm{S}$ in the cortex of many cognitively normal 70-year-olds indicates that apparently fibrils are not the pathogenic species in the aggregation pathway of $\alpha \mathrm{S}$ (Dickson et al., 1992). Though it is apparently clear that fibrils or inclusion bodies are not the principal pathogenic agents, one should not exclude the possibility that they may physically obstruct or interfere with normal cellular traffic to some extent. Conversely, strong support for the non-toxic nature of fibrils comes from a circumstantial evidence which indicates that inclusion bodies/fibrils can indeed act as protective repositories in the brain by sequestering pathogenic misfolded and abnormal proteins (Caughey and Lansbury, 2003; Tompkins and Hill, 1997).

Seemingly innocuous nature of $\alpha \mathrm{S}$ monomers and fibrils or inclusion bodies raises the high possibility of soluble pre-fibrillar oligomers being principal pathogenic agents in the neurodegeneration of PD and other synucleinopathies. Indeed, experimental evidence from the studies on aggregation-prone proteins like Amyloid- $\beta$, Huntingtin, and Ataxin-I, which play a key role in prominent 
neurodegenerative disorders, shows that soluble pre-fibrillar oligomeric forms are the potential pathogenic species responsible for neurotoxicity (Arrasate et al., 2004; Cummings et al., 1999; Haass and Selkoe, 2007; Schaffar et al., 2004). Taking it further, soluble amyloid oligomers of $A \beta$ are even known to interfere with the synaptic functions and alter electrical properties of the neuronal membranes (Cirrito et al., 2005; Snyder et al., 2005; Walsh et al., 2002). In addition, the early-onset Parkinson's disease associated $\alpha \mathrm{S}$ mutants, A30P, A53T, and $\mathrm{E} 46 \mathrm{~K}$, accelerate/promote oligomerization but not the rate of fibril formation by $\alpha \mathrm{S}$ (Conway et al., 2000). This common property of increased oligomerization of the different human PD-causing $\alpha \mathrm{S}$ mutants suggests that in fact pre-fibrillar oligomers of $\alpha \mathrm{S}$ in the aggregation pathway might play a vital pathogenic role in conferring toxicity to neuronal systems in the brain. It is also well-known that aggregation-prone proteins including $\alpha \mathrm{S}$ involved in various neurodegenerative diseases form annular pore-like pre-fibrillar oligomeric structures which share some morphological features both in vitro and in vivo (Lansbury and Brice, 2002; Lashuel and Lansbury, 2006; Pountney et al., 2004). These diffusible annular amyloid pore-like oligomers can impair presynaptic functions, neuronal and mitochondrial membranes; in addition, they can promote their own accumulation by inhibiting proteasome (Kitada et al., 1998; Lansbury and Brice, 2002; Leroy et al., 1998). However, the role of pre-fibrillar oligomers of $\alpha \mathrm{S}$ in PD pathogenesis is still unclear. It needs to be further studied and established systematically by linking in vitro and in vivo studies.

Precisely ascertaining the pathogenic nature of soluble-oligomers of $\alpha \mathrm{S}$ in vivo is confronted by three potential problems: (i) dynamic nature of $\alpha \mathrm{S}$ aggregation, (ii) transient and labile nature of pre-fibrillar oligomers, and (iii) lack of $\alpha \mathrm{S}$ variants with native-like structural properties that can form more pre-fibrillar oligomers with less/no fibrils. Since the metastable oligomeric species of $\alpha \mathrm{S}$ are very difficult to assess and too unstable to characterize (Kim et al., 2009) relative importance of these oligomers in PD pathogenesis is still unknown (Lansbury and Lashuel, 2006). In this conflicting background of uncertainties, my study is aimed 
at addressing the key pathogenic role of soluble-oligomers of $\alpha \mathrm{S}$. Towards this aim, I adopted a systematic approach to correlate in vitro oligomer formation with the in vivo pathogenicity of different rationally designed $\alpha \mathrm{S}$ mutations in Drosophila.

\subsection{Formation of more pre-fibrillar oligomers by the TP- $\alpha S$ and $A 56 P-\alpha S$ variants}

New $\alpha S$ variants were rationally designed based on the structural information of $\alpha \mathrm{S}$ (Section 3.1, Figure 8) in order to circumvent the problems outlined above for ascertaining the potential pathogenic nature of soluble prefibrillar oligomers of $\alpha \mathrm{S}$ (Section 4.2). The goal of rational design was to enrich the oligomers of $\alpha \mathrm{S}$ or lock the $\alpha \mathrm{S}$ in metastable oligomeric state by impinging on the cross- $\beta$ structure to destabilize the formation of $\alpha \mathrm{S}$ fibrils. Therefore, considering the flexibility of $\alpha \mathrm{S}$ backbone in monomeric state and the location of $\beta$ strands in amyloid fibrils (Bertoncini et al., 2005; Heise et al., 2005a; Vilar et al., 2008), selective replacement of the Alanine residues with Proline ( $\beta$ strand breaker) was done in the key N-terminal regions of $\alpha \mathrm{S}$. The amino acid residues A30, A56, and A76 present in the N-terminal region were chosen not only with respect to the structural reasons (see, Section 3.1; Figure 8) but also because of the lately discovered crucial functional role of $\mathrm{N}$-terminal region in mediating the synaptic toxicity caused by $\alpha \mathrm{S}$ overexpression (Nemani et al., 2010). In compliance with the rational-design principle, TP- $\alpha \mathrm{S}$ and $\mathrm{A} 56 \mathrm{P}-\alpha \mathrm{S}$ variants formed high amounts of pre-fibrillar oligomers and showed delayed fibrillation kinetics in vitro (Section 3.2; Figure 8 B, C; Karpinar et al., 2009). Strikingly, TP$\alpha \mathrm{S}$ didn't form any fibrils under the experimental conditions used for ThT aggregation assay, and A56P- $\alpha \mathrm{S}$ formed only small amounts of fibrils towards the end of assay albeit rather with delayed lag phase (Figure $8 \mathrm{~B}$ ).

Though TP- $\alpha \mathrm{S}$ and A56P- $\alpha \mathrm{S}$ variants have the properties that are quintessential to test the hypothesis of whether pre-fibrillar oligomers of $\alpha \mathrm{S}$ are potential pathogenic species in the neurodegeneration of $\mathrm{PD}$, it is also desirable 
that the chosen point mutations do not alter the native structural properties of $\alpha \mathrm{S}$. As natively unfolded WT- $\alpha \mathrm{S}$ is stabilized by the long-range interactions and pseudo-secondary structures, we studied the overall secondary and tertiary structural changes in the TP- $\alpha \mathrm{S}$ and $\mathrm{A} 56 \mathrm{P}-\alpha \mathrm{S}$ variants by using Circular Dichroism (CD) spectroscopy and liquid-state NMR spectroscopy. Fortuitously, we found no marked changes in the structures of $\alpha \mathrm{S}$ variants in comparison with the WT- $\alpha \mathrm{S}$ as reported in my published work (Karpinar et al., 2009). In addition, $\mathrm{TP}-\alpha \mathrm{S}$ and A56P- $\alpha \mathrm{S}$ both adopt helical conformation when bound to small unilamellar vesicles (SUVs), which is a well-studied property of the WT- $\alpha \mathrm{S}$ (see details in Karpinar et al., 2009). Adoption of helical conformation when bound to SUVs indicates that the mutations introduced in $\alpha \mathrm{S}$ variants do not compromise the intrinsic conformational flexibility or structural plasticity of the protein that may be required to play a functional role in binding to the neuronal or synaptic vesicle membranes. Taken together, the $\alpha \mathrm{S}$ variants TP- $\alpha \mathrm{S}$ and A56P- $\alpha \mathrm{S}$ show very close structural, and possibly functional, kinship with the native WT- $\alpha \mathrm{S}$ in their soluble monomeric form.

Given a high structural resemblance of the TP- $\alpha$ S and A56P- $\alpha$ S to the WT$\alpha S$ in their monomeric form but significant differences in soluble pre-fibrillar oligomer formation and delayed fibrillation kinetics (summarized in Figure 9), do they also share structurally similar aggregation intermediates and follow normal (on-pathway) aggregation pathway as WT- $\alpha \mathrm{S}$ ? The pre-fibrillar oligomeric intermediates formed by TP- $\alpha \mathrm{S}$ and $\mathrm{A} 56 \mathrm{P}-\alpha \mathrm{S}$ variants can seed the fibrillation of WT- $\alpha$ S. This finding indicates that they are the normal 'on-pathway' aggregation intermediates (Karpinar et al., 2009). In contrast to the on-pathway aggregation intermediates, off-pathway pre-fibrillar intermediates are not the products of normal aggregation pathway and are known to be non-toxic (Ehrnhoefer et al., 2008). Moreover, oligomeric intermediates formed by the new $\alpha \mathrm{S}$ variants were recognized by conformation-specific A11 antibody (Figure $7 \mathrm{C}$ ) - an idiosyncratic feature of several pathogenic amyloid oligomers (Kayed et al., 2003). The A11 antibody recognizes some unknown common structural conformation present in 
the amyloid oligomers formed by different amyloid forming proteins (for e.g. A $\beta$, $\alpha S$ ) involved in the neurodegenerative diseases (Kayed et al., 2003). Together, these results indicate that $\mathrm{TP}-\alpha \mathrm{S}$ and $\mathrm{A} 56 \mathrm{P}-\alpha \mathrm{S}$ follow a normal aggregation pathway and their aggregation intermediates share similar structural properties as WT- $\alpha \mathrm{S}$.

By designing $\alpha \mathrm{S}$ variants that share similar properties with the WT- $\alpha \mathrm{S}$ in their monomeric form, but have different propensities of increased oligomerization and decreased fibrillation, we created a platform to precisely test and correlate the tendency of in vitro oligomer formation to the in vivo neurotoxicity in different animal model systems such as C.elegans, Drosophila, and rat mid brain DA neurons. In this study, I specifically generated a new Drosophila PD model to test the in vivo neurotoxicity of more-oligomer-forming $\alpha \mathrm{S}$ variants in an organism that does not contain an $\alpha \mathrm{S}$ gen in its genome.

\subsection{An improved Drosophila PD model to study the pathogenicity of oligomer-forming $\alpha S$ variants}

Drosophila has no $\alpha \mathrm{S}$ or $\alpha \mathrm{S}$-like genes in its wild type genome (Auluck and Bonini, 2002; Feany and Bender, 2000). Since PD is caused by the toxic-gain-of $\alpha \mathrm{S}$ function, lack of endogenous $\alpha \mathrm{S}$ in the genome of Drosophila offers a unique advantage of testing the pathogenicity $\alpha \mathrm{S}$ variants having different in vitro properties after introducing them into its genome. Moreover, human $\alpha \mathrm{S}$ undergoes similar processing events and post-translational modifications in the Drosophila (Chen et al., 2009). Though architecture of fly brain is different from the mammalian brain, to some extent they share considerable functional similarity both in the biological processes and, in the pathways that govern these biological processes (reviewed in Colwell, 2007; Hendricks and Sehgal, 2004; Lessing and Bonini, 2009). Hence, together with the canonical advantages offered by Drosophila as an experimental system, it has become a commendable animal model to study various human neurodegenerative disorders including PD. 
I have used the Phi-C31 based site-specific recombination system (Bischof et al., 2007) that allowed precise targeting of different $\alpha \mathrm{S}$ variants, TP- $\alpha \mathrm{S}$, A56P$\alpha \mathrm{S}, \mathrm{A} 53 \mathrm{~T}-\alpha \mathrm{S}$, and wild type/WT- $\alpha \mathrm{S}$ to the identical genomic location (Figure 10; Section 3.3; Section 2.2.2). The $\alpha \mathrm{S}$ variants, TP- $\alpha \mathrm{S}$ and A56P- $\alpha \mathrm{S}$ form more oligomers in vitro, whereas A53T- $\alpha \mathrm{S}$ accelerates or promotes oligomer formation in vitro and causes also early-onset PD in humans (Conway et al., 2000). As mentioned before, the expression levels of certain disease-associated proteins and neurodegeneration are clearly related. For example, triplication or duplication of $\alpha \mathrm{S}$ gene causes PD (Ibanez et al., 2004; Singleton et al., 2003), and overexpression to a lesser extent due to a promoter polymorphism increases an individual's risk of PD (Mueller et al., 2005; Myhre et al., 2008). In light of this sensitive connection between neurodegeneration and expression levels of $\alpha \mathrm{S}$, advantages offered by the Phi-C31 based site-specific integration system, that is similar expression-dosage (see, Figure $10 \mathrm{C}$ for the similar $\alpha \mathrm{S}$ expression levels) and avoidance of possible 'position effects', make it the best Drosophila PD model ever developed for the precise comparison of pathogenicity of different $\alpha \mathrm{S}$ variants in vivo.

In contrast, all the Drosophila PD models generated heretofore (see, Feany and Bender, 2000; Liu et al., 2008) were based on introducing human $\alpha \mathrm{S}$ gene into the Drosophila genome randomly with the help of P-element mediated transgenesis (Rubin and Spradling, 1982; Spradling and Rubin, 1982). A major caveat associated with the P-element based approach is the variability of $\alpha S$ expression caused by 'position effects', i.e. the expression level of the transgene varies in dependence of its site of genomic localization. As mentioned above, having a precise control over the expression levels of different $\alpha \mathrm{S}$ variants is of paramount importance in this type of study to allow for accurate qualitative and quantitative functional comparisons. 


\subsection{Motor deficits and reduced longevity: human PD-like features in the flies expressing pre-fibrillar oligomer-forming $\alpha S$ variants}

Climbing ability and longevity are the two straightforward preliminary assays to follow neurodegeneration in the Drosophila models of neurodegenerative diseases (reviewed in Lessing and Bonini, 2009). It is also known from the previous studies that 3-4 week-old flies expressing human PDcausing $\alpha \mathrm{S}$ mutant (A30P- $\alpha \mathrm{S}$ ), and LRRK2 (leucine-rich repeat kinase)-linked PD mutant (LRRK2-G2019S) show motor deficits in the climbing assay (Feany and Bender, 2000; Liu et al., 2008). Climbing assay measures the motor performance as a read-out of the normal ability of flies to climb up (negativegeotactic behavior). In the specially designed climbing apparatus (Figure $11 \mathrm{~A}$ ), light source on top served as an attraction cue for the flies to motivate them to climb up. As dopamine (DA) is a 'pleasure molecule' involved in the reward behavior in response to positive attraction/reward cues (Ebstein et al., 2010; Johnson and Kenny, 2010), flies with reduced levels of DA might also show affected phototactic behavior. In the climbing assay that measures both motor performance (negative-geotactic) and positive-phototactic behavior of the flies, more-oligomer-forming TP- $\alpha \mathrm{S}$ and A56P- $\alpha \mathrm{S}$ expressing flies show severe motor deficits (Figure 11 B; Section 3.4). Interestingly, the TP- $\alpha S$ flies show highly impaired motor performance compared to the A56P- $\alpha \mathrm{S}$, which corresponds to their ability to form more oligomers of $\alpha \mathrm{S}$ in in-vitro. Motor deficits in A53T- $\alpha \mathrm{S}$ flies are comparable to the deficits in A56P- $\alpha \mathrm{S}$ flies (Figure $11 \mathrm{~B}$; Section 3.4).

It seems advantages offered by the accurate control of expression levels of different $\alpha \mathrm{S}$ variants, and a new set-up that allows to simultaneously assay both negative-geotactic and positive-phototactic behavior of the flies have already manifested by detecting the sensitive differences in motor deficits caused by the overexpression of WT- $\alpha \mathrm{S}$ and human PD-linked A53T- $\alpha \mathrm{S}$. In contrast to this observation, A53T- $\alpha \mathrm{S}$ didn't show enhanced toxicity in the climbing assay compared to WT- $\alpha \mathrm{S}$ in the Drosophila PD model generated by Feany and Bender 
(2000) by randomly introducing the $\alpha \mathrm{S}$ gene into the fly genome with the help of P-element mediated transgenesis.

Like in the climbing assay decribed above, the more oligomer-forming TP$\alpha \mathrm{S}$ flies also show dramatically reduced longevity in comparison with the WT- $\alpha \mathrm{S}$ and Lac-Z expressing animals (Figure 12; Section 3.5). Though WT- $\alpha$ S expression doesn't show any significant effects on the climbing ability, it results in slightly reduced longevity compared to the Lac- $Z$ expressing animals. This is consistent with the ability of WT- $\alpha \mathrm{S}$ to cause toxicity when overexpressed for a very longtime. Absence of any motor deficits in the 30-day old post-eclosion flies expressing WT- $\alpha \mathrm{S}$ animals suggests that at similar expression levels 'more oligomer-forming' $\alpha \mathrm{S}$ variants cause early-onset of motor deficits in the flies. Furthermore, highest oligomer-forming version $\mathrm{TP}-\alpha \mathrm{S}$ showed highest toxicity in both climbing and longevity assays whereas the A56P- $\alpha \mathrm{S}$ and A53T- $\alpha \mathrm{S}$ showed comparably similar levels of toxicity, giving a direct firsthand correlation between the soluble-oligomer formation and toxicity in the preliminary assays of neurodegeneration.

\subsection{Degeneration of DA neurons in the flies expressing pre- fibrillar oligomer-forming $\alpha S$ variants}

Since age dependent loss of dopaminergic neurons (DA neurons) is the hallmark feature of PD, I followed degeneration of DA neurons in the adult Drosophila brain. The DA neuronal numbers in the DL (dorso-lateral) and DM (dorso-medial) clusters of the young flies (2 days after hatching) expressing different variants of $\alpha \mathrm{S}$ are normal and show no sign for degeneration (Figure 13 B, C; Section 3.6.1), whereas the old flies (30 days after hatching) show significant differences in the numbers of neurons (Figure $14 \mathrm{~B}, \mathrm{C}$; Section 3.6.2). I observed that flies expressing the oligomer-forming $\alpha \mathrm{S}-\mathrm{TP}$ have lost highest number of DA neurons in both DM and DL clusters. As noted in previous assays (Section 3.4, 3.5), the neurotoxicity exhibited in response to the expression of the A56P- $\alpha$ S and A53T- $\alpha$ S mutants was similar but clearly less pronounced than in response to TP- $\alpha \mathrm{S}$ expression (Figure14 B, C). In all cases, the toxic effect was 
more pronounced than that observed with WT- $\alpha \mathrm{S}$ expression, which does not cause a significant neuronal loss when compared to control animals expressing the Lac-Z marker under otherwise identical conditions (Figure 14 B, C).

Although progressive neuronal loss is an important feature of PD and other neurodegenerative diseases, some neurological impairment may reflect dysfunction rather than loss of neurons. Moreover, neuronal systems have absolutely high levels of plasticity and complex compensatory mechanisms that come into play before they finally resort to degeneration. While observed neuronal loss definitely gives a measure of the neurotoxicity exhibited by different $\alpha \mathrm{S}$ variants, it does not give any clue regarding the neuronal dysfunction caused by $\alpha \mathrm{S}$ variants. For example, though neuronal loss is comparably similar in the flies expressing A56P- $\alpha \mathrm{S}$ and A53T- $\alpha \mathrm{S}$, it is still possible that A56P- $\alpha \mathrm{S}$ causes more neuronal dysfunction than $\mathrm{A} 53 \mathrm{~T}-\alpha \mathrm{S}$ as reflected in the stronger motor deficits that were observed in the climbing assay applied. Interestingly, as expected from the effects on climbing and longevity, TP- $\alpha \mathrm{S}$ expressing flies have lost highest number of DA neurons. This finding provides support for the argument that soluble pre-fibrillar oligomers are the cause, and possibly the principal pathogenic agents causing neurodegeneration associated with PD. However, though DA neurons are most vulnerable to the $\alpha \mathrm{S}$ toxicity, neuronal degeneration in PD patients is not limited to the DA neurons but rather extends to other neural regions as the disease progresses (Farrer, 2006).

\subsection{Non-motor deficits in PD flies expressing pre-fibrillar oligomer forming $\alpha S$ variants}

The clinical presentation of PD with motor symptoms has partly overshadowed the focus on the study of non-motor symptoms of PD. However, non-motor symptoms such as sleep abnormalities and compromised circadian function (Factor, 2002; Whitehead et al., 2008) highly affect the quality of life in human PD patients as much as clinical motor symptoms. Research on the pathophysiology of non-motor symptoms has been hindered, in part, by the lack of an appropriate animal model that can reproduce the non-motor symptoms of 
PD (Sheila M. Fleming, 2009). Therefore, I've studied sleep abnormalities and circadian locomotor activity rhythms in the Drosophila PD model, both to elucidate the pathogenicity of pre-fibrillar oligomer forming variants, and to eventually develop a simple animal model for studying the pathophysiology of non-motor symptoms in Parkinson's disease.

\subsection{Sleep abnormalities in the PD flies}

Cardinal features of mammalian sleep are conserved in Drosophila (reviewed in Colwell, 2007; Hendricks and Sehgal, 2004). In the past few years, Drosophila has emerged as a commendable non-mammalian model to understand the neurogenetics of sleep (Harbison et al., 2009; Ho and Sehgal, 2005) after it was demonstrated that the quiescence in Drosophila represents a sleep-like state in flies. In addition, flies show canonical features of the vertebrate sleep: (i) increased arousal threshold or reduced responsiveness to mechanical stimuli, (ii) homeostatic drive to recover from the lost sleep, (iii) fragmented sleep in older flies, (iv) response to the drugs like caffeine and methamphetamine, (v) conserved sleep/wake signaling (cAMP and EGFR), and (vi) conserved molecular response to sleep deprivation such as up-regulation of BiP/GRP78 gene expression (Zimmerman et al., 2008).

Since sleep abnormalities are one of the prominently reported non-motor symptoms in PD patients, I have examined the sleeping behavior of flies expressing different $\alpha \mathrm{S}$ variants by analyzing various sleep parameters such as bout numbers, bout lengths, total sleep, sleep profiles, and activity index as outlined in Section 3.8 (see also Figure 15). The flies expressing 'more-oligomerforming' variants of $\alpha \mathrm{S}, \mathrm{TP}-\alpha \mathrm{S}$ and A56P- $\alpha \mathrm{S}$ show sleep abnormalities. They have a decreased number of sleep-bouts and an increased length of sleep-bouts. However, the total length of the sleep period is not altered. These findings suggest that sleep abnormalities are most likely due to the effects on the mechanisms regulating sleep initiation and maintenance (Section 3.8; Figure 16 A-C). Moreover, the effects on Drosophila sleep not only consolidate the pattern of toxicity observed in previous assays (i.e. the climbing and longevity assays as 
well as DA neuronal death), but also show that oligomer-forming $\alpha \mathrm{S}$ mutants are more efficient to cause the PD-associated effects than the wild type $\alpha \mathrm{S}$ protein. Thus, sleep abnormalities in flies expressing $\alpha \mathrm{S}$ variants can serve as a sensitive readout to correlate the degree of in vitro pre-fibrillar oligomer formation with PD-related effects in vivo (Figure 16). Among the different $\alpha \mathrm{S}$ variants tested, the TP- $\alpha \mathrm{S}$ variant stands out by causing highest intensity of sleep abnormalities (Figure $16 \mathrm{D}$ ). The effects are less pronounced in response to the expression of the A56P- $\alpha$ S variant, a finding that parallels the pre-fibrillar oligomer-formation tendencies of the two $\alpha \mathrm{S}$ variants in vitro.

Can sheer DA neuronal dysfunction that is not associated with degeneration cause sleep abnormalities? Since sleep was studied in the flies during their firstweek after eclosion and as there is no DA neurodegeneration observed in the firstweek (Section 3.6.1; Figure 13), sleep abnormalities observed in this assay is likely to be caused by DA neuronal dysfunction rather than by their degeneration. Though DA plays a similar role in sleep/wake control in both mammals and Drosophila (reviewed in Zimmerman et al., 2008), effect of the DA neuronal dysfunction on Drosophila sleep can be mechanistically explained by the fact that DA neurons send their projections to the lobes of mushroom-body (MB) (Zhang et al., 2007), a brain site shown to play a dynamic role in sleep regulation (Joiner et al., 2006; Pitman et al., 2006). In addition, a very recent study focused on elucidating a link between memory deficits and abnormal sleep in PD flies also emphasizes the pathological relevance of sleep in neurodegeneration of PD (Seugnet et al., 2009). The results shown here do not allow to distinguish between DA dysfunction and degeneration as the cause of the sleep abnormalities flies, but they suggest that the neuronal degeneration is less likely to be the cause of the sleep effects observed. In fact, my results show that flies expressing toxic variants of $\alpha \mathrm{S}$ show sleep abnormalities in a similar way like human PD patients, and the sleep deficits in Drosophila can be used a sensitive readout to correlate the prefibrillar oligomer-forming tendency of $\alpha \mathrm{S}$ in vitro to the neurotoxicity in vivo. It has been shown that pharmacological imposition of sleep can improve cognitive 
functions in a mouse model of Huntington's disease (Whalley, 2007), in light of this protective role of sleep in neurodegenerative diseases, the newly introduced Drosophila PD model provides now a valuable organism tool for possible screens in order to identify therapeutic chemical lead compounds to eventually treat the sleep abnormalities in PD patients.

\subsection{Circadian dysfunction in the flies expressing most toxic oligomer-forming TP- $\alpha \mathrm{S}$}

Disturbances in the circadian activity pattern are common among patients with severe PD and related neurodegenerative disorders (Factor, 2002; Whitehead et al., 2008). Based on the conserved features of the Drosophila circadian clock and DA neuronal signaling, it was interesting to ask whether flies with severe PDlike phenotypes also show circadian activity deficits. Moreover, dopaminergic neuronal signaling in Drosophila is predicted to have a role in translating the circadian molecular oscillations in clock cells into the locomotor activity rhythms (Hirsh et al., 2010; Suh and Jackson, 2007), and like in mammals it is implicated to have a role in the regulation of arousal or sleep/wake cycle (Dzirasa et al., 2006; Ebstein et al., 2010).

Since TP- $\alpha \mathrm{S}$ showed strongest neurotoxic effects among the $\alpha \mathrm{S}$ variants in all the behavioral assays tests applied, and led to a severe degeneration of DA neurons in the DM and DL clusters in adult fly brain, I have also examined the effects on circadian locomotor activity rhythms in the flies expressing TP- $\alpha \mathrm{S}$. As in the human PD, flies expressing TP- $\alpha \mathrm{S}$ also caused circadian deficits in an agedependent manner. The circadian locomotor activity profiles of young flies (3-day old) was not significantly affected in response to TP- $\alpha \mathrm{S}$ as compared to $\alpha \mathrm{S}$ expression, whereas the circadian locomotor activity profiles of old (30-day old) WT- $\alpha$ S and TP- $\alpha$ S flies (compare Figure 19 A,B and 20 B, C) differ significantly, i.e. the activity profile of the 30-day old TP- $\alpha \mathrm{S}$ expressing flies was aberrant when compared to the activity profiles of the WT- $\alpha \mathrm{S}$ and Lac-Z flies that served as controls. Most notably, the old TP- $\alpha \mathrm{S}$ expressing flies have lost their 
'anticipatory behavior' in response to circadian changes in the external environment (Section 3.9; Figure 20).

Furthermore, young flies expressing WT- $\alpha \mathrm{S}$ and TP- $\alpha \mathrm{S}$ have normal circadian activity pattern (Section 3.9; Figure $21 \mathrm{~B}, \mathrm{C}$ ) and circadian periodicity $(\mathrm{T}=23.8 \mathrm{~h})$ (Figure $21 \mathrm{D}, \mathrm{E})$ under the constant dark conditions (DD/free-run; see Figure 17 for the general scheme), In contrast, the old TP- $\alpha S$ expressing flies show an abnormal phase-delayed activity pattern (Figure $21 \mathrm{G}$ ) associated with an increased circadian periodicity $(\mathrm{T}=26.7 \mathrm{~h}$ ) (Figure $21 \mathrm{I}$ ) when compared to the $\alpha \mathrm{S}-\mathrm{WT}$ expressing animals (Figure $21 \mathrm{~F}, \mathrm{H}$ ).

In summary, these results show that the observed dysfunction and degeneration of DA neurons in response to the TP- $\alpha \mathrm{S}$ expression result in an altered circadian locomotor activity. This observation further strengthens the proposed role of DA neurons in the regulation of circadian locomotor activity (Hirsh et al., 2010; Suh and Jackson, 2007). However, the effects observed on circadian locomotor activity rhythms might be the result of indirect perturbations caused by the neuronal dysfunction or degeneration of the DAs, rather than a direct consequence of the dysfunctional or degenerated DA neurons. Despite prevailing mechanisms of 'degeneracy' and 'compensatory effects' for a malfunction in the brain, impairment of a single neuronal circuit can influence the functions of an entire network of inter-connected neuronal circuits. Therefore, dysfunction and degeneration of one element in response to TP- $\alpha \mathrm{S}$ expression, such as the DA neurons as shown here, can also affect a behavior that is under the control of a neural circuit and thus, we cannot decide whether TP- $\alpha$ S expression lead to circadian deficits even if the DA neurons have a role down-stream of the clock neurons as predicted by Suh and Jackson, 2007, or whether they have a direct function in the process. However, the close vicinity of the DL clusters and the so-called PDF-neurons, a group of clock neurons that express a neuropeptide called Pigment Dispersion Factor, and the spatial overlap of the PDF neuronal arborizations and DA neuronal cell bodies in the fly brain (own observation) imply that the effects on circadian locomotor activity rhythms caused by TP- $\alpha \mathrm{S}$ 
expression are more likely indirect effects. Such effects could result from an improper translation of circadian molecular oscillations in PDF neurons into the activity rhythms.

I have inactivated the DA neurons directly by using genetic methods (Section 3.10; Figure 22), and I have examined the circadian locomotor activity rhythms in the flies where DA neurons were inactivated by the targeted expression of the shaker mutant EKO/Kir 2.1 or the bacterial sodium channel NaChBac (Luan et al., 2006; White et al., 2001) in DA neurons using UAS/GAL4 system (Brand and Perrimon, 1993). The abnormal circadian activity patterns of flies expressing $\mathrm{EKO} / \mathrm{Kir} 2.1$ and $\mathrm{NaChBac}$ in the DA neurons mimic the abnormal pattern of circadian activity after TP- $\alpha$ S-expressing in old flies (Section 3.10; Figure 23 B-E) both with respect to the observed phase-delay and the abnormal circadian periodicity of about $27 \mathrm{hrs}$ instead of $24 \mathrm{hrs}$ (Section 3.10; Figure 23 F-H). These results show that the abnormal effects on circadian locomotor activities observed after the expression of pre-fibrillar oligomer forming TP- $\alpha \mathrm{S}$ are indeed specific effects due to the loss of DA neuronal functions. The results shown here, therefore, provide strong evidence for the possible role of DA neurons in translating circadian molecular oscillations into the locomotor activity rhythms. In addition, these results also indicate that we can use Drosophila as an experimental system to address the mechanism of the circadian activity deficits associated with the PD.

\subsection{Synucleinopathies are synaptopathies: defective localization of the neurotoxic $\alpha S$ variants at the synaptic terminals of larval NMJs}

Because of the presynaptic localization (George et al., 1995; Iwai et al., 1995) and predicted role of $\alpha \mathrm{S}$ in the synaptic function (Cabin et al., 2002; Chandra et al., 2004), synucleinopathies are widely deemed as 'synaptopathies'. As an initial step towards understanding of the mechanism of pathogenesis at synapse level, I have examined the localization of the different $\alpha \mathrm{S}$ variants at the synaptic terminals of larval Neuromuscular Junctions (NMJs) after their targeted 
expression in motor-neurons (Section 3.11; Figure 24). As shown in the Figure 24, the pathogenic $\alpha \mathrm{S}$ variants TP- $\alpha \mathrm{S}$ and A53T- $\alpha \mathrm{S}$ fail to properly localize at the synaptic terminals of NMJs, whereas the WT- $\alpha \mathrm{S}$ shows a normal localization pattern as observed in mammals. Although it is clearly premature to extrapolate and envisage a mechanism of neurotoxicity merely from the improper localization of pathogenic $\alpha \mathrm{S}$ variants at the motor-neuron NMJs, it is likely that the lack of proper folding and formation of pre-fibrillar oligomers of the mutant $\alpha \mathrm{S}$ variants can elicit an 'unfolded protein response' (UPR) and block the ER-to-Golgi traffic (Cooper et al., 2006), consequently affecting the endocytosis and other synaptic functions in neurons. In addition, the neuronal loss observed in the flies expressing the pathogenic oligomer-forming $\alpha \mathrm{S}$ variants can be attributed to the fact that an UPR leads to the programmed cell death in nervous system (Bredesen et al., 2006). In both cases, NMJ function would be severely impaired. This conclusion is consistent with the results of recent electrophysiological studies on hippocampal slices and rat midbrain dopamine neurons which overexpress $\alpha \mathrm{S}$, showing reduced neurotransmitter release that resulted from the inhibition of synaptic vesicle reclustering at the nerve synapse (Nemani et al., 2010) without having any obvious effects on ER-to-Golgi traffic. In fly larvae, however, the mislocalized pathogenic $\alpha \mathrm{S}$ variants had no effects on excitatory junctional currents (EJCs), paired-pulse ratios and miniature EJCs (unpublished data obtained in collaboration with S. Sigrist's Lab, FU Berlin). These results suggest that expression of $\alpha \mathrm{S}$, which is normally not present in flies, and the different prefibrillar oligomer-forming $\alpha \mathrm{S}$ variants in motor-neurons do not interfere with the process of synaptic transmission at the NMJ as observed in mammals. It is therefore unlikely, that the overexpression of $\alpha \mathrm{S}$ and $\alpha \mathrm{S}$ variants interferes with cell trafficking as proposed above or with synaptic transmission per se but rather than with a cellular function not directly involved in synaptic transmission at the NMJs. Alternatively or in addition, $\alpha \mathrm{S}$ overexpression may not interfere with the synaptic transmission system at larval Drosophila NMJ which involves the glutamatergic system only. Moreover, it is possible that pathogenic synaptic 
deficits in response to $\alpha \mathrm{S}$ and $\alpha \mathrm{S}$ variant expression can only be observed in adult Drosophila NMJs, since aging is considered as one of the risk factor associated with the PD pathogenesis. Confirmation of this proposal has to await further studies on the adult NMJs of the fly.

\subsection{Conclusion}

Studies on PD are limited to material of human patients. This limitation requires model organisms in which conclusions derived from patient studies can be critically tested and the proposed factors and the mechanisms leading to the disease can be unraveled. My results show that many aspects of PD, including sleep behavior and altered circadian rhythms can be observed in $\alpha \mathrm{S}$-expressing Drosophila, an organism that normally lacks $\alpha \mathrm{S}$ or $\alpha \mathrm{S}$-like genes in its genome. Rationally designed $\alpha S$ mutants, which form more $\alpha \mathrm{S}$ pre-fibrillar oligomers in vitro (Karpinar et al., 2009) show an inextricable direct correlation between the propensity to form more $\alpha \mathrm{S}$ pre-fibrillar oligomers in vitro and corresponding neurotoxicity in vivo (see caricatured summary in Figure 25). The results obtained with Drosophila were corroborated by results concurrently obtained with established PD models such as C.elegans (collaboration with S. Eimer's lab) and rat midbrain dopamine neurons (collaboration with S. Kuegler's lab) conclusively demonstrating that pre-fibrillar oligomers are the most toxic pathogenic species in the neurodegeneration of PD (see results in the Karpinar et al., 2009). In addition, these findings demonstrate that these model organisms can be used to assess the toxicity and biological significance of any kind of $\alpha S$ mutants, even though it is technically impossible to directly visualize and quantify the pre-fibrillar oligomers in vivo (Kim et al., 2009). 


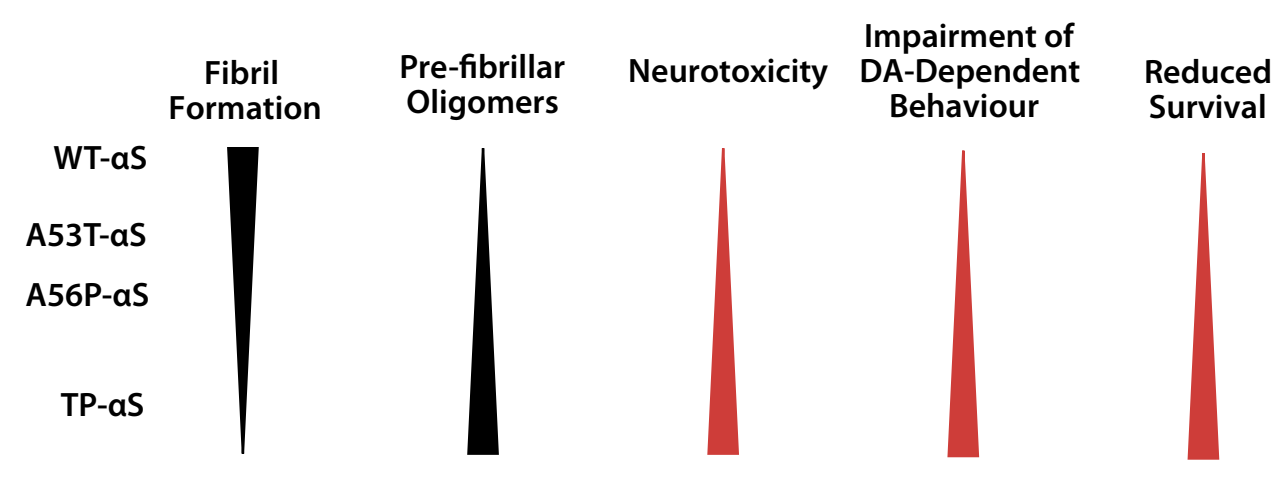

Figure 25: Caricatured representation of the conclusive summary

Representation of experimental summary of the correlation between in vitro behavior of the $\alpha \mathrm{S}$ variants (black cones) and pathogenicity in vivo (red cones) in different model systems including the Drosophila. Blunt-end (maximum) to tapered-end (minimum) of the cone represents an arbitrary quantitative range.

Drosophila has the major advantage that transgenes containing $\alpha \mathrm{S}$ or any kind of $\alpha \mathrm{S}$ mutations can be placed exactly into the same chromosomal location and thus, their expression is not affected by "position effects" that may lead to different expression levels of the transgenes. In addition to this technical improvement, my results also show that the Drosophila PD model not only be used to understand the pathophysiology of both motor symptoms and cellular aspects of the degeneration but also to examine the non-motor PD symptoms such as sleep behavior and the effects on the circadian rhythm of PD patients, possibly also including other synucleinopathies. Most importantly, this study on elucidating the role of soluble pre-fibrillar oligomers of $\alpha \mathrm{S}$ in $\mathrm{PD}$ sheds a new light on the nature of pathogenic species by showing that the $\alpha \mathrm{S}$ variants which form more prefibrillar oligomers exhibit highest neurotoxicity. It is therefore possible, to assess the biogenesis of aggregopathies and to unveil the pathology of the process mechanistically. Furthermore, organism-based assays for drug screening using the Drosophila systems come within reach. 


\section{SUMMARY AND OUTLOOK}

$\alpha \mathrm{S}$ is an abundantly expressed, natively unfolded pre-synaptic protein in the central nervous system of human brain. Although details about its function are still unknown, its aggregation in brain cells leads to neurodegeneration in the Parkinson's disease (PD) as well as other diseases collectively referred to as "synucleinopathies". Synucleinopathies are clinicopathologically characterized by the loss of DA neurons and the presence Lewy bodies enriched with $\alpha \mathrm{S}$ aggregates. It is well established that the aggregation process of $\alpha \mathrm{S}$ is the harbinger of pathogenic events that finally leads to neurodegeneration in PD and synucleinopathies. However, the nature and identity of pathogenic species in the aggregation pathway of $\alpha \mathrm{S}$ is still a matter of controversial debates. It is for example undecided whether $\alpha \mathrm{S}$ fibrils, which are the end products of the $\alpha \mathrm{S}$ aggregation, or the pre-fibrillar oligomers, representing the intermediates in the $\alpha S$ aggregation, are the effective pathogenic species. Accumulating evidence suggests that pre-fibrillar oligomers of the aggregation-prone proteins such as Amyloid- $\beta$ and Huntingtin are the potential pathogenic species, which cause the neurodegeneration of the respective diseases. Since it is believed that neurodegenerative diseases caused by or resulting in protein aggregation might have common pathogenic mechanisms, I have investigated whether the emerging paradigm of potential pathogenesis vested in pre-fibrillar oligomers holds also 
true for the $\alpha \mathrm{S}$ soluble oligomers in the pathogenesis of PD. The systematic reverse-genetics approach taken to critically address this problem involved the following steps:

- Generating $\alpha \mathrm{S}$ variants that can form more pre-fibrillar oligomers by using a structure-based rational design.

- Testing the in vitro pre-fibrillar oligomer formation of different $\alpha \mathrm{S}$ variants, and attempting to characterize them.

- Generating different animal models to test the pathogenicity of $\alpha \mathrm{S}$ variants.

- Translating the experimental observations made on the in vitro behavior of $\alpha \mathrm{S}$ variants into animal models (C.elegans, Drosophila and rat mid brain DA neurons) by asking whether the tendency of formation of pre-fibrillar oligomers in vitro can be correlated with neurotoxicity in vivo.

In this context, I have generated an improved experimental system using Drosophila melanogaster as a model organism in which questions concerning the toxicity and biological significance of $\alpha \mathrm{S}$ and pre-fibrillar $\alpha \mathrm{S}$ mutants can be addressed. $\alpha S$ variants were generated, by a structure-based rational design, which remain longer in the pre-fibrillar oligomer-forming stage than wild type $\alpha \mathrm{S}$. They include TP- $\alpha \mathrm{S}$ and $\mathrm{A} 56 \mathrm{P}-\alpha \mathrm{S}$ which showed a very close structural resemblance and similar conformational flexibility in their native monomeric form to the WT$\alpha \mathrm{S}$ but exhibited remarkable differences in their fibrillation propensities. Both TP$\alpha \mathrm{S}$ and A56P- $\alpha \mathrm{S}$ had an increased propensity to form more pre-fibrillar oligomers and decreased propensity to form fibrils in vitro. Among the different $\alpha \mathrm{S}$ variants, TP- $\alpha \mathrm{S}$ formed highest amount of pre-fibrillar oligomers followed by the A56P$\alpha \mathrm{S}$. We critically tested the pathogenicity of these 'more-oligomer-forming' variants of $\alpha \mathrm{S}$ in comparison with the effects of the WT- $\alpha \mathrm{S}$ and the A53T- $\alpha \mathrm{S}$ mutation that is known to cause human PD using in the above-mentioned different PD model organisms. 
I have generated a new and improved Drosophila PD model by introducing $\alpha \mathrm{S}$ variants with different oligomer-forming tendencies into the fly genome, which normally lacks $\alpha \mathrm{S}$ and $\alpha \mathrm{S}$-like genes. For generating the transgene-bearing flies, I have used the Phi-C31 based site-specific recombination technique to circumvent possible problems associated with different expression levels of the inserted transgenes due to so-called 'position effects' that derive from different genome integration sites. This system, therefore, allows an unbiased functional comparison of the pathogenicity of different $\alpha \mathrm{S}$ variants when expressed in the same cells of the fly.

After generating the Drosophila PD model by introducing different $\alpha \mathrm{S}$ variants into the fly genome, I have examined both motor symptoms and nonmotor symptoms that are known from PD patients. This pioneering approach allowed me to accurately compare and correlate the propensity of pre-fibrillar oligomer formation by the $\alpha \mathrm{S}$ variants to their in vivo pathogenicity. The results show that this comprehensive yet easily manageable experimental system could be useful for both to assess the mechanisms of PD pathogenesis and to screen for chemicals serving as lead compounds to develop therapeutic tools in order to intervene with PD symptoms.

In assays designed to assess neurodegeneration, climbing (= negative geotaxis and positive phototaxis) and longevity, the 'more-oligomer-forming' $\alpha S$ variants showed more severe effects than $\alpha \mathrm{S}$ expression in the corresponding cells. Consistent with its ability to form the highest amount of pre-fibrillar oligomers among the $\alpha \mathrm{S}$ variants tested, TP- $\alpha \mathrm{S}$ also showed most severe effects in both the climbing and longevity assay. With respect to its strongest effects in vivo, TP- $\alpha \mathrm{S}$ was followed by the A56P- $\alpha \mathrm{S}$ mutation and the A53T- $\alpha \mathrm{S}$ variant known to cause PD in human. In addition, flies expressing the more-oligomerforming $\alpha \mathrm{S}$ variants had lost a higher number of DA neurons in the DL and DM clusters of the adult brain, an effect that was found to be age-dependent. In contrast, WT- $\alpha \mathrm{S}$ expressing flies or flies expressing Lac-Z showed no degeneration compared to the expressing control flies. The results indicate that the 
fly system can be used to assess the capacity to form pre-fibrillar oligomers of $\alpha \mathrm{S}$ variants by the extent to which they cause neurodegenerative effects in the organism.

Most importantly, however, I have established the first animal PD model that can be used to study not only neurodegenerative processes but also non-motor symptoms known from PD patients. The available evidence suggests that the Drosophila PD model recapitulates sleep abnormalities and circadian deficits that are also observed with humans suffering from PD. I found that the circadian deficits observed in flies that express the most toxic TP- $\alpha \mathrm{S}$ variant are DA neuron-dependent effects as the genetic inactivation of DA neurons caused the same circadian deficits as observed after expression of the $\alpha \mathrm{S}$ mutation. Although we do not know whether the abnormal sleep behavior of the TP- $\alpha \mathrm{S}$ expressing flies is due to direct or indirect effects of the dysfunctional DA neurons, the sensitive and clearly graded effects on sleep correlates again with the moreoligomer-forming tendencies of the $\alpha \mathrm{S}$ variants used in the experiments. Therefore, not only neurodegeneration but also sleep abnormalities can be used as a sensitive readout to correlate the oligomer-forming tendency of $\alpha \mathrm{S}$ in vitro to the neurotoxicity in vivo. Given the conserved role of DA neuronal signaling in mammals and Drosophila, and protective effects of sleep on neurodegeneration, we can use the Drosophila model to even screen for therapeutic compounds for alleviating the sleep abnormalities in the human PD. I also found that the moreoligomer-forming $\alpha \mathrm{S}$ variants failed to localize to the synaptic terminals of the Drosophila larval NMJs whereas the WT- $\alpha \mathrm{S}$ localized properly to the synaptic terminals as observed in mammals. Whether the mislocalization of TP- $\alpha \mathrm{S}$ has any effect on synaptic function, which do not involve the glutamatergic system during the larval stage, and whether a possible effect is age-dependent remains to be shown.

Taken together, this work has shown that more-oligomer-forming $\alpha S$ variants are highly pathogenic. This observation strengthens the hypothesis that 
pre-fibrillar oligomers are a potent pathogenic $\alpha$ S species in PD and possibly also in other synucleinopathies. The severe pathogenic effects caused by the moreoligomer-forming $\alpha \mathrm{S}$ variants are also corroborated by the results from other animal models that were concurrently used to address the same question. Whether the pre-fibrillar oligomers are the primary cause of neurodegeneration or whether this process eventually requires $\alpha \mathrm{S}$ aggregation in addition to the toxic oligomers remains to be shown. However, in addition to supporting the hypothesis that the pre-fibrillar status of $\alpha \mathrm{S}$ is at least toxic for neurons, my results establish a novel experimental system in which the non-motor aspects of $\mathrm{PD}$, the mechanism of cellular action and likely even the efficacy of lead compounds leading to therapeutic drugs can be addressed in the future. 
A

\section{APPENDIX}

\section{Algorithms used for the sleep analysis in Drosophila}

The following 'source code' (SleepAnalysis.scr) was used for the sleep analyses carried out in this study. Running "SleepAnalysis.scr" (Script 1) elicits and runs three AWK programmes subsequently: sleepAnalysis.awk (Script 2), sleep30minRed.awk (Script 3), and outlierFly.awk (Script 4). Data collected from the Drosophila Activity Monitors (DAMs) in one-minute bins was used as an input for the programme. Output of this procedure gives a sleep-data matrix containing the defined sleep parameters in (see Figure 15, Section 3.8) for all the flies. All the comments used to explain the programming steps are preceded by the symbol '\#'.

\section{Script 1}

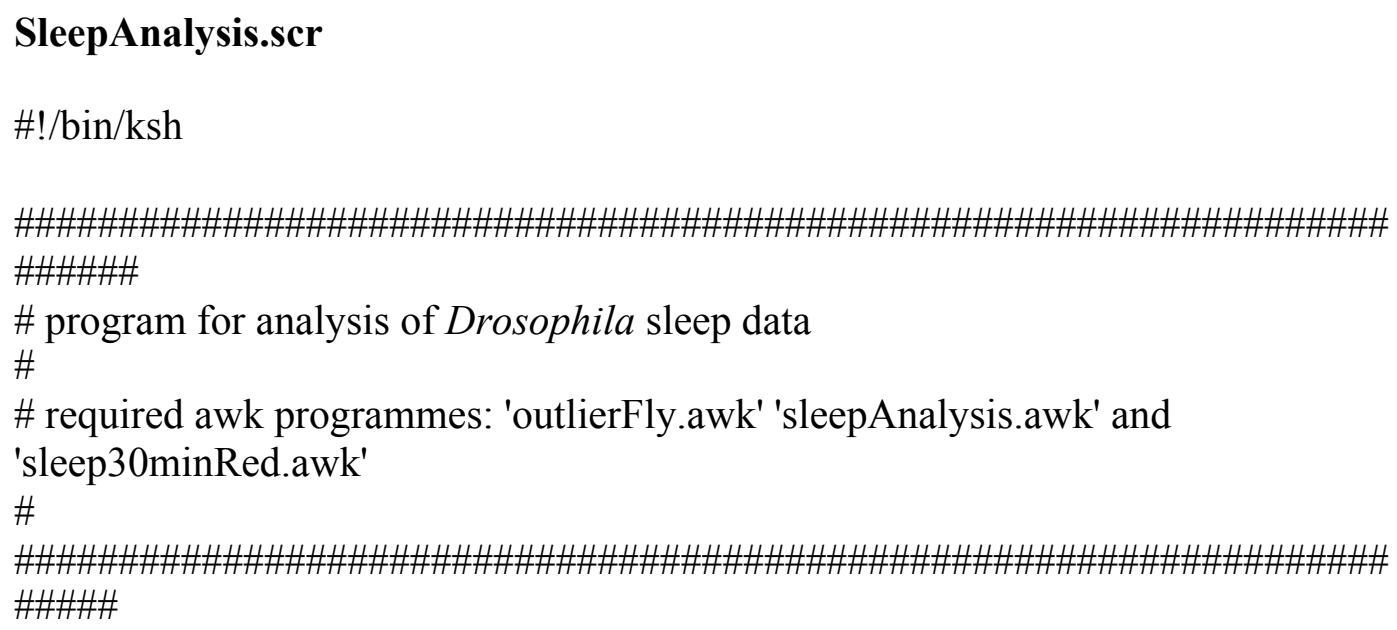




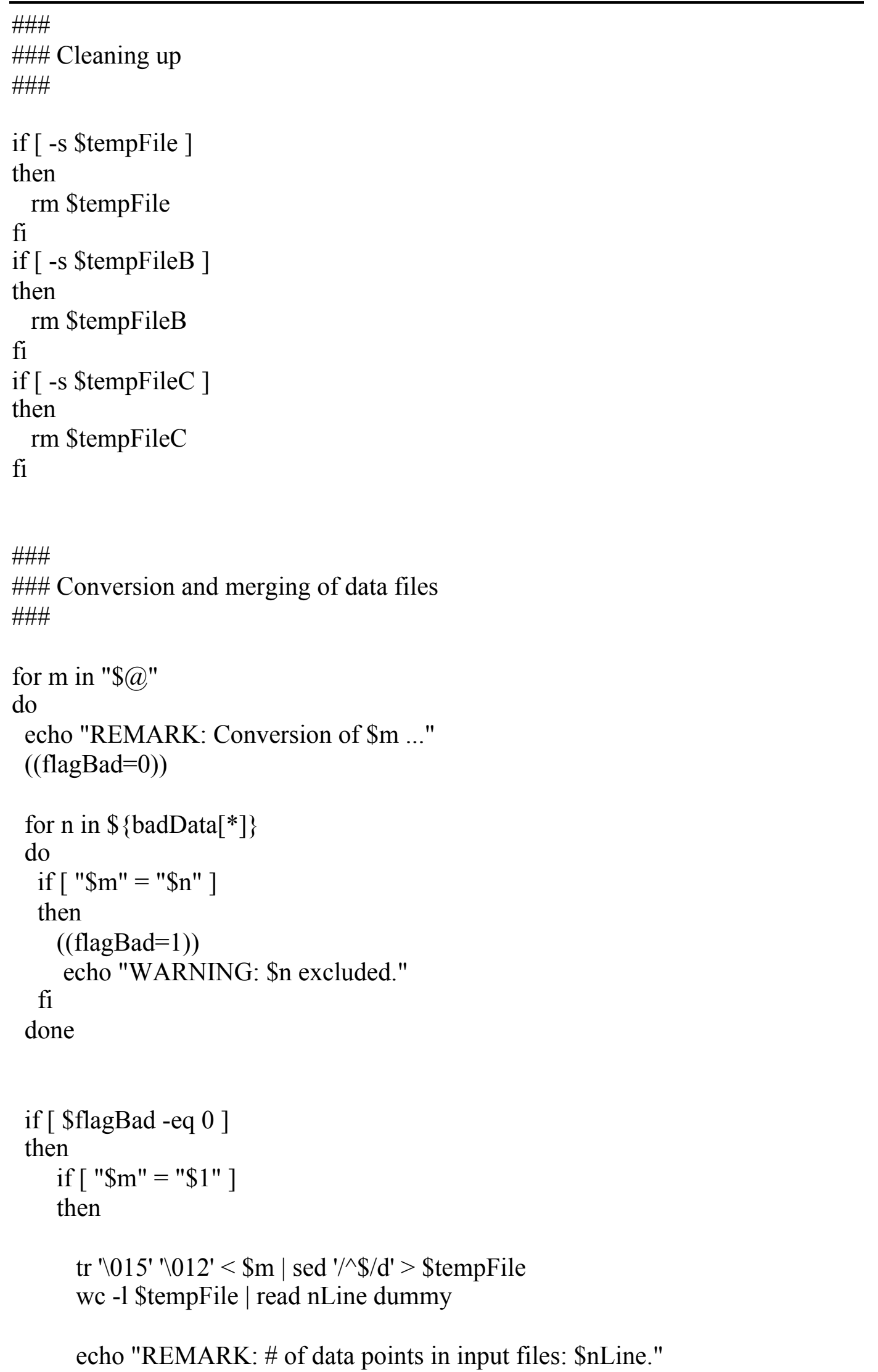


echo "REMARK: Conversion of first data file \$m successful." echo "'"

else

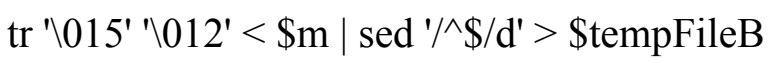

wc -1 \$tempFileB | read nLineB dummy

if [ \$nLine -eq \$nLineB ]

then

paste \$tempFile \$tempFileB > \$tempFileC

mv \$tempFileC \$tempFile

echo "REMARK: Conversion of \$m conversion successful." else

echo "WARNING: \$m does not have the same \# of lines."

echo "WARNING: \$m excluded from analysis."

exit

fi

fi

fi

echo ""

done

mv \$tempFile \$dataFile

\#\#\#

\#\#\# Selection of data points for analysis

\#\#\#

fgrep -n "\$startDate" \$dataFile | sed -e s/':'/' '/g | cut -d" " -f1 | read stLine

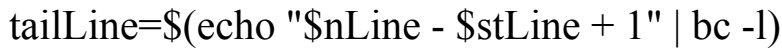

headLine $=\$($ echo "\$nHour * 60" | bc -1)

echo "REMARK: \$headLine data points selected. Starting date: \$startDate.

Duration: \$nHour hours."

echo $" n L i n e=\$ n L i n e ~ s t L i n e=\$$ stLine tailLine $=\$$ tailLine headLine $=\$$ headLine"

\#tail -\$tailLine \$dataFile | head -\$headLine > \$dataFileSel

\#nawk -v nL $=\$$ headLine $\mathrm{stL}=\$$ stLine 'BEGIN $\{\mathrm{i}=0 ; \mathrm{ok}=0\} ; \$ 1==$ "20080121" \& \& $\$ 2==$ "0000" $\{\mathrm{ok}=1 ; \mathrm{i}++\} ; \mathrm{ok}==1 \& \& \mathrm{i}<=\mathrm{nL}$ \{print $\$ 0 ; \mathrm{i}++\}$ ' $\$$ dataFile $>$ \$dataFileSel 
nawk $-\mathrm{v}$ nL $=\$$ headLine $-\mathrm{v}$ stL $=\$$ stLine 'BEGIN $\{\mathrm{i}=0 ; \mathrm{ok}=0\} ; \mathrm{NR}==\mathrm{stL}$ $\{\mathrm{ok}=1 ; \mathrm{i}++\} ; \mathrm{ok}==1 \& \& \mathrm{i}<=\mathrm{nL}$ \{print $\$ 0 ; \mathrm{i}++\}$ ' $\$$ dataFile $>$ \$dataFileSel

\author{
\#\#\# \\ \#\#\# Sleep analysis for each day \\ \#\#\# \\ nawk -f sleepAnalysis.awk \$dataFileSel > \$sleepFile
}

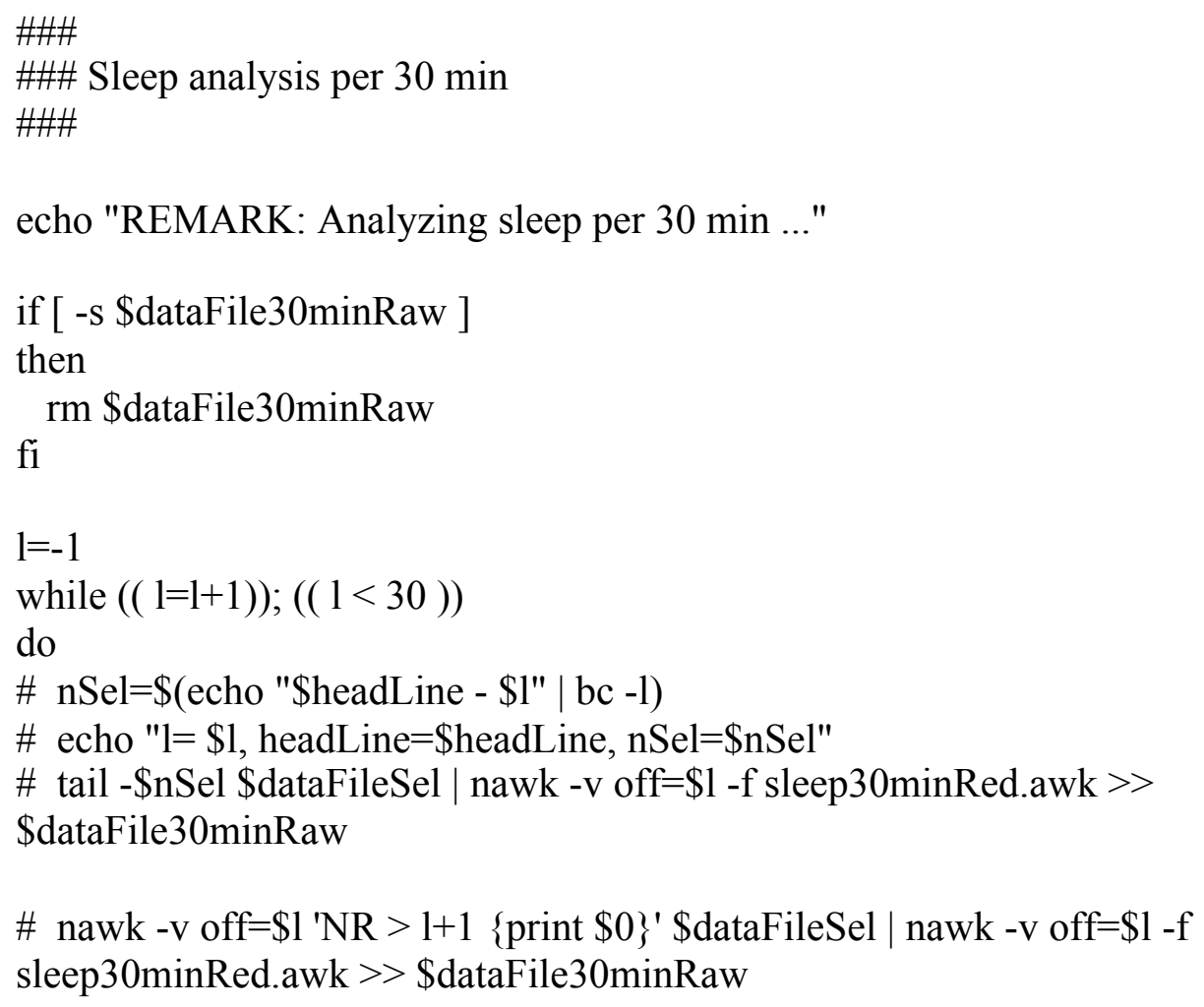




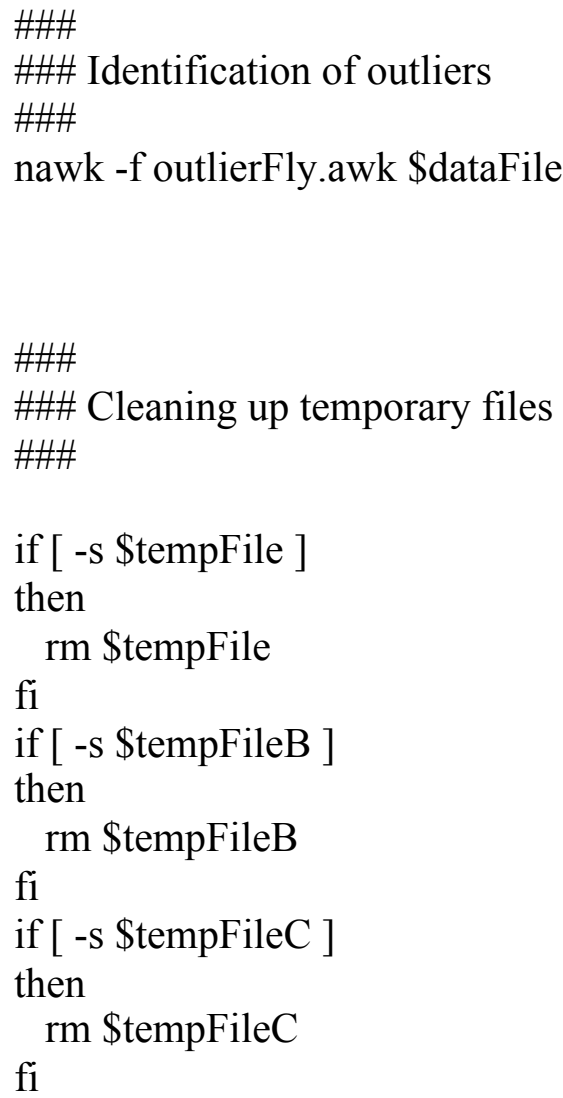




\section{Script 2}

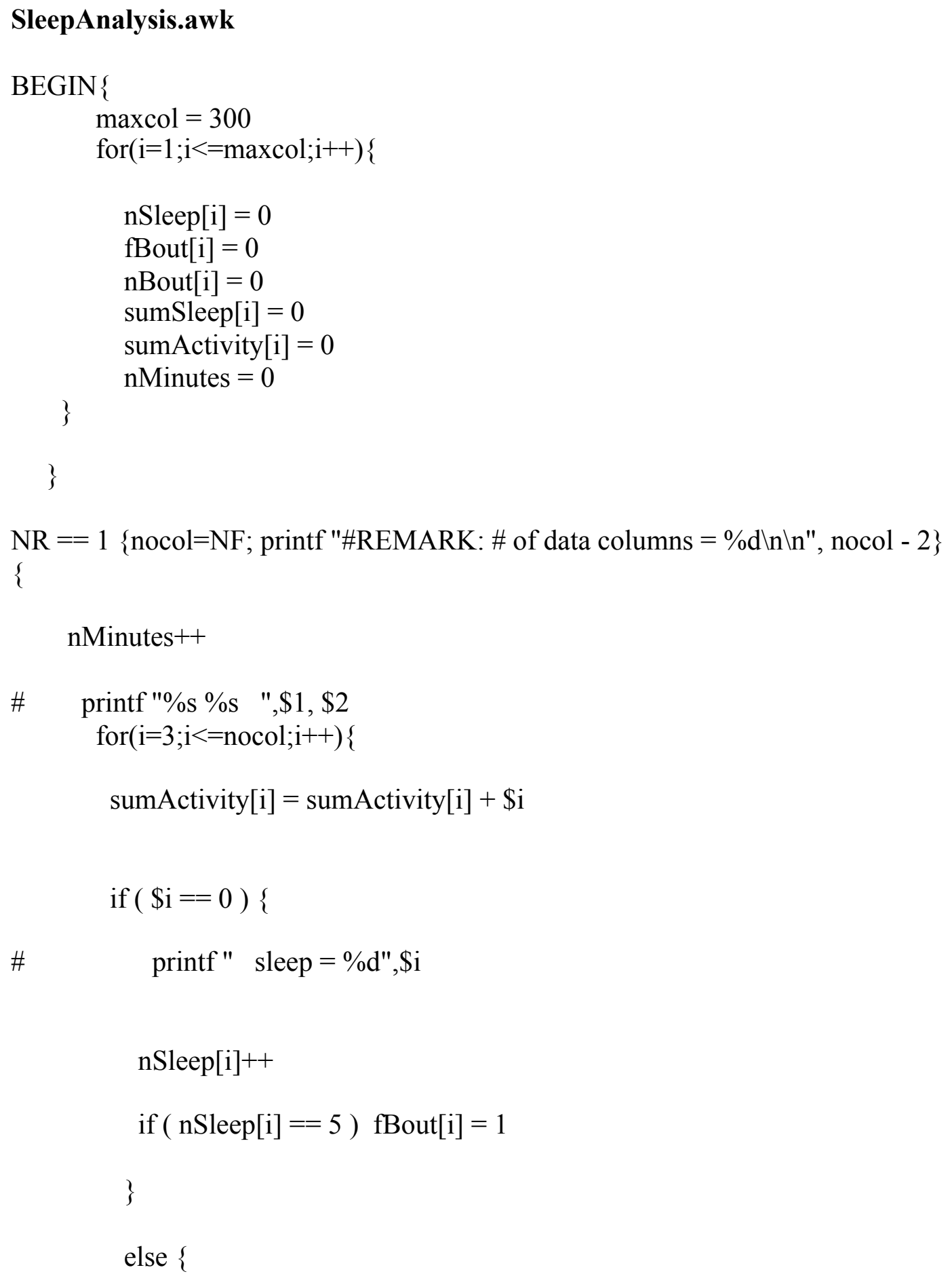




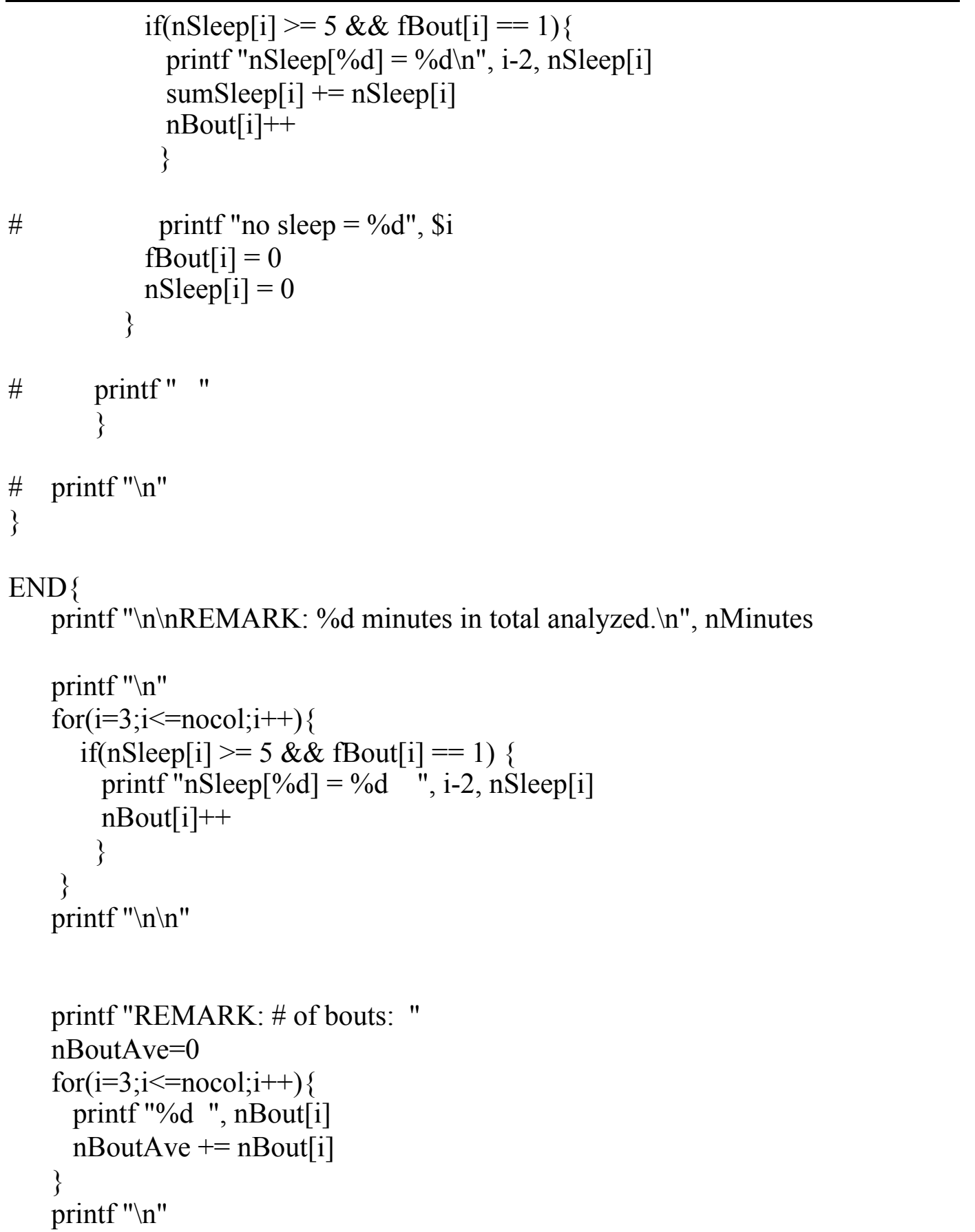

printf "REMARK: Average bout length: " lengthBoutAve $=0$

for $(\mathrm{i}=3 ; \mathrm{i}<=$ nocol $; \mathrm{i}++)\{$ printf "\%d ", sumSleep[i] / nBout[i] lengthBoutAve $+=$ sumSleep[i] / nBout[i] \}

printf " $\backslash n \backslash n "$ 
printf "REMARK: Total amount of sleep: "

sumSleepAve $=0$

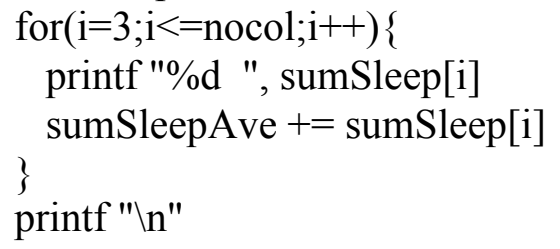

printf "REMARK: Average number of bouts for all flies $=\% .3 \mathrm{f} \backslash \mathrm{n} "$, nBoutAve / (nocol-2)

printf "REMARK: Average bout length for all flies $=\% .3 \mathrm{f} \backslash \mathrm{n}$ ", lengthBoutAve / (nocol-2)

printf "REMARK: Average of total amount of sleep for all flies $=\% .3 \mathrm{f} n$ ", sumSleepAve / (nocol-2) 
printf "REMARK: Average of total wake period for all flies $=\% .3 \mathrm{f} \backslash \mathrm{n}$ ", wakeAve / (nocol-2)

printf "REMARK: Average of total activity for all flies $=\% .3 \mathrm{fln} "$, sumActivityAve / (nocol-2)

printf "REMARK: Average of normalized total activity for all flies $=\% .3 \mathrm{f} \backslash \mathrm{n}$ ", normSumActivityAve / (nocol-2)

\} 


\section{Script 3}

\section{sleep30minRed.awk}

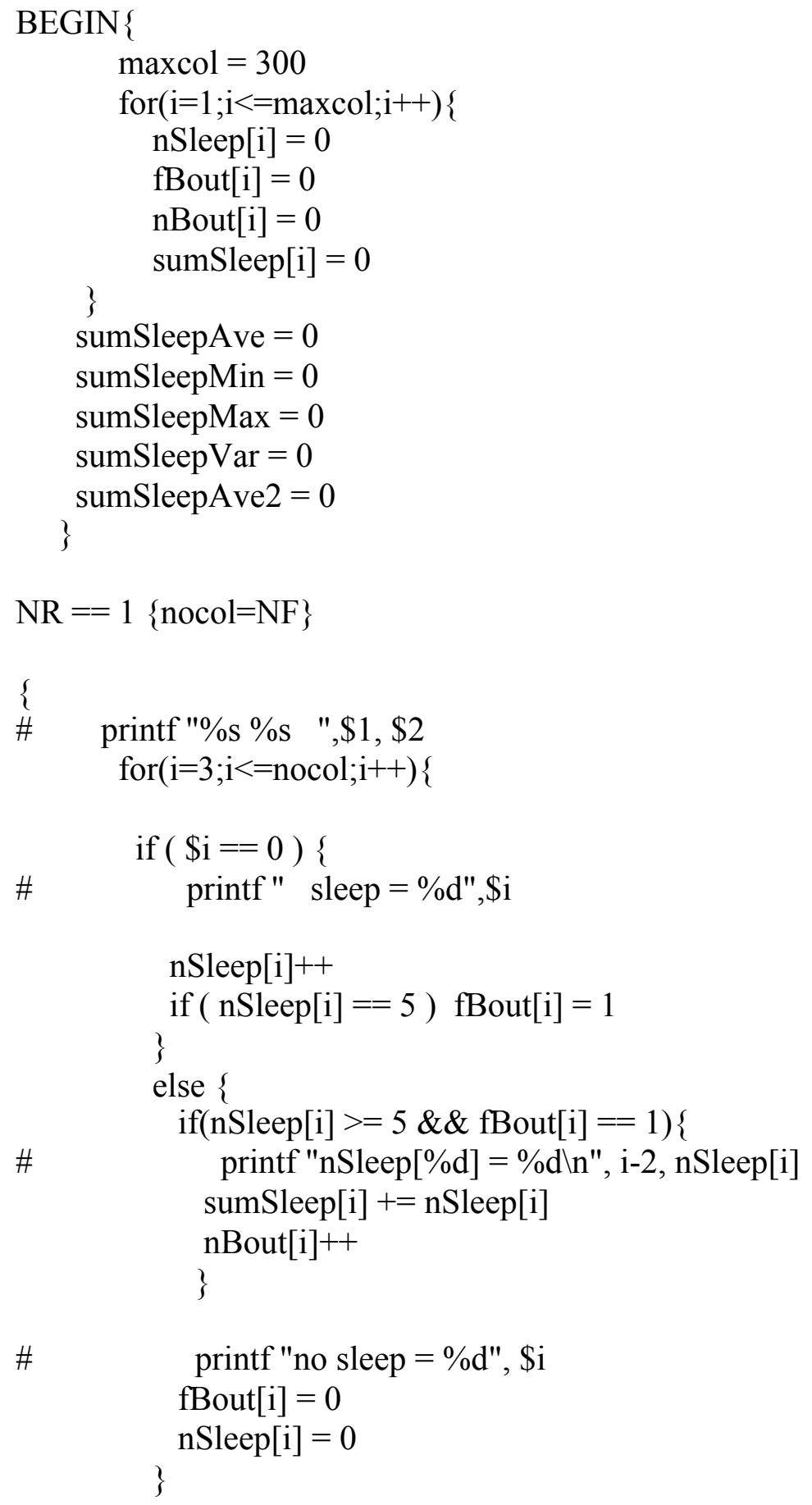




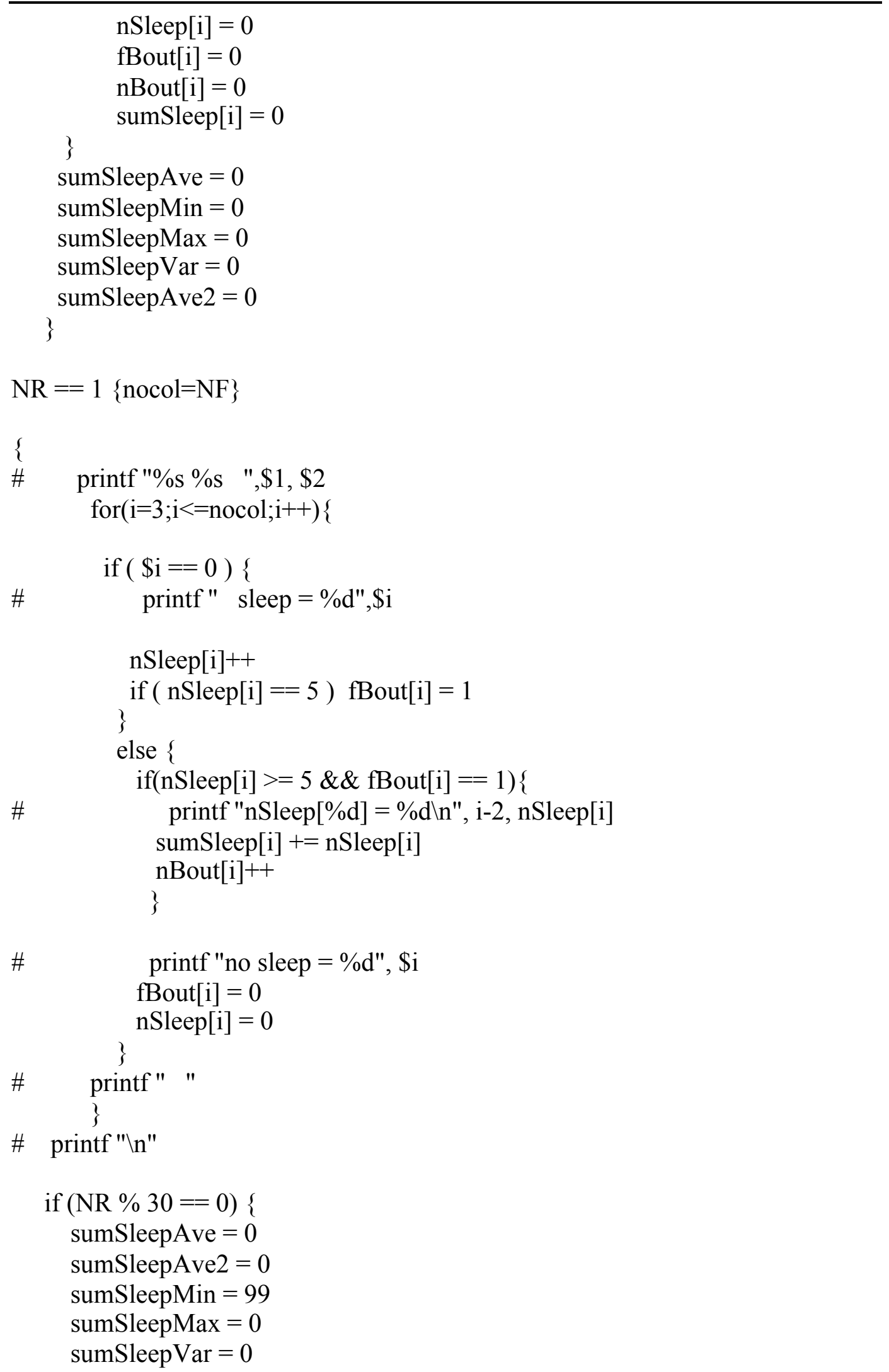




\section{Script 4}

\section{outlierFly.awk}

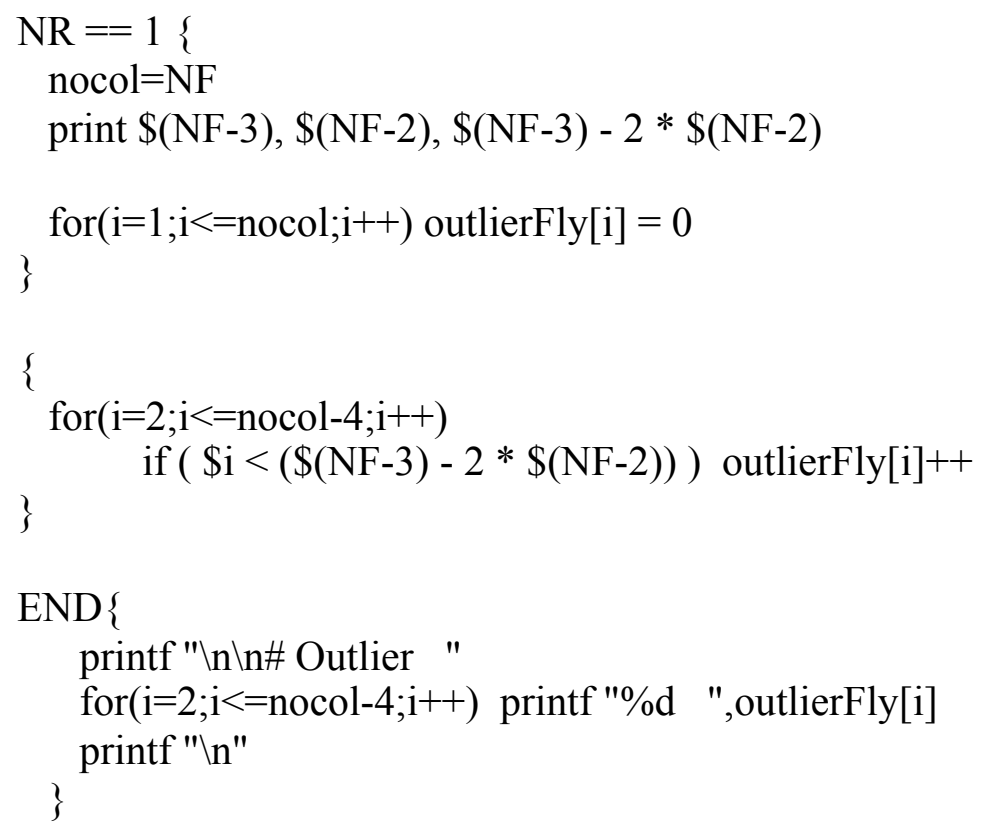


Figure 26: Circadian locomotor activity profiles of old Drosophila expressing WT$\alpha S$ and $A 53 T-\alpha S$

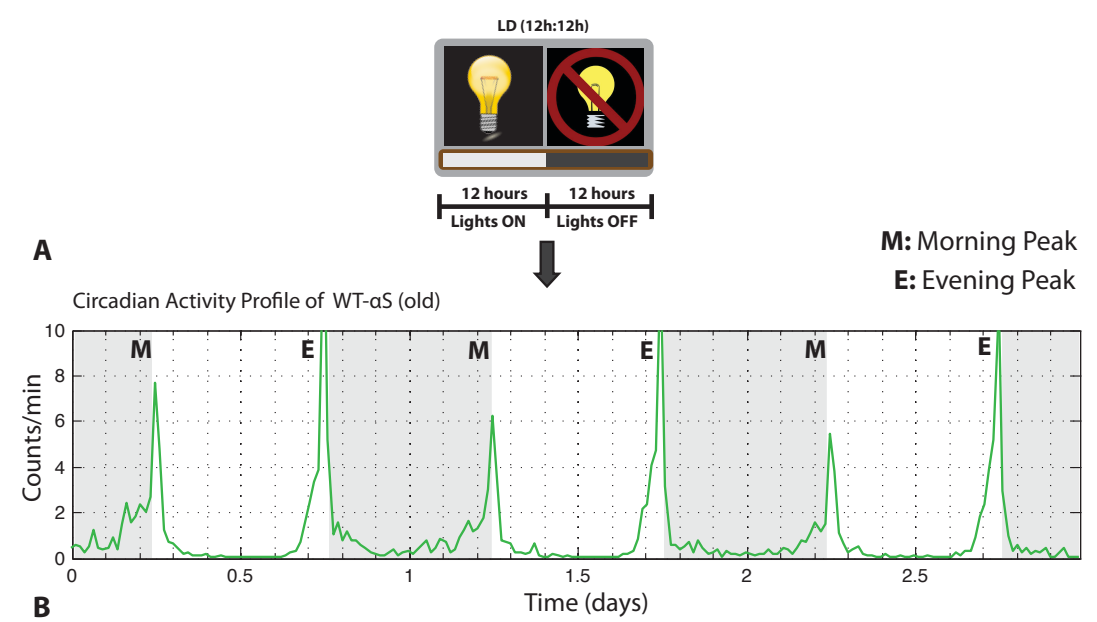

Circadian Activity Profile of lacZ (old))

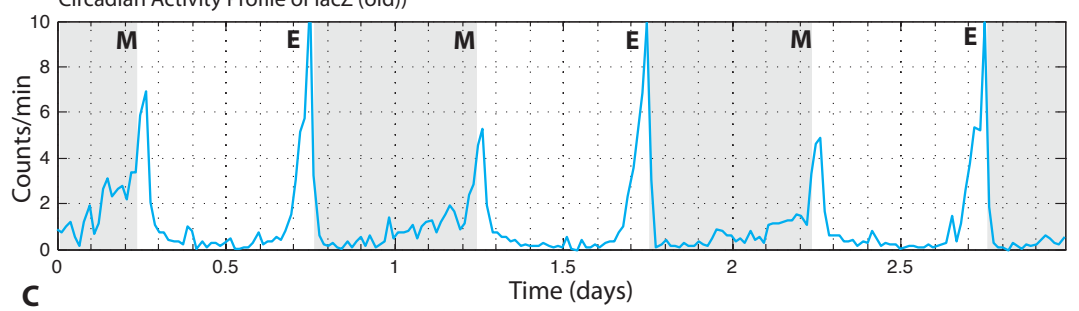

Circadian Activity Profile of A53T-aS (old))
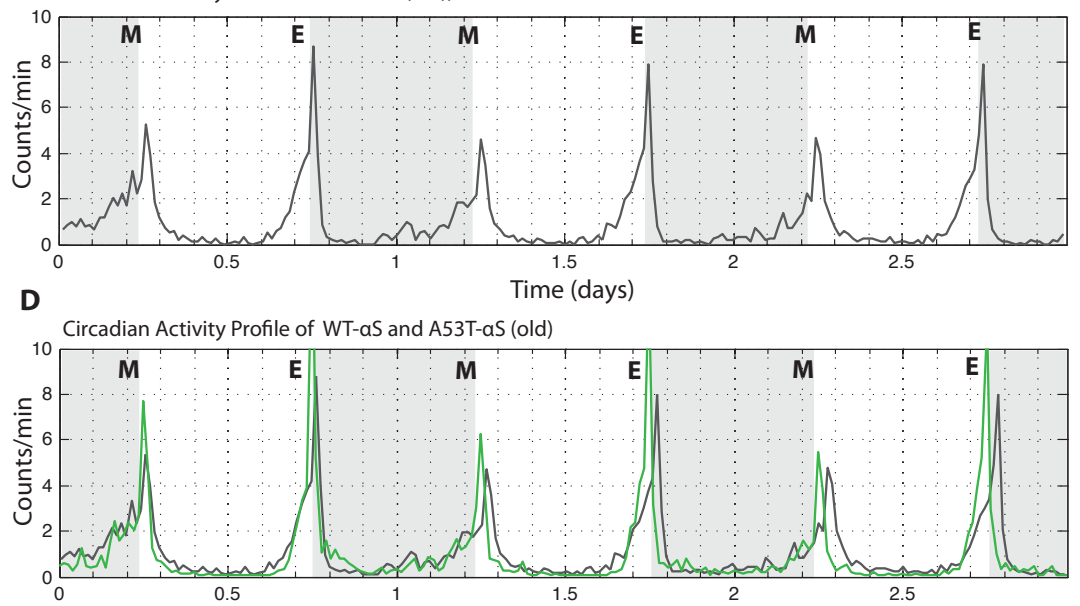

E

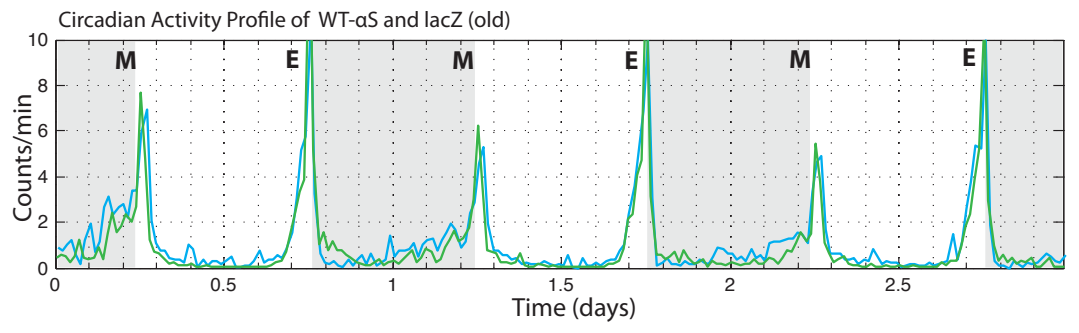


(A) Time-series circadian activity profile of the 30-day old flies expressing WT- $\alpha \mathrm{S}$ in DA neurons; $n=45$. (B) Time-series circadian activity profile of the 30-day old flies expressing lacZ in DA neurons; $n=28-32$. (C) Time-series circadian activity profile of the 30-day old flies expressing A53T- $\alpha \mathrm{S}$ in DA neurons; $n=32$. (D) Overlay of the activity profiles of WT- $\alpha \mathrm{S}$ (green) and A53T- $\alpha \mathrm{S}$ (grey). (E) Overlay of the activity profiles of WT- $\alpha S$ (green) and lacZ (blue). Experiments (Phase A according to the Figure 17) were done under the LD (12h: 12h) conditions. 


\section{BIBLIOGRAPHY}

Abeliovich, A., Schmitz, Y., Farinas, I., Choi-Lundberg, D., Ho, W.H., Castillo, P.E., Shinsky, N., Verdugo, J.M., Armanini, M., Ryan, A., et al. (2000). Mice lacking alpha-synuclein display functional deficits in the nigrostriatal dopamine system. Neuron 25, 239-252.

Abou-Sleiman, P.M., Muqit, M.M., and Wood, N.W. (2006). Expanding insights of mitochondrial dysfunction in Parkinson's disease. Nat Rev Neurosci 7, 207219.

Andretic, R., van Swinderen, B., and Greenspan, R.J. (2005). Dopaminergic modulation of arousal in Drosophila. Curr Biol 15, 1165-1175.

Antzutkin, O.N., Balbach, J.J., Leapman, R.D., Rizzo, N.W., Reed, J., and Tycko, R. (2000). Multiple quantum solid-state NMR indicates a parallel, not antiparallel, organization of beta-sheets in Alzheimer's beta-amyloid fibrils. Proc Natl Acad Sci U S A 97, 13045-13050.

Arnulf, I., Leu, S., and Oudiette, D. (2008). Abnormal sleep and sleepiness in Parkinson's disease. Curr Opin Neurol 21, 472-477.

Arrasate, M., Mitra, S., Schweitzer, E.S., Segal, M.R., and Finkbeiner, S. (2004). Inclusion body formation reduces levels of mutant huntingtin and the risk of neuronal death. Nature 431, 805-810.

Auluck, P.K., and Bonini, N.M. (2002). Pharmacological prevention of Parkinson disease in Drosophila. Nat Med 8, 1185-1186.

Auluck, P.K., Chan, H.Y., Trojanowski, J.Q., Lee, V.M., and Bonini, N.M. (2002). Chaperone suppression of alpha-synuclein toxicity in a Drosophila model for Parkinson's disease. Science 295, 865-868.

Ausubel, F.M. (1999). Current Protocols in Molecular Biology. John Wiley \& Sons, Inc. 
Baines, R.A., Uhler, J.P., Thompson, A., Sweeney, S.T., and Bate, M. (2001). Altered electrical properties in Drosophila neurons developing without synaptic transmission. J Neurosci 21, 1523-1531.

Bainton, R.J., Tsai, L.T., Singh, C.M., Moore, M.S., Neckameyer, W.S., and Heberlein, U. (2000). Dopamine modulates acute responses to cocaine, nicotine and ethanol in Drosophila. Curr Biol 10, 187-194.

Balbach, J.J., Petkova, A.T., Oyler, N.A., Antzutkin, O.N., Gordon, D.J., Meredith, S.C., and Tycko, R. (2002). Supramolecular structure in full-length Alzheimer's beta-amyloid fibrils: evidence for a parallel beta-sheet organization from solid-state nuclear magnetic resonance. Biophys J 83, 1205-1216.

Barrachina, M., Castano, E., Dalfo, E., Maes, T., Buesa, C., and Ferrer, I. (2006). Reduced ubiquitin C-terminal hydrolase-1 expression levels in dementia with Lewy bodies. Neurobiol Dis 22, 265-273.

Bernado, P., Bertoncini, C.W., Griesinger, C., Zweckstetter, M., and Blackledge, M. (2005). Defining long-range order and local disorder in native alpha-synuclein using residual dipolar couplings. J Am Chem Soc 127, 17968-17969.

Bertoncini, C.W., Jung, Y.-S., Fernandez, C.O., Hoyer, W., Griesinger, C., Jovin, T.M., and Zweckstetter, M. (2005). From The Cover: Release of long-range tertiary interactions potentiates aggregation of natively unstructured \{alpha\}synuclein. Proceedings of the National Academy of Sciences 102, 1430-1435.

Bischof, J., Maeda, R.K., Hediger, M., Karch, F., and Basler, K. (2007). An optimized transgenesis system for Drosophila using germ-line-specific phiC31 integrases. Proc Natl Acad Sci U S A 104, 3312-3317.

Braak, H., Del Tredici, K., Rub, U., de Vos, R.A., Jansen Steur, E.N., and Braak, E. (2003). Staging of brain pathology related to sporadic Parkinson's disease. Neurobiol Aging 24, 197-211.

Brand, A.H., and Perrimon, N. (1993). Targeted gene expression as a means of altering cell fates and generating dominant phenotypes. Development 118, 401415.

Bredesen, D.E., Rao, R.V., and Mehlen, P. (2006). Cell death in the nervous system. Nature 443, 796-802.

Broadie, K.S. (2000). In "Drosophila protocols" Cold Spring Harbor Laboratory Press 273-295. 
Cabin, D.E., Shimazu, K., Murphy, D., Cole, N.B., Gottschalk, W., McIlwain, K.L., Orrison, B., Chen, A., Ellis, C.E., Paylor, R., et al. (2002). Synaptic vesicle depletion correlates with attenuated synaptic responses to prolonged repetitive stimulation in mice lacking alpha-synuclein. J Neurosci 22, 8797-8807.

Catalina Ruiz-canada, V.B. (2006). The Fly Neuromuscular Junction: Structure and Function, Vol 75.

Caughey, B., and Lansbury, P.T. (2003). Protofibrils, pores, fibrils, and neurodegeneration: separating the responsible protein aggregates from the innocent bystanders. Annu Rev Neurosci 26, 267-298.

Chandra, S., Fornai, F., Kwon, H.B., Yazdani, U., Atasoy, D., Liu, X., Hammer, R.E., Battaglia, G., German, D.C., Castillo, P.E., et al. (2004). Double-knockout mice for alpha- and beta-synucleins: effect on synaptic functions. Proc Natl Acad Sci U S A 101, 14966-14971.

Chandra, S., Gallardo, G., Fernandez-Chacon, R., Schluter, O.M., and Sudhof, T.C. (2005). Alpha-synuclein cooperates with CSPalpha in preventing neurodegeneration. Cell 123, 383-396.

Chaudhuri, K.R., and Schapira, A.H. (2009). Non-motor symptoms of Parkinson's disease: dopaminergic pathophysiology and treatment. Lancet Neurol 8, 464-474.

Chen, L., and Feany, M.B. (2005). Alpha-synuclein phosphorylation controls neurotoxicity and inclusion formation in a Drosophila model of Parkinson disease. Nat Neurosci 8, 657-663.

Chen, L., Periquet, M., Wang, X., Negro, A., McLean, P.J., Hyman, B.T., and Feany, M.B. (2009). Tyrosine and serine phosphorylation of alpha-synuclein have opposing effects on neurotoxicity and soluble oligomer formation. J Clin Invest $119,3257-3265$.

Chien, S., Reiter, L.T., Bier, E., and Gribskov, M. (2002). Homophila: human disease gene cognates in Drosophila. Nucleic Acids Res 30, 149-151.

Chiti, F., and Dobson, C.M. (2006). Protein misfolding, functional amyloid, and human disease. Annu Rev Biochem 75, 333-366.

Cirelli, C., Bushey, D., Hill, S., Huber, R., Kreber, R., Ganetzky, B., and Tononi, G. (2005). Reduced sleep in Drosophila Shaker mutants. Nature 434, 1087-1092. 
Cirrito, J.R., Yamada, K.A., Finn, M.B., Sloviter, R.S., Bales, K.R., May, P.C., Schoepp, D.D., Paul, S.M., Mennerick, S., and Holtzman, D.M. (2005). Synaptic activity regulates interstitial fluid amyloid-beta levels in vivo. Neuron 48, 913922.

Clayton, D.F., and George, J.M. (1998). The synucleins: a family of proteins involved in synaptic function, plasticity, neurodegeneration and disease. Trends Neurosci 21, 249-254.

Colwell, C.S. (2007). Soporific signaling: how flies sleep through the night. Nat Neurosci 10, 1079-1080.

Conway, K.A., Harper, J.D., and Lansbury, P.T. (1998). Accelerated in vitro fibril formation by a mutant alpha-synuclein linked to early-onset Parkinson disease. Nat Med 4, 1318-1320.

Conway, K.A., Lee, S.J., Rochet, J.C., Ding, T.T., Williamson, R.E., and Lansbury, P.T., Jr. (2000). Acceleration of oligomerization, not fibrillization, is a shared property of both alpha-synuclein mutations linked to early-onset Parkinson's disease: implications for pathogenesis and therapy. Proc Natl Acad Sci U S A 97, 571-576.

Cookson, M.R. (2005). The biochemistry of Parkinson's disease. Annu Rev Biochem 74, 29-52.

Cooper, A.A., Gitler, A.D., Cashikar, A., Haynes, C.M., Hill, K.J., Bhullar, B., Liu, K., Xu, K., Strathearn, K.E., Liu, F., et al. (2006). Alpha-synuclein blocks ER-Golgi traffic and Rab1 rescues neuron loss in Parkinson's models. Science $313,324-328$.

Cummings, C.J., Reinstein, E., Sun, Y., Antalffy, B., Jiang, Y., Ciechanover, A., Orr, H.T., Beaudet, A.L., and Zoghbi, H.Y. (1999). Mutation of the E6-AP ubiquitin ligase reduces nuclear inclusion frequency while accelerating polyglutamine-induced pathology in SCA1 mice. Neuron 24, 879-892.

Dedmon, M. M., Lindorff-Larsen, K., Christodoulou, J., Vendruscolo, M. and Dobson, C. M (2005). Mapping Long-Range Interactions in $\alpha$-Synuclein using Spin-Label NMR and Ensemble Molecular Dynamics Simulations J. Am. Chem. Soc., 127, 476- 7 .

Dickson, D.W., Crystal, H.A., Mattiace, L.A., Masur, D.M., Blau, A.D., Davies, P., Yen, S.H., and Aronson, M.K. (1992). Identification of normal and 
pathological aging in prospectively studied nondemented elderly humans. Neurobiol Aging 13, 179-189.

Ding, T.T., Lee, S.J., Rochet, J.C., and Lansbury, P.T., Jr. (2002). Annular alphasynuclein protofibrils are produced when spherical protofibrils are incubated in solution or bound to brain-derived membranes. Biochemistry 41, 10209-10217.

Dobson, C.M. (2003). Protein folding and misfolding. Nature 426, 884-890.

Dzirasa, K., Ribeiro, S., Costa, R., Santos, L.M., Lin, S.C., Grosmark, A., Sotnikova, T.D., Gainetdinov, R.R., Caron, M.G., and Nicolelis, M.A. (2006). Dopaminergic control of sleep-wake states. J Neurosci 26, 10577-10589.

Ebstein, R.P., Israel, S., Chew, S.H., Zhong, S., and Knafo, A. (2010). Genetics of human social behavior. Neuron 65, 831-844.

Ehrnhoefer, D.E., Bieschke, J., Boeddrich, A., Herbst, M., Masino, L., Lurz, R., Engemann, S., Pastore, A., and Wanker, E.E. (2008). EGCG redirects amyloidogenic polypeptides into unstructured, off-pathway oligomers. Nat Struct Mol Biol 15, 558-566.

El-Agnaf, O.M., Jakes, R., Curran, M.D., Middleton, D., Ingenito, R., Bianchi, E., Pessi, A., Neill, D., and Wallace, A. (1998). Aggregates from mutant and wildtype alpha-synuclein proteins and NAC peptide induce apoptotic cell death in human neuroblastoma cells by formation of beta-sheet and amyloid-like filaments. FEBS Lett 440, 71-75.

Factor, S.A. (2002). Parkinson's Disease: Diagnosis and Clinical Management.

Fall, P.A., Saleh, A., Fredrickson, M., Olsson, J.E., and Granerus, A.K. (2003). Survival time, mortality, and cause of death in elderly patients with Parkinson's disease: a 9-year follow-up. Mov Disord 18, 1312-1316.

Farrer, M.J. (2006). Genetics of Parkinson disease: paradigm shifts and future prospects. Nat Rev Genet 7, 306-318.

Feany, M.B., and Bender, W.W. (2000). A Drosophila model of Parkinson's disease. Nature 404, 394-398.

Foltenyi, K., Greenspan, R.J., and Newport, J.W. (2007). Activation of EGFR and ERK by rhomboid signaling regulates the consolidation and maintenance of sleep in Drosophila. Nat Neurosci 10, 1160-1167. 
Forman, M.S., Trojanowski, J.Q., and Lee, V.M. (2004). Neurodegenerative diseases: a decade of discoveries paves the way for therapeutic breakthroughs. Nat Med 10, 1055-1063.

Friggi-Grelin, F., Coulom, H., Meller, M., Gomez, D., Hirsh, J., and Birman, S. (2003). Targeted gene expression in Drosophila dopaminergic cells using regulatory sequences from tyrosine hydroxylase. J Neurobiol 54, 618-627.

Fujiwara, H., Hasegawa, M., Dohmae, N., Kawashima, A., Masliah, E., Goldberg, M.S., Shen, J., Takio, K., and Iwatsubo, T. (2002). alpha-Synuclein is phosphorylated in synucleinopathy lesions. Nat Cell Biol 4, 160-164.

George, J.M., Jin, H., Woods, W.S., and Clayton, D.F. (1995). Characterization of a novel protein regulated during the critical period for song learning in the zebra finch. Neuron 15, 361-372.

Gitler, A.D., Bevis, B.J., Shorter, J., Strathearn, K.E., Hamamichi, S., Su, L.J., Caldwell, K.A., Caldwell, G.A., Rochet, J.C., McCaffery, J.M., et al. (2008). The Parkinson's disease protein alpha-synuclein disrupts cellular Rab homeostasis. Proc Natl Acad Sci U S A 105, 145-150.

Goedert, M. (2001). Alpha-synuclein and neurodegenerative diseases. Nat Rev Neurosci 2, 492-501.

Gosavi, N., Lee, H.J., Lee, J.S., Patel, S., and Lee, S.J. (2002). Golgi fragmentation occurs in the cells with prefibrillar alpha-synuclein aggregates and precedes the formation of fibrillar inclusion. J Biol Chem 277, 48984-48992.

Greenbaum, E.A., Graves, C.L., Mishizen-Eberz, A.J., Lupoli, M.A., Lynch, D.R., Englander, S.W., Axelsen, P.H., and Giasson, B.I. (2005). The E46K mutation in alpha-synuclein increases amyloid fibril formation. J Biol Chem 280, 7800-7807.

Haass, C., and Selkoe, D.J. (2007). Soluble protein oligomers in neurodegeneration: lessons from the Alzheimer's amyloid beta-peptide. Nat Rev Mol Cell Biol 8, 101-112.

Harbison, S.T., Mackay, T.F., and Anholt, R.R. (2009). Understanding the neurogenetics of sleep: progress from Drosophila. Trends Genet 25, 262-269.

Hardy, J., and Selkoe, D.J. (2002). The amyloid hypothesis of Alzheimer's disease: progress and problems on the road to therapeutics. Science 297, 353-356. 
Hartley, D.M., Walsh, D.M., Ye, C.P., Diehl, T., Vasquez, S., Vassilev, P.M., Teplow, D.B., and Selkoe, D.J. (1999). Protofibrillar intermediates of amyloid beta-protein induce acute electrophysiological changes and progressive neurotoxicity in cortical neurons. J Neurosci 19, 8876-8884.

Heise, H., Hoyer, W., Becker, S., Andronesi, O.C., Riedel, D., and Baldus, M. (2005a). Molecular-level secondary structure, polymorphism, and dynamics of full-length \{alpha\}-synuclein fibrils studied by solid-state NMR. Proceedings of the National Academy of Sciences 102, 15871-15876.

Heise, H., Hoyer, W., Becker, S., Andronesi, O.C., Riedel, D., and Baldus, M. (2005b). Molecular-level secondary structure, polymorphism, and dynamics of full-length alpha-synuclein fibrils studied by solid-state NMR. Proc Natl Acad Sci U S A 102, 15871-15876.

Hendricks, J.C., and Sehgal, A. (2004). Why a fly? Using Drosophila to understand the genetics of circadian rhythms and sleep. Sleep 27, 334-342.

Hirsh, J., Riemensperger, T., Coulom, H., Iche, M., Coupar, J., and Birman, S. (2010). Roles of dopamine in circadian rhythmicity and extreme light sensitivity of circadian entrainment. Curr Biol 20, 209-214.

Ho, K.S., and Sehgal, A. (2005). Drosophila melanogaster: An Insect Model for Fundamental Studies of Sleep. Methods Enzymol 393, 772-793.

Hsiao, K., and Prusiner, S.B. (1991). Molecular genetics and transgenic model of Gertsmann-Straussler-Scheinker disease. Alzheimer Dis Assoc Disord 5, 155-162.

Huber, R., Hill, S.L., Holladay, C., Biesiadecki, M., Tononi, G., and Cirelli, C. (2004). Sleep homeostasis in Drosophila melanogaster. Sleep 27, 628-639.

Ibanez, P., Bonnet, A.M., Debarges, B., Lohmann, E., Tison, F., Pollak, P., Agid, Y., Durr, A., and Brice, A. (2004). Causal relation between alpha-synuclein gene duplication and familial Parkinson's disease. Lancet 364, 1169-1171.

Iwai, A., Masliah, E., Yoshimoto, M., Ge, N., Flanagan, L., de Silva, H.A., Kittel, A., and Saitoh, T. (1995). The precursor protein of non-A beta component of Alzheimer's disease amyloid is a presynaptic protein of the central nervous system. Neuron 14, 467-475.

Jahn, R., Schiebler, W., and Greengard, P. (1984). A quantitative dotimmunobinding assay for proteins using nitrocellulose membrane filters. Proc Natl Acad Sci U S A 81, 1684-1687. 
Johnson, P.M., and Kenny, P.J. (2010). Dopamine D2 receptors in addiction-like reward dysfunction and compulsive eating in obese rats. Nat Neurosci.

Joiner, W.J., Crocker, A., White, B.H., and Sehgal, A. (2006). Sleep in Drosophila is regulated by adult mushroom bodies. Nature 441, 757-760.

Kahle, P.J., Neumann, M., Ozmen, L., Muller, V., Jacobsen, H., Schindzielorz, A., Okochi, M., Leimer, U., van Der Putten, H., Probst, A., et al. (2000). Subcellular localization of wild-type and Parkinson's disease-associated mutant alpha -synuclein in human and transgenic mouse brain. J Neurosci 20,6365-6373.

Karpinar, D.P., Balija, M.B., Kugler, S., Opazo, F., Rezaei-Ghaleh, N., Wender, N., Kim, H.Y., Taschenberger, G., Falkenburger, B.H., Heise, H., et al. (2009). Pre-fibrillar alpha-synuclein variants with impaired beta-structure increase neurotoxicity in Parkinson's disease models. EMBO J 28, 3256-3268.

Katzenschlager, R., Evans, A., Manson, A., Patsalos, P.N., Ratnaraj, N., Watt, H., Timmermann, L., Van der Giessen, R., and Lees, A.J. (2004). Mucuna pruriens in Parkinson's disease: a double blind clinical and pharmacological study. J Neurol Neurosurg Psychiatry 75, 1672-1677.

Kayed, R., Head, E., Thompson, J.L., McIntire, T.M., Milton, S.C., Cotman, C.W., and Glabe, C.G. (2003). Common structure of soluble amyloid oligomers implies common mechanism of pathogenesis. Science 300, 486-489.

Kim, H.Y., Cho, M.K., Kumar, A., Maier, E., Siebenhaar, C., Becker, S., Fernandez, C.O., Lashuel, H.A., Benz, R., Lange, A., et al. (2009). Structural properties of pore-forming oligomers of alpha-synuclein. J Am Chem Soc 131, 17482-17489.

Kim, Y.C., Lee, H.G., and Han, K.A. (2007). D1 dopamine receptor dDA1 is required in the mushroom body neurons for aversive and appetitive learning in Drosophila. J Neurosci 27, 7640-7647.

Kitada, T., Asakawa, S., Hattori, N., Matsumine, H., Yamamura, Y., Minoshima, S., Yokochi, M., Mizuno, Y., and Shimizu, N. (1998). Mutations in the parkin gene cause autosomal recessive juvenile parkinsonism. Nature 392, 605-608.

Kruger, R., Kuhn, W., Muller, T., Woitalla, D., Graeber, M., Kosel, S., Przuntek, H., Epplen, J.T., Schols, L., and Riess, O. (1998). Ala30Pro mutation in the gene encoding alpha-synuclein in Parkinson's disease. Nat Genet 18, 106-108. 
Kume, K., Kume, S., Park, S.K., Hirsh, J., and Jackson, F.R. (2005). Dopamine is a regulator of arousal in the fruit fly. J Neurosci 25, 7377-7384.

Lansbury, P.T., Jr., and Brice, A. (2002). Genetics of Parkinson's disease and biochemical studies of implicated gene products. Curr Opin Cell Biol 14, 653660 .

Lansbury, P.T., and Lashuel, H.A. (2006). A century-old debate on protein aggregation and neurodegeneration enters the clinic. Nature 443, 774-779.

Lashuel, H. A., Hartley, D., Petre, B. M., Walz, T. and Lansbury, P. T., Jr. (2002). NACP, a protein implicated in Alzheimer's disease and learning, is natively unfolded. Nature 418, 291.

Lashuel, H. A., Petre, B. M., Wall, J., Simon, M., Nowak, R. J., Walz, T. and Lansbury, P. T., Jr. J. Mol. Biol. 2002

Lashuel, H.A., and Lansbury, P.T., Jr. (2006). Are amyloid diseases caused by protein aggregates that mimic bacterial pore-forming toxins? Q Rev Biophys 39, 167-201.

Lee, V.M., and Trojanowski, J.Q. (2006). Mechanisms of Parkinson's disease linked to pathological alpha-synuclein: new targets for drug discovery. Neuron 52, 33-38.

Leroy, E., Boyer, R., Auburger, G., Leube, B., Ulm, G., Mezey, E., Harta, G., Brownstein, M.J., Jonnalagada, S., Chernova, T., et al. (1998). The ubiquitin pathway in Parkinson's disease. Nature 395, 451-452.

Lessing, D., and Bonini, N.M. (2009). Maintaining the brain: insight into human neurodegeneration from Drosophila melanogaster mutants. Nat Rev Genet 10, 359-370.

Lindsley, D.L., and Zimm, G.G. (1992). The Genome of Drosophila melanogaster. Academic Press.

Liu, Z., Wang, X., Li X, Wang, T., Jiang, H., Ren, Q., Jiao, Y., Sawa, A., Moran,T., Ross, C.A., Montell, C., Smith, W.W. (2008). A Drosophila model for LRRK2-linked parkinsonism. Proc Natl Acad Sci U S A 105, 2693-8.

Luan, H., Lemon, W.C., Peabody, N.C., Pohl, J.B., Zelensky, P.K., Wang, D., Nitabach, M.N., Holmes, T.C., and White, B.H. (2006). Functional dissection of a 
neuronal network required for cuticle tanning and wing expansion in Drosophila. J Neurosci 26, 573-584.

Manyam, B.V. (1990). Paralysis agitans and levodopa in "Ayurveda": ancient Indian medical treatise. Mov Disord 5, 47-48.

Maries, E., Dass, B., Collier, T.J., Kordower, J.H., and Steece-Collier, K. (2003). The role of alpha-synuclein in Parkinson's disease: insights from animal models. Nat Rev Neurosci 4, 727-738.

Maroteaux, L., Campanelli, J.T., and Scheller, R.H. (1988). Synuclein: a neuronspecific protein localized to the nucleus and presynaptic nerve terminal. $J$ Neurosci 8, 2804-2815.

Moore, D.J., West, A.B., Dawson, V.L., and Dawson, T.M. (2005). Molecular pathophysiology of Parkinson's disease. Annu Rev Neurosci 28, 57-87.

Mueller, J.C., Fuchs, J., Hofer, A., Zimprich, A., Lichtner, P., Illig, T., Berg, D., Wullner, U., Meitinger, T., and Gasser, T. (2005). Multiple regions of alphasynuclein are associated with Parkinson's disease. Ann Neurol 57, 535-541.

Myhre, R., Toft, M., Kachergus, J., Hulihan, M.M., Aasly, J.O., Klungland, H., and Farrer, M.J. (2008). Multiple alpha-synuclein gene polymorphisms are associated with Parkinson's disease in a Norwegian population. Acta Neurol Scand 118, 320-327.

Nawathean, P., and Rosbash, M. (2004). The doubletime and CKII kinases collaborate to potentiate Drosophila PER transcriptional repressor activity. Mol Cell 13, 213-223.

Nemani, V.M., Lu, W., Berge, V., Nakamura, K., Onoa, B., Lee, M.K., Chaudhry, F.A., Nicoll, R.A., and Edwards, R.H. (2010). Increased expression of alphasynuclein reduces neurotransmitter release by inhibiting synaptic vesicle reclustering after endocytosis. Neuron 65, 66-79.

Outeiro, T.F., Kontopoulos, E., Altmann, S.M., Kufareva, I., Strathearn, K.E., Amore, A.M., Volk, C.B., Maxwell, M.M., Rochet, J.C., McLean, P.J., et al. (2007). Sirtuin 2 inhibitors rescue alpha-synuclein-mediated toxicity in models of Parkinson's disease. Science 317, 516-519.

Outeiro, T.F., and Lindquist, S. (2003). Yeast cells provide insight into alphasynuclein biology and pathobiology. Science 302, 1772-1775. 
Pallier, P.N., Maywood, E.S., Zheng, Z., Chesham, J.E., Inyushkin, A.N., Dyball, R., Hastings, M.H., and Morton, A.J. (2007). Pharmacological imposition of sleep slows cognitive decline and reverses dysregulation of circadian gene expression in a transgenic mouse model of Huntington's disease. J Neurosci 27, 7869-7878.

Periquet, M., Fulga, T., Myllykangas, L., Schlossmacher, M.G., and Feany, M.B. (2007). Aggregated alpha-synuclein mediates dopaminergic neurotoxicity in vivo. J Neurosci 27, 3338-3346.

Perutz, M.F., and Windle, A.H. (2001). Cause of neural death in neurodegenerative diseases attributable to expansion of glutamine repeats. Nature $412,143-144$.

Petkova, A.T., Ishii, Y., Balbach, J.J., Antzutkin, O.N., Leapman, R.D., Delaglio, F., and Tycko, R. (2002). A structural model for Alzheimer's beta -amyloid fibrils based on experimental constraints from solid state NMR. Proc Natl Acad Sci U S A $99,16742-16747$.

Pitman, J.L., McGill, J.J., Keegan, K.P., and Allada, R. (2006). A dynamic role for the mushroom bodies in promoting sleep in Drosophila. Nature 441, 753-756.

Poewe, W. (2008). Non-motor symptoms in Parkinson's disease. Eur J Neurol 15 Suppl 1, 14-20.

Polymeropoulos, M.H., Lavedan, C., Leroy, E., Ide, S.E., Dehejia, A., Dutra, A., Pike, B., Root, H., Rubenstein, J., Boyer, R., et al. (1997). Mutation in the alphasynuclein gene identified in families with Parkinson's disease. Science 276, 20452047.

Pountney, D.L., Lowe, R., Quilty, M., Vickers, J.C., Voelcker, N.H., and Gai, W.P. (2004). Annular alpha-synuclein species from purified multiple system atrophy inclusions. J Neurochem 90, 502-512.

Qin, G., Schwarz, T., Kittel, R.J., Schmid, A., Rasse, T.M., Kappei, D., Ponimaskin, E., Heckmann, M., and Sigrist, S.J. (2005). Four different subunits are essential for expressing the synaptic glutamate receptor at neuromuscular junctions of Drosophila. J Neurosci 25, 3209-3218.

Rajput, A.H., Uitti, R.J., Rajput, A., and Offord, K.P. (2009). Mortality in Parkinson's disease. Mov Disord.

Reddy, A.B., and O'Neill, J.S. (2010). Healthy clocks, healthy body, healthy mind. Trends Cell Biol 20,36-44. 
Rochet, J.C., Conway, K.A., and Lansbury, P.T., Jr. (2000). Inhibition of fibrillization and accumulation of prefibrillar oligomers in mixtures of human and mouse alpha-synuclein. Biochemistry 39, 10619-10626.

Rosato, E., and Kyriacou, C.P. (2006). Analysis of locomotor activity rhythms in Drosophila. Nat Protoc 1, 559-568.

Rosen, A.D. (1978). Amyotrophic lateral sclerosis. Clinical features and prognosis. Arch Neurol 35, 638-642.

Ross, C.A., and Poirier, M.A. (2004). Protein aggregation and neurodegenerative disease. Nat Med 10 Suppl, S10-17.

Rubin, G.M., and Spradling, A.C. (1982). Genetic transformation of Drosophila with transposable element vectors. Science 218, 348-353.

Rubinsztein, D.C. (2006). The roles of intracellular protein-degradation pathways in neurodegeneration. Nature 443, 780-786.

Rye, D.B. (2006). Excessive daytime sleepiness and unintended sleep in Parkinson's disease. Curr Neurol Neurosci Rep 6, 169-176.

Sanchez, I., Mahlke, C., and Yuan, J. (2003). Pivotal role of oligomerization in expanded polyglutamine neurodegenerative disorders. Nature 421, 373-379.

Sang, T.K., Chang, H.Y., Lawless, G.M., Ratnaparkhi, A., Mee, L., Ackerson, L.C., Maidment, N.T., Krantz, D.E., and Jackson, G.R. (2007). A Drosophila model of mutant human parkin-induced toxicity demonstrates selective loss of dopaminergic neurons and dependence on cellular dopamine. J Neurosci 27, 981992.

Schaffar, G., Breuer, P., Boteva, R., Behrends, C., Tzvetkov, N., Strippel, N., Sakahira, H., Siegers, K., Hayer-Hartl, M., and Hartl, F.U. (2004). Cellular toxicity of polyglutamine expansion proteins: mechanism of transcription factor deactivation. Mol Cell 15, 95-105.

Schagger, H., and von Jagow, G. (1987). Tricine-sodium dodecyl sulfatepolyacrylamide gel electrophoresis for the separation of proteins in the range from 1 to $100 \mathrm{kDa}$. Anal Biochem 166, 368-379.

Schapira, A.H. (2004). Excessive daytime sleepiness in Parkinson's disease. Neurology 63, S24-27. 
Selkoe, D.J. (2003). Folding proteins in fatal ways. Nature 426, 900-904.

Seugnet, L., Galvin, J.E., Suzuki, Y., Gottschalk, L., and Shaw, P.J. (2009). Persistent short-term memory defects following sleep deprivation in a drosophila model of Parkinson disease. Sleep 32, 984-992.

Sharon, R., Bar-Joseph, I., Frosch, M.P., Walsh, D.M., Hamilton, J.A., and Selkoe, D.J. (2003a). The formation of highly soluble oligomers of alphasynuclein is regulated by fatty acids and enhanced in Parkinson's disease. Neuron 37, 583-595.

Sharon, R., Bar-Joseph, I., Mirick, G.E., Serhan, C.N., and Selkoe, D.J. (2003b). Altered fatty acid composition of dopaminergic neurons expressing alphasynuclein and human brains with alpha-synucleinopathies. J Biol Chem 278, 49874-49881.

Shaw, P.J., Cirelli, C., Greenspan, R.J., and Tononi, G. (2000). Correlates of sleep and waking in Drosophila melanogaster. Science 287, 1834-1837.

Sheila M. Fleming, M.-F.C. (2009). Modeling Nonmotor Symptoms of Parkinson's Disease in Genetic Mouse Models. Advances in Behavioral Biology Volume 58

, 483-491.

Shirlee Tan, B.H., and Emiliana Borreli (2003). Dopaminergic mouse mutants: Investigating the roles of different Dopamine receptor subtypes and the Dopamine transporter. International Review of Neurobiology 54, 145-197.

Shults, C.W. (2006). Lewy bodies. Proc Natl Acad Sci U S A 103, 1661-1668.

Simon-Sanchez, J., Schulte, C., Bras, J.M., Sharma, M., Gibbs, J.R., Berg, D., Paisan-Ruiz, C., Lichtner, P., Scholz, S.W., Hernandez, D.G., et al. (2009). Genome-wide association study reveals genetic risk underlying Parkinson's disease. Nat Genet 41, 1308-1312.

Singleton, A.B., Farrer, M., Johnson, J., Singleton, A., Hague, S., Kachergus, J., Hulihan, M., Peuralinna, T., Dutra, A., Nussbaum, R., et al. (2003). alphaSynuclein locus triplication causes Parkinson's disease. Science 302, 841.

Smith, W.W., Margolis, R.L., Li, X., Troncoso, J.C., Lee, M.K., Dawson, V.L., Dawson, T.M., Iwatsubo, T., and Ross, C.A. (2005). Alpha-synuclein phosphorylation enhances eosinophilic cytoplasmic inclusion formation in SHSY5Y cells. J Neurosci 25, 5544-5552. 
Snyder, E.M., Nong, Y., Almeida, C.G., Paul, S., Moran, T., Choi, E.Y., Nairn, A.C., Salter, M.W., Lombroso, P.J., Gouras, G.K., et al. (2005). Regulation of NMDA receptor trafficking by amyloid-beta. Nat Neurosci 8, 1051-1058.

Soto, C. (2003). Unfolding the role of protein misfolding in neurodegenerative diseases. Nat Rev Neurosci 4, 49-60.

Spillantini, M.G., Schmidt, M.L., Lee, V.M., Trojanowski, J.Q., Jakes, R., and Goedert, M. (1997). Alpha-synuclein in Lewy bodies. Nature 388, 839-840.

Spradling, A.C., and Rubin, G.M. (1982). Transposition of cloned P elements into Drosophila germ line chromosomes. Science 218, 341-347.

Stewart, B.A., Atwood, H.L., Renger, J.J., Wang, J., and Wu, C.F. (1994). Improved stability of Drosophila larval neuromuscular preparations in haemolymph-like physiological solutions. J Comp Physiol A 175, 179-191.

Suh, J., and Jackson, F.R. (2007). Drosophila ebony activity is required in glia for the circadian regulation of locomotor activity. Neuron 55, 435-447.

Takamori, S., Holt, M., Stenius, K., Lemke, E.A., Gronborg, M., Riedel, D., Urlaub, H., Schenck, S., Brugger, B., Ringler, P., et al. (2006). Molecular anatomy of a trafficking organelle. Cell 127, 831-846.

Tompkins, M.M., and Hill, W.D. (1997). Contribution of somal Lewy bodies to neuronal death. Brain Res 775, 24-29.

Ueda, K., Fukushima, H., Masliah, E., Xia, Y., Iwai, A., Yoshimoto, M., Otero, D.A., Kondo, J., Ihara, Y., and Saitoh, T. (1993). Molecular cloning of cDNA encoding an unrecognized component of amyloid in Alzheimer disease. Proc Natl Acad Sci U S A 90, 11282-11286.

Ulmer, T.S., Bax, A., Cole, N.B., and Nussbaum, R.L. (2005). Structure and dynamics of micelle-bound human alpha-synuclein. J Biol Chem 280, 9595-9603.

Vilar, M., Chou, H.T., Luhrs, T., Maji, S.K., Riek-Loher, D., Verel, R., Manning, G., Stahlberg, H., and Riek, R. (2008). The fold of alpha-synuclein fibrils. Proc Natl Acad Sci U S A 105, 8637-8642.

Volles, M.J., and Lansbury, P.T., Jr. (2002). Vesicle permeabilization by protofibrillar alpha-synuclein is sensitive to Parkinson's disease-linked mutations and occurs by a pore-like mechanism. Biochemistry 41, 4595-4602. 
Volles, M.J., and Lansbury, P.T., Jr. (2007). Relationships between the sequence of alpha-synuclein and its membrane affinity, fibrillization propensity, and yeast toxicity. J Mol Biol 366, 1510-1522.

Walsh, D.M., Klyubin, I., Fadeeva, J.V., Cullen, W.K., Anwyl, R., Wolfe, M.S., Rowan, M.J., and Selkoe, D.J. (2002). Naturally secreted oligomers of amyloid beta protein potently inhibit hippocampal long-term potentiation in vivo. Nature $416,535-539$.

Weinreb, P. H., Zhen, W., Poon, A. W., Conway, K. A. and Lansbury, P. T., Jr. Biochemistry 1996, 35, 13709- 15

Whalley, K. (2007). Neurodegenerative disease: A good night's sleep

. Nature Reviews Neuroscience 8 .

White, B.H., Osterwalder, T.P., Yoon, K.S., Joiner, W.J., Whim, M.D., Kaczmarek, L.K., and Keshishian, H. (2001). Targeted attenuation of electrical activity in Drosophila using a genetically modified $\mathrm{K}(+)$ channel. Neuron 31, 699711.

Whitehead, D.L., Davies, A.D., Playfer, J.R., and Turnbull, C.J. (2008). Circadian rest-activity rhythm is altered in Parkinson's disease patients with hallucinations. Mov Disord 23, 1137-1145.

Wu, J.S., and Luo, L. (2006). A protocol for dissecting Drosophila melanogaster brains for live imaging or immunostaining. Nat Protoc 1, 2110-2115.

Yavich, L., Tanila, H., Vepsalainen, S., and Jakala, P. (2004). Role of alphasynuclein in presynaptic dopamine recruitment. J Neurosci 24, 11165-11170.

Ye, Y., Xi, W., Peng, Y., Wang, Y., and Guo, A. (2004). Long-term but not shortterm blockade of dopamine release in Drosophila impairs orientation during flight in a visual attention paradigm. Eur J Neurosci 20, 1001-1007.

Zhang, K., Guo, J.Z., Peng, Y., Xi, W., and Guo, A. (2007). Dopamine-mushroom body circuit regulates saliency-based decision-making in Drosophila. Science 316, 1901-1904.

Zimmerman, J.E., Naidoo, N., Raizen, D.M., and Pack, A.I. (2008). Conservation of sleep: insights from non-mammalian model systems. Trends Neurosci 31, 371376. 


\section{CURRICULUM VitAE}

\section{MADHU BABU GAJULA BALIJA}

$\begin{array}{ll}\text { First Name } & : \text { Madhu Babu } \\ \text { Last Name } & : \text { Gajula Balija } \\ \text { Date of Birth } & : 20^{\text {th }} \text { August1982 } \\ \text { Age } & : 27 \text { years } \\ \text { Gender } & : \text { Male } \\ \text { Nationality } & : \text { Indian } \\ \text { Languages Known } & : \text { English, Hindi, Telugu, Kannada, and basic German }\end{array}$

\section{PROFILE}

\section{Academic}

Strategic, independent and innovative thinker. Manifested leadership by proficiently seizing opportunities, establishing collaborations, setting direction and managing scientific projects. Mainly driven by curiosity, novelty, and impact. Worked with teams of diverse academic and cultural backgrounds. Specialized in Molecular Biology, Developmental Biology, and Genetics.

\section{Non-Academic}

Penchant for public service, demonstrated leadership and organizational skills, adept fund-raiser, and an ardent lover of liberal arts-business, history, politics and innovation. Firmly believes in social entrepreneurship and 'creative capitalism' for creating social impact.

\section{CAREER GOALS \& VISION}

\section{Short-term}

- To work towards international development, or to work at the interface of the corporate sector and government, or to work as a consultant in a multi-national company.

- To get admission into the MPP/MBA (Public Policy and Business Administration) program offered jointly by the Harvard Business School and Harvard Kennedy School of Government.

\section{Long-term}

- Aspire to deliver improved public services by influencing public policy, specifically for creating more opportunities in the developing countries by promoting innovation, research and development in science and technology. 


\section{EDUCATION}

- Ph.D. Molecular Biology (2006-Present)

International Max Planck Research School for Molecular Biology, Germany Max Planck Institute for Biophysical Chemistry, Goettingen, Germany

- M.Sc. Molecular Biology (2005-2006) International Max Planck Research School for Molecular Biology, Germany

- M.Sc. Biochemistry (2002-2004)

University of Hyderabad, Hyderabad, India

- B.Sc. Biochemistry, Chemistry, Zoology (1999-2002)

Osmania College, S.K. University, Anantapur, India

\section{SCHOLARSHIPS \& AWARDS}

- Internship at the WHO (World Health Organization) in Bonn, Germany (Oct - Dec, 2009)

- Stipend from the Max Planck Society, Germany (2006-Present)

- Stipend from the Swiss National Science Foundation, Switzerland (2005-2006)

- Stipend of the Excellence Foundation for the Promotion of the Max Planck Society, Germany (2004-2005)

- Merit Scholarship from the University of Hyderabad, India (2002-2004)

- Secured 2nd rank in the overall performance in M.Sc. Biochemistry at University of Hyderabad, India (2004)

- Summer Research Fellowship from the Indian Academy of Science, India (2003)

- Secured 4th rank in all India examination conducted by ICAR (Indian Council of Agricultural Research) for Junior Research Fellowships, India (2002)

\section{LEADERSHIP \& ORGANIZATIONAL SKILLS}

Motto: Making a difference by creating shared opportunities by building shared responsibilities through common interests and collective action.

- Founded the organization called SPARC (Society Promoters for Advancement, Redemption and Change) to bring awareness, to provide career guidance, and to encourage leadership among the youth of rural India (this project is still under the state development).

- Representative of PhD students (PhD Committee) at the Max Planck Institute for Biophysical Chemistry.

- Organized an international scientific conference Horizons in Molecular Biology 2008 and a career fair for scientists Horizons Career Fair 2008 with the support from the International Max Planck Research School for Molecular Biology.

- Organized career seminars and get-togethers/retreats (social and scientific) for the PhD students and Postdoctoral fellows at the Max Planck Institute for Biophysical Chemistry.

- Organized massive literacy campaigns in the small villages to spread the light of education as a member of the Students Union at University of Hyderabad (India).

- Fund-raising and Mediation: Raised nearly INR 3,00,000 (USD 6,200) to save 
the life of a patient suffering from the kidney failure due to Alport's Syndrome $\underline{\underline{\text { read }}}$ the story published in national daily The Hindu) by starting a donation drive (Navodayan Helpline) for his kidney transplantation and post-transplantation medical expenses.

Raised INR 40,000 (USD 850) for helping the tsunami victims in 2005.

Played an instrumental role in convincing our state's (Andhra Pradesh, India) medical ethics committee to allow banned kidney transplantation from a third party kidney donor in a special case.

\section{RESEARCH EXPERIENCE}

- Uncovering the role of pre-fibrillar oligomers of $\alpha$-synuclein in the pathogenesis of Parkinson's disease (2006-Present)

- Investigating the role of BRP85KDa (Bruchpilot) in peptidergic transmission

Prof. Dr. Herbert Jäckle, MPI for Biophysical Chemistry, Goettingen, Germany.

Prof. Dr. Christian Griesinger, MPI for Biophysical Chemistry, Goettingen, Germany.

Dr. Stefan Eimer, European Neuroscience Institute, Goettingen, Germany.

Prof. Dr. Stephan Sigrist, Freie University, Berlin, Germany.

- Generation of 'sev-wg' transgenic Drosophila for a genetic screen to identify new components of the Wnt signaling pathway

Developing an effective strategy to study cell-cell competition in mammals

Prof. Dr. Konrad Basler, Institute of Molecular Biology, University of Zürich, Zürich, Switzerland.

- Measuring the single exocytotic events by using patch-clamp technique

From 01-05-05 to 01-07-05 (Lab rotation 3)

Prof. Dr. Erwin Neher, MPI for Biophysical Chemistry, Goettingen, Germany.

- Elucidating the presence and role of maternally provided Dnd and Nanos in primordial germcell migration in Zebra fish embryos

From 01-03-2005 to 30-04-05 (Lab rotation 2)

Prof. Dr. Erez Raz, MPI for Biophysical Chemistry, Goettingen.

- Characterization of Upd (Drosophila JAK/STAT pathway ligand) secretion modifiers by $R N A i$

From 01-01-2005 to 28-02-05 (Lab rotation 1)

Prof. Dr. Martin Zeidler, MPI for Biophysical Chemistry, Goettingen.

- Elucidation of activities of lysosomal glycosidases from Xiphophorous fish embryonic cell line (A 2)

From 01-01-2004 to 30-04-2004.

Prof. Dr. N. Siva Kumar, Dept. of Biochemistry, University of Hyderabad, Hyderabad, India.

- Generation of point mutants of Eco.P15I.Mod (modification subunit of Eco.P15I type III restriction enzyme) by PCR based site directed mutagenesis

From 29-04-2003 to 30-07-2003 (Summer Research Fellow)

Prof. Dr. D. Narasimha Rao, Dept. of Biochemistry, Indian Institute of Science, Bangalore, India. 


\section{CONFERENCES}

2009

2008

2007

2007

2006 50th Annual Drosophila Research Conference, Chicago, IL, USA.

49th Annual Drosophila Research Conference, San Diego, CA, USA.

48th Annual Drosophila Research Conference, Philadelphia, PA, USA.

EMBO Conference on Chromatin and Epigenetics, Heidelberg, Germany.

International Conference on Magnetic Resonance in Biological Systems (ICMRBS), Goettingen, Germany.

\section{POSTER PRESENTATIONS}

Madhu Babu GB, Karpinar DP, Opazo F, Falkenburger B, Riedel D, Herzig A, Jaeckle H, Eimer S, Schulz JB, Griesinger C, Zweckstetter M.

Soluble pre-fibrillar oligomers of $\alpha$-synuclein in the neurodegeneration of Parkinson's disease. 49th Annual Drosophila Research Conference, San Diego, USA (2008).

Madhu Babu GB, Karpinar DP, Opazo F, Falkenburger B, Riedel D, Herzig A, Jaeckle H, Eimer S, Schulz JB, Griesinger C, Zweckstetter M.

Soluble pre-fibrillar oligomers of $\alpha$-synuclein in the neurodegeneration of Parkinson's disease. 50th Annual Drosophila Research Conference, Chicago, USA (2009).

\section{PUBLICATION}

Karpinar DP*, Balija $\mathrm{MB}^{*}$, Kügler $\mathrm{S}^{\star}$, Opazo $\mathrm{F}^{\star}$, Rezaei-Ghaleh N, Wender N, Kim HY, Taschenberger G, Falkenburger BH, Heise H, Kumar A, Riedel D, Fichtner L, Voigt A, Braus GH, Giller K, Becker S, Herzig A, Baldus M, Jäckle H, Eimer S, Schulz JB, Griesinger C, Zweckstetter M.

Pre-fibrillar alpha-synuclein variants with impaired beta-structure increase neurotoxicity in Parkinson's disease models. EMBO J. 28 (20): 3256-68, 2009.

*equal contribution of authors 
